\title{
Modern finite-size criticality: Dirichlet and Neumann boundary conditions
}

\author{
Messias V. S. Santos*, José B. da Silva Jr. ${ }^{\dagger}$, \\ Laboratório de Física Teórica e Computacional, Departamento de Física, \\ Universidade Federal de Pernambuco, 50670-901, Recife, PE, Brazil \\ Marcelo M. Leite $\ddagger$ \\ C N Yang Institute for Theoretical Physics, \\ State University of New York, Stony Brook, NY 11794-3840, USA
}

\begin{abstract}
Finite-size critical systems defined on a parallel plate geometry of finite extent along one single (z) direction with Dirichlet and Neumann boundary conditions at $z=0, L$ are analyzed in momentum space. We introduce a modified representation for the discrete eigenfunctions in a renormalized one-particle irreducible vertex part (1PI) scalar field-theoretic framework using either massless or massive fields. The appearance of multiplicities in the Feynman rules to construct diagrams due to this choice of representation of the basis functions is discussed along with the modified normalization conditions. For nonvanishing external quasi-momenta, Dirichlet and Neumann boundary conditions are shown to be unified within a single formalism. We examine the dimensional crossover regimes for these and show a correspondence with those from antiperiodic and periodic boundary conditions. It is demonstrated that finite-size effects for Dirichlet and Neumann boundary conditions do not require surface fields necessarily but are implemented nontrivially from the Feynman rules involving only bulk terms in the Lagrangian. As an application, the critical exponents $\eta$ and $\nu$ are evaluated at least up to two-loop level through diagrammatic means. We show that the critical indices are the same as those from the bulk (infinite) system irrespective of the boundary conditions.
\end{abstract}

PACS numbers: 64.60.an; 64.60.F-; 75.40.Cx

\footnotetext{
* e-mail:messiasvilbert@df.ufpe.br

$\dagger$ e-mail:jborba@petrobras.com.br

$\ddagger$ e-mail:mleite@insti.physics.sunysb.edu
} 


\section{INTRODUCTION}

Fields confined in a certain space region have especial properties whose study is worthwhile. Focusing entirely on conventional critical behavior, they correspond to the order parameters of certain systems undergoing a second order phase transition [1, 2]. The order parameter has well defined values at the boundary surfaces. This can be implemented through boundary conditions, e. g., periodic $(P B C)$, antiperiodic $(A B C)$, Dirichlet $(D B C)$, Neumann $(N B C)$, etc. and/or via external surface fields.

A layered parallel (hyper)plate geometry is defined by $(d-1)$-dimensional (hyper)planes of infinite extent whose limiting plates are located, say, at $z=0, L$ ( $z$ is the space direction perpendicular to the "planes"). We can either vary the distance between the boundaries or keep $L$ fixed and study universal amounts for the confined system. The change of fundamental quantities, like energy, with the variation on $L$ originates the Casimir effect [3-6], in which long range forces arise in response to the confinement of fluctuations. A similar effect in confined geometries of this type is the thinning of ${ }^{4} \mathrm{He}$ near the superfluid transition due to a force causing the change of the surface free energy in the critical fluctuations whenever the order parameter vanishes $(D B C)$ on both boundary surfaces [7].

By maintaining $L$ fixed, however, the critical behavior of the order parameter in thin films could manifest finite-size corrections. $P B C$ resembles closely experimental confined systems as in flows from glassy materials above and below the jamming transition in comparison with the unconfined behavior, whereas $D B C$ for the same system indicates a surface phenomenon [8]. Nevertheless, $D B C$ and $P B C$ take into account purely finite-size contributions in the experimental discussion of confined ${ }^{4} \mathrm{He}$ [9] as well as other fluids [10], between parallel plates. These corrections also take place in the measurements of spatial correlation of current critical points in an open billiards system [11] in describing the fields of turbulence, sound waves and acoustics, among others. On the other hand, Neumann boundary conditions govern the transition bulk-surface when the critical behavior deviates from the $(N, d)$ to the $(N, d-1)$ universality class and a nontrivial mixing of finite-size and surface effects was argued to take place [12]. Moreover, $D B C$ and $N B C$ have been investigated on a wide class of finite-size models, like amorphous solid suffering plastic deformation in a certain class of depinning models [13], dynamics of order reconstruction in confined nematic liquid crystals [14], determination of free energy corrections in the confined spherical model [15], etc. 
Phenomenological finite-size scaling theory states that close to the bulk critical temperature, the variable $\frac{L}{\xi}$ ( $\xi$ is the bulk correlation length) measures the deviation from the bulk critical behavior [16 20]. According to some authors [16 18], the description of the finite system is not limited to the values $\frac{L}{\xi}>1$ : they suggested that the finite-size critical exponents should be identical as those from the bulk (infinite) system.

The description of finite-size critical systems using momentum space renormalization group field-theoretic methods has basic aspects which are simple to grasp. First, for parallel plate geometries the boundary conditions on the plates are implemented through the bare free propagator. Second, the typical length $L$ separating the boundaries can be included in the Feynman rules. For $P B C$ and $A B C$, the momentum along the finite-size direction turns into the quasi-momentum and the integral along the $z$ direction gets transformed to an infinite sum (Nemirovsky-Freed $(N F)$ method [21]). Motivated by the $N F$ Green's functions formalism, a one-particle irreducible $(1 P I)$ vertex part framework was designed recently to the treatment of finite-size systems subject to $P B C$ and $A B C$ using either massive or massless fields. Within this finite-size technique, scaling theory holds rigorously in the whole region $0<\frac{L}{\xi} \leq \infty$ in agreement with previous claims [18, 20]. Explicit computations at higher order were performed corroborating that the finiteness corrections are not sufficient to modify the bulk critical exponents [22]. A different piece of folklore exists in the literature regarding $D B C$ and $N B C$ : they represent free surfaces and are appropriate to describe finite-size plus surface effects. Indeed one can introduce surface fields in conjumination with bulk fields for this boundary conditions breaking translation invariance in this way [23]. If we allow only bulk fields, ruling out ordering surface fields associated with boundary plates, is that possible to renormalize solely bulk fields subject to those boundary conditions which do not produce any surface contributions, but with manifest translation invariance breaking? Can a framework for $D B C$ and $N B C$ be devised to explore the full finite-size region $0<\frac{L}{\xi} \leq \infty$ ? If we achieve consistency with the results obtained from $P B C$ and $A B C$, the emerging description represents a modern finite-size scaling regime. Is there any simple relation with the associated unconfined system (the "bulk" criticality)? How the decreasing of the confinement region rules over those situations? How the presence or absence of surface fields alters the criticality?

In this work we build up an ab initio renormalized one-particle irreducible vertex part formalism in momentum space for Dirichlet and Neumann boundary conditions in order 
to calculate perturbatively universal critical properties including only bulk fields. For sake of comparison with previous one-loop results, we first employ massive fields. We modify the Feynman rules with respect to the previous approach using sines and cosines as basis functions [21] due to our decomposition of them in terms of exponentials only. The Feynman diagrams obtained from those rules can be expressed in terms of integrals (and summations) identical to the $P B C$ and $A B C$ cases, but contain extra "nondiagonal" terms in which there are not as many summations as there are integrals over the $(d-1)$-dimensional transverse space.

This new feature will permit us to prove that each diagram is composed of two parts: the first one, where the momenta as well as quasimomenta are conserved in every diagram order by order in perturbation theory and the second, substantiated by the presence of the "nondiagonal" terms which break the translation invariance (violation of quasi-momentum conservation) along the finite-size direction. These terms are important for they make a clear-cut distinction in comparison with the simplicity obtained from $P B C$ and $A B C$ results. They look like surface contributions at first sight albeit they are purely finite-size corrections and perhaps this is the mathematical origin of the folklore above mentioned. We will show that they do not contribute to the leading singularities in dimensional regularization. We introduce new Feynman rules for vertex parts including composite operators which can be renormalized multiplicatively. We demonstrate that for a certain quasi-momentum distribution of external legs not belonging to composite fields, the external quasi-momentum of the insertion of the composite operator admits more than one combination of the external quasi-momenta of the other usual external legs (not associated to composite operators) and should be properly taking into account in the set of rules order by order in perturbation theory.

We discuss the dimensional crossover criteria and show the consistency with the previous results from $P B C$ and $A B C$ in [22]. Staying away from the problematic region where the $\epsilon$-expansion ceases to give meaningful results, all loop integrals considered will be shown to have the general structure made out of bulk plus finite-size terms with the latter depending on the boundary conditions. Within the finite-size plus bulk regime, we choose to renormalize the field theory with zero external momenta and nonvanishing external quasimomenta in order to unify Neumann and Dirichlet boundary conditions in a single framework. Afterwards, the unifying formalism of massless fields using nonvanishing external momenta and 
quasi-momenta for $D B C$ and $N B C$ is presented along with a discussion of the dimensional crossover criterion where the finite-size regime starts to give meaningless results. We prove its equivalence with the massive case. As an application we compute the critical exponents $\eta$ and $\nu$ using diagrammatic means. We show that the universal results are independent of the boundary condition.

In Sec. II we discuss how the discrete eigenfunctions corresponding to $N B C$ and $D B C$ are expressed in terms of exponentials and the consequent modification of the Feynman rules. We construct the tensor couplings for all the primitively divergent vertex parts which can be renormalized multiplicatively and demonstrate the emergence of multiplicities with respect to the bulk (infinite) theory due to the exponential representation of the basis functions. We show that $D B C$ possess diagram multiplicities identical to $N B C$ whenever both theories are defined at nonvanishing external quasi-momenta. In this picture we compute explicitly some sample Feynman diagrams. We conclude by presenting the unified set of all diagrams which are going to be relevant in our discussion. The arguments are valid for both formulations of massive and massless fields.

We discuss the renormalization of massive fields in Sec. III. We start with a large number of diagrams and achieve the reduction to a smaller set of graphs owing to the nontrivial cancellation of the mass insertions. This feature includes both the diagonal and nondiagonal terms of tadpole diagrams. This argument is decisive in proving that the theory can be renormalized without making any reference to ad hoc surface fields. Normalization conditions are defined for the primitively divergent vertex parts in order to assure the finiteness of all vertex parts that can be renormalized multiplicatively. We briefly discuss how the flow in the mass scale affects the renormalized vertex parts in essentially the same way as in the bulk case. The limit $L \rightarrow \infty$ is shown to retrieve the bulk result, whereas the $L \rightarrow 0$ limit marks the onset of the dimensional crossover which invalidates $\epsilon$-expansion results. We also list the solution for the higher loop diagrams from Appendix A which will be required in the determination of critical exponents in Sec. IV. The dependence of the renormalization constants/functions on the boundary conditions disappears in the final expression for the exponents.

Section V contains the explicit discussion of massless fields and their multiplicative renormalization for both boundary conditions in the unified description. The examination of how mass insertions are cancelled is explained in two different ways in order to get a minimal 
number of diagrams to work with in the determination of critical exponents. An in-depth discussion on the validity of the finite-size regime with the approach to the bulk criticality as well as to the dimensional crossover regime is presented. We give a brief description of the solution to the higher-order massless diagrams by writing down their expressions and point out their similarity with $P B C$ and $A B C$ arguments for the massless fields. In Sec. VI we compute the critical exponents in the massless approach.

In Sec. VII we discuss our results. The dimensional crossover regime is analyzed explicitly by focusing on the one-loop correction to the bulk case of the four-point vertex part. We perform a comparison of these regimes with those from $P B C$ and $A B C$ previously studied. We vary the correlation length and establish that even in the massive theory there are regions for fixed, finite $\xi$ where $\frac{L}{\xi}<1$ and the $\epsilon$-expansion results are still valid.

Section VIII displays the conclusions and future directions within the formalism introduced in the present work. In addition, we point out how these new aspects can be adapted to tackle the problem of competing systems.

In Appendix $\mathrm{A}$ we compute higher order massive integrals. We decided not to give a detailed account of the solution of massless integrals of this problem in another appendix for the same reason. The reader is advised to consult Appendix A and Ref. [22] for grasping the details.

\section{MODIFIED $N F$ APPROACH TO FEYNMAN RULES WITH $D B C$ AND $N B C$}

\section{A. Review of $N F$ approach}

First we will discuss briefly the field theory setting introduced by Nemirovsky and Freed $(N F)$ [21] for constructing Feynman diagrams in momentum space with the boundary conditions of interest in the present work. We are going to restrict ourselves to the situation where no external surface fields are allowed.

The bare Lagrangian (free energy) density is composed by scalar fields with $O(N)$ symmetry, defined on the volume enclosed by the two limiting $(d-1)$-dimensional parallel hyperplates located at $z=0, L$ (bulk fields). It is given by:

$$
\mathcal{L}=\frac{1}{2}\left|\nabla \phi_{0}\right|^{2}+\frac{1}{2} \mu_{0}^{2} \phi_{0}^{2}+\frac{1}{4 !} \lambda_{0}\left(\phi_{0}^{2}\right)^{2}
$$

where $\phi_{0}, \mu_{0}$ and $\lambda_{0}$ are the bare order parameter, mass $\left(\mu_{0}^{2}=t_{0}\right.$ is the bare reduced 
temperature proportional to $\frac{T-T_{C}}{T_{C}}$ ) and coupling constant, respectively [2, 27, 28]. The $O(N)$ symmetry of the bare order parameter means that it is a vector of $N$ components $\left(\left(\phi_{0}^{2}\right)^{2}=\left(\phi_{01}^{2}+\ldots+\phi_{0 N}^{2}\right)^{2}\right)$. The additional indices related to the $O(N)$ symmetry of the fields were not written since they are identical to the infinite system. The space directions split in two distinct sets denoted by $\vec{\rho}$ representing the coordinates along the $(d-1)$-dimensional subspace parallel to the plates and the perpendicular $z$ axis, collectively represented in the vector form as $x=(\vec{\rho}, z)$. The space is layered and filled with parallel plates in the region between $z=0$ and $z=L$. The field satisfies $\phi_{0}(z=0)=\phi_{0}(z=L)=0$ for Dirichlet boundary conditions $(D B C)$, whereas $\frac{\partial \phi_{0}}{\partial z}(z=0)=\frac{\partial \phi_{0}}{\partial z}(z=L)$ for Neumann boundary conditions $(N B C)$.

The order parameter can be related to its Fourier modes in momentum space through $\phi_{0}(x)=\sum_{j} \int d^{d-1} k \exp (i \vec{k} \cdot \vec{\rho}) u_{j}(z) \phi_{0 j}(\vec{k})$, where $\vec{k}$ is the momentum vector characterizing the $(d-1)$-dimensional space. The basis functions $u_{j}(z)$ satisfy the differential equation $-\frac{d^{2} u_{j}(z)}{d z^{2}}=\kappa_{j}^{2} u_{j}(z), \kappa_{j}$ being the quasi-momentum along the $z$-direction. The eigenfunctions are orthonormalized according to $\sum_{j} u_{j}(z) u_{j}\left(z^{\prime}\right)=\delta\left(z-z^{\prime}\right)$ and $\int_{0}^{L} d z u_{j}(z) u_{j^{\prime}}^{*}(z)=\delta_{j, j^{\prime}}$. Here $\kappa_{j}=\frac{\pi j}{L} \equiv \tilde{\sigma} j$, where $j=1,2, \ldots$ for $D B C$ and $j=0,1,2, \ldots$, for $N B C\left(\tilde{\sigma}=\frac{\pi}{L}\right)$. We can attach a label for each boundary condition such that $\tau=-1$ corresponds to DBC and $\tau=1$ to NBC. The free bare massive propagator $\left(\mu_{0}^{2} \neq 0\right)$ in momentum space for either boundary condition is given by the expression $G_{0 j}^{(\tau)}(k, j)=\frac{1}{k^{2}+\tilde{\sigma}^{2} j^{2}+\mu_{0}^{2}}$. Although not explicitly mentioned so far, it is obvious that the eigenfunctions depend upon the boundary conditions. We will suppress their relation with the boundary conditions but can retrieve this dependence whenever it is appropriate.

A generic Feynman diagram involves the product of many bare propagators $G_{0 j}^{(\tau)}$ contracted with interaction vertices. The amazing fact about the structure of the finitesize to take effect on the field theory is that it is implemented as an internal symmetry. For example, beside the standard tensorial couplings of the infinite theory corresponding to the $N$-component order parameter, each momentum line (propagator) must be multiplied by $S_{j_{1} j_{2}}^{(\tau)}=\int_{0}^{L} d z u_{j_{1}}(z) u_{j_{2}}(z)$ and the $\phi^{4}$ vertices are multipled by the tensor $S_{j_{1} j_{2} j_{3} j_{4}}^{(\tau)}=\int_{0}^{L} d z u_{j_{1}}(z) u_{j_{2}}(z) u_{j_{3}}(z) u_{j_{4}}(z)$. Forgetting about the composite operators for the time being, this is nothing but the enhanced internal symmetry representation of the direct product $O(N) \times(f s)$, where the $(f s)$ symmetry is represented by the tensors $S_{j_{1} j_{2}}^{(\tau)}$ and 
$S_{j_{1} j_{2} j_{3} j_{4}}^{(\tau)}$. The basis functions can be written as $u_{j}^{(\tau=-1)}(z)=\left(\frac{2}{L}\right)^{\frac{1}{2}} \sin \left(\kappa_{j} z\right)$ for $D B C$, whereas for $N B C$ we have the nonzero mode as in $D B C u_{j}^{(\tau=1)}(z)=\left(\frac{2}{L}\right)^{\frac{1}{2}} \cos \left(\kappa_{j} z\right)(j=1,2 \ldots)$ as well as $u_{0}^{(\tau=1)}=\left(\frac{1}{L}\right)^{\frac{1}{2}}$. The Feynman rules for vertex and propagators are given in [21].

In the computation of Feynman diagrams as the set of integrals in $(d-1)$ dimensions in conjunction with infinite summations, it is difficult to establish a direct comparison with periodic $(P B C)$ and antiperiodic boundary conditions $(A B C)$. The reason is that $j \geq 0$ for $D B C$ and $N B C$, but varies in the interval $(-\infty, \infty)$ por $P B C$ and $A B C$. The simplification which took place for $P B C$ and $A B C$ when we could compute integrals with all external quasi-momentum set to zero no longer occurs for $D B C$. In any serious attempt to unify the framework for $D B C$ and $P B C$, we should figure out how to compute graphs with nonvanishing external quasi-momentum for those conditions.

Fortunately, at an external quasi-momentum symmetric point, we can compute the diagrams provided some modifications are introduced in the moding of the label $j$ and additional trivial orthonormality properties. Next, we shall introduce new notation in order to give a unified description of the Dirichlet and Neumann problems for nonzero external quasi-momentum.

\section{B. Exponential representation and unification of the Feynman rules for nonvan-} ishing external quasi-momentum

\section{One-loop diagrams for the two- and four-point vertex functions}

The construction of Feynman diagrams for $D B C$ (sine) and $N B C$ (cosine) takes into account solely the internal structure provided by the tensors $S_{j_{1} j_{2}}^{(\tau)}, S_{j_{1} j_{2} j_{3} j_{4}}^{(\tau)}$. The $O(N)$ underlying symmetry appears in exactly the same form as in the infinite $L$ limit and we shall simply attach the symmetry factor to each diagram under consideration. However, we are going to discuss explicitly how the combination of products of the finite size tensors shows up in particular one-loop graphs. We wish to express them in terms of summations varying in the range $(-\infty, \infty)$ and $(d-1)$-dimensional momentum integrals.

Let us write the basis functions as

$$
u_{j}^{(\tau=-1)}(z)=\left(\frac{2}{L}\right)^{\frac{1}{2}}\left[\frac{1}{2 i}\left(e^{i \tilde{\sigma} j z}-e^{-i \tilde{\sigma} j z}\right)\right] \quad(D B C),
$$




$$
u_{j}^{(\tau=1)}(z)=\left(\frac{2}{L}\right)^{\frac{1}{2}}\left[\frac{1}{2}\left(e^{i \tilde{\sigma} j z}+e^{-i \tilde{\sigma} j z}\right)\right] \quad(N B C) .
$$

We can compute the tensors in this new representation. For instance, using the definition of $S_{j_{1} j_{2}}^{(\tau)}$ and restricting ourselves only to positive values $\left(j_{1}, j_{2} \in Z_{+}^{*}\right)$ we achieve the following unified form

$$
S_{j_{1} j_{2}}^{(\tau)}=\frac{(-1)^{\frac{1-\tau}{2}}}{2 L} \int_{0}^{L} d z\left[e^{i \tilde{\sigma}\left(j_{1}+j_{2}\right) z}+e^{-i \tilde{\sigma}\left(j_{1}+j_{2}\right) z}+\tau e^{i \tilde{\sigma}\left(j_{1}-j_{2}\right) z}+\tau e^{-i \tilde{\sigma}\left(j_{1}-j_{2}\right) z}\right],
$$

which by the change $z \rightarrow-z$ in the second and fourth terms turns out to be given by:

$$
S_{j_{1} j_{2}}^{(\tau)}=(-1)^{\frac{1-\tau}{2}}\left[\tau \delta_{j_{1}-j_{2}, 0}+\delta_{j_{1}+j_{2}, 0}\right] \equiv(-1)^{\frac{1-\tau}{2}}\left[\tau \delta\left(j_{1}-j_{2}\right)+\delta\left(j_{1}+j_{2}\right)\right]
$$

The new notation for the Kronecker's delta is going to be very useful in what follows. We have to be careful with the moding since $N B C$ allows $j_{1}, j_{2}=0$ in the construction of a generic Feynman diagram: in the contraction of these tensors, this value of $j$ can occur and all components of the finite-size tensors should be computed (as the internal quasimomentum indices are summed). For all practical purposes in the present paper, the result for the two-index tensor is simply $S_{j_{1} j_{2}}^{(\tau)}=\delta\left(j_{1}-j_{2}\right)$.

It is a simple task to evaluate the tensor $S_{j_{1} j_{2} j_{3} j_{4}}^{(\tau)}$ with the arguments at hand. We find

$$
\begin{aligned}
S_{j_{1} j_{2} j_{3} j_{4}}^{(\tau)=\frac{1}{2 L}}[ & \delta\left(j_{1}+j_{2}+j_{3}+j_{4}\right)+\tau \delta\left(j_{1}-j_{2}+j_{3}+j_{4}\right)+\tau \delta\left(j_{1}+j_{2}-j_{3}+j_{4}\right) \\
& +\tau \delta\left(j_{1}+j_{2}+j_{3}-j_{4}\right)+\delta\left(j_{1}-j_{2}-j_{3}+j_{4}\right)+\delta\left(j_{1}-j_{2}+j_{3}-j_{4}\right) \\
& \left.+\delta\left(j_{1}+j_{2}-j_{3}-j_{4}\right)+\tau \delta\left(j_{1}-j_{2}-j_{3}-j_{4}\right)\right] .
\end{aligned}
$$

In $P B C$ and $A B C$ the quasi-momentum conservation is represented by the condition $S_{j_{1} j_{2} j_{3} j_{4}}^{(\tau)}=\frac{1}{L} \delta\left(j_{1}+j_{2}+j_{3}+j_{4}\right)$, whereas $D B C$ and $N B C$ in the above expression possess the quasi-momentum conservation as well as additional contributions as expected from the intrinsic nontrivial nature of those boundary conditions.

The last expression can be further simplified utilizing the notation $\tilde{j}_{ \pm}=j_{1} \pm j_{2}, j_{ \pm}=j_{3} \pm j_{4}$ which yields

$$
S_{j_{1} j_{2} j_{3} j_{4}}^{(\tau)}=\frac{1}{2 L} \sum_{\substack{\alpha_{1}= \pm 1 \\ \alpha_{2}= \pm 1 \\ \beta= \pm 1}}\left(\alpha_{1} \alpha_{2}\right)^{\frac{1-\tau}{2}} \delta\left(\tilde{j}_{\alpha_{1}}+\beta j_{\alpha_{2}}\right)
$$

Let us consider the components of the finite-size tensors when $j=0$. In the simplest case of the two-index tensor, it is easy to show that zero modes do not mix with nonzero modes, 
which can be expressed in a compact form as $S_{0 j}^{(\tau)}=\delta(j) \delta^{\tau 1}$. When we analyze the four-index tensor, the only difference with respect to the components with all nonvanishing subscripts is different normalization factors. It is straightforward to prove that $S_{0 j_{1} j_{2} j_{3}}^{(\tau)}=\frac{1}{\sqrt{2} L}\left[\delta\left(j_{1}+j_{2}+\right.\right.$ $\left.\left.j_{3}\right)+\delta\left(j_{1}-j_{2}+j_{3}\right)+\delta\left(j_{1}+j_{2}-j_{3}\right)+\delta\left(j_{1}-j_{2}-j_{3}\right)\right] \delta^{\tau 1}, S_{00 j_{1} j_{2}}^{(\tau)}=\frac{1}{L}\left[\delta\left(j_{1}+j_{2}\right)+\delta\left(j_{1}-j_{2}\right)\right] \delta^{\tau 1}$ and $S_{000 j_{1}}^{(\tau)}=\frac{1}{L} \delta\left(j_{1}\right) \delta^{\tau 1}$. It is important to mention that neither $S_{00 j_{1} j_{2}}^{(\tau)}$ nor $S_{0 j_{1} j_{2} j_{3}}^{(\tau)}$ are equivalent to $N F$ notation. The above tensor is similar but has different components when compared with the NF counterpart (obtained in terms of sines and cosines) as can be verified in Eq. (A6) from the second paper in Ref. [21]. Therefore, the components of the four-index tensor in the exponential representation enlarges the possibilities for the finite-size indices.

These elements will suffice to our construction of vertex parts diagrams not including composite fields yet. Before working out explicitly the one-loop graphs, let us introduce the notation $\tilde{S}_{j_{1} j_{2} j_{3} j_{4}}^{(\tau)}=2 \pi S_{j_{1} j_{2} j_{3} j_{4}}^{(\tau)}$ and from now on we are going to construct the integrals associated with Feynman diagrams with this modified tensor. Each loop integral will be represented by a $(d-1)$-dimensional integral multiplied by summations involving products of $S_{j_{1} j_{2}}^{(\tau)}$ and $\tilde{S}_{j_{1} j_{2} j_{3} j_{4}}^{(\tau)}$. This has the virtue of producing the metric factor $\tilde{\sigma}$ multiplying each loop integral.

In practice, we have to use the tensor structure to build up the diagrams perturbatively. The coupling constant factors will be omitted in all diagrams to be discussed, but will be retrieved during the discussion of the diagrammatic expansion of each primitively divergent vertex part. Within a $1 P I$ vertex part framework, those primitively divergent are the two-, four- and two-point with one composite operator vertex parts, which are represented by $\Gamma^{(2)}, \Gamma^{(4)}$ and $\Gamma^{(2,1)}$, respectively. Whenever we do not mention explicitly the external quasi-momentum index in a certain graph, these modes are arbitrary. For instance, a generic graph from $\Gamma^{(2)}$ has external quasi-momenta labeled by $i_{1}$ and $i_{2}, \Gamma^{(4)}$ has associated external quasi-momenta $i_{1}, i_{2}, i_{3}$ and $i_{4}$ and so on. On the other hand, we are going to attach them to the particular diagram whenever we choose a particular fixed distribution of external quasi-momenta modes.

As a first sample computation, consider the one-loop "tadpole" contribution to $\Gamma^{(2)}$. Its diagrammatic expression reads

$$
\bigcirc=\frac{(N+2)}{3} \sum_{l_{1}, l_{2} \geq 0}^{\infty} \tilde{S}_{i_{1} i_{2} l_{1} l_{2}}^{(\tau)} S_{l_{1} l_{2}}^{(\tau)} \int d^{d-1} q G_{0}\left(q, l_{1}\right) .
$$

Let us consider explicitly Neumann boundary conditions $(\tau=1)$. The summation splits in 
the contributions of zero and nonzero modes as:

$$
\begin{aligned}
& \sum_{l_{1}, l_{2} \geq 0}^{\infty} \tilde{S}_{i_{1} i_{2} l_{1} l_{2}}^{(\tau)} S_{l_{1} l_{2}}^{(\tau)} \int d^{d-1} k G_{0}\left(q, l_{1}\right)=\tilde{S}_{i_{1} i_{2} 00}^{(1)} S_{00}^{(1)} \int d^{d-1} k G_{0}(q, 0)+2 \sum_{l=1}^{\infty} \tilde{S}_{i_{1} i_{2} l 0}^{(1)} S_{l 0}^{(1)} \\
& \int d^{d-1} k G_{0}(q, l)+\sum_{l_{1}, l_{2}=1}^{\infty} \tilde{S}_{i_{1} i_{2} l_{1} l_{2}}^{(1)} S_{l_{1} l_{2}}^{(1)} \int d^{d-1} k G_{0}\left(q, l_{1}\right) .
\end{aligned}
$$

Note that $G_{0}(q, l)=G_{0}(q,-l)$. The term proportional to $S_{l 0}^{(1)}$ vanishes. Replacing explicitly the values of the tensors already derived and defining $i_{ \pm}=i_{1} \pm i_{2}$, we find:

$$
\begin{aligned}
& \sum_{l_{1}, l_{2} \geq 0}^{\infty} \tilde{S}_{i_{1} i_{2} l_{1} l_{2}}^{(\tau)} S_{l_{1} l_{2}}^{(\tau)} \int d^{d-1} k G_{0}\left(q, l_{1}\right)=\tilde{\sigma} \int d^{d-1} k\left[2\left(\delta\left(i_{+}\right)+\delta\left(i_{-}\right)\right) G_{0}(q, 0)+\sum_{l=1}^{\infty}\left(\delta\left(i_{+}+2 l\right)\right.\right. \\
& \left.\left.+\delta\left(i_{+}-2 l\right)+2 \delta\left(i_{+}\right)+\delta\left(i_{-}+2 l\right)+\delta\left(i_{-}-2 l\right)+2 \delta\left(i_{-}\right)\right) G_{0}(q, l)\right] .
\end{aligned}
$$

Next, the identities

$$
\begin{aligned}
& \sum_{l \neq 0, l=-\infty}^{\infty} \delta\left(i_{ \pm}+2 l\right) G_{0}(q, l)=\sum_{l=-\infty}^{\infty} \delta\left(i_{ \pm}+2 l\right) G_{0}(q, l)-\delta\left(i_{ \pm}\right) G_{0}(q, 0), \\
& \delta\left(i_{ \pm}\right) G(q, 0)+2 \delta\left(i_{ \pm}\right) \sum_{l=1}^{\infty} G_{0}(q, l)=\delta\left(i_{ \pm}\right) \sum_{l=-\infty}^{\infty} G_{0}(q, l)
\end{aligned}
$$

will be useful in order to achieve our goal of transforming summations with a bounded lower limit into those with unlimited negative values for the index $l$. After some manipulations the final expression can be rewritten as

$$
\bigcirc=\tilde{\sigma} \frac{(N+2)}{3} \int d^{d-1} q \sum_{l=-\infty}^{\infty} \frac{\left[\delta\left(i_{-}\right)+\delta\left(i_{+}\right)+\delta\left(i_{+}+2 l\right)+\delta\left(i_{-}-2 l\right)\right]}{q^{2}+\tilde{\sigma}^{2} l^{2}+\mu_{0}^{2}} .
$$

For $D B C$ boundary conditions we have instead

$$
\bigcirc=\tilde{\sigma} \frac{(N+2)}{3} \sum_{l_{1}, l_{2}=1}^{\infty} \tilde{S}_{i_{1} i_{2} l_{1} l_{2}}^{(-1)} S_{l_{1} l_{2}}^{(-1)} \int d^{d-1} k G_{0}\left(k, l_{1}\right) .
$$

With $l_{1}, l_{2}>0, S_{l_{1}, l_{2}}^{(-1)}=\delta\left(l_{1}-l_{2}\right)$. We can perform one summation, say over $l_{2}$ and perform the change of index $l_{1} \rightarrow l$ in the remaining summation. Focusing only on the summation and replacing the expression for $\tilde{S}_{i_{1} i_{2} l l}^{(-1)}$ we get to

$$
\begin{aligned}
& \sum_{l=1}^{\infty} \tilde{S}_{i_{1} i_{2} l l}^{(-1)} \int d^{d-1} k G_{0}(k, l)=\tilde{\sigma} \sum_{l=1}^{\infty}\left[-2 \delta\left(i_{+}\right)+\delta\left(i_{+}+2 l\right)+\delta\left(i_{+}-2 l\right)+2 \delta\left(i_{-}\right)-\delta\left(i_{-}+2 l\right)\right. \\
& \left.-\delta\left(i_{-}-2 l\right)\right] \int d^{d-1} k G_{0}(k, l) .
\end{aligned}
$$


From the identity $\sum_{l=1}^{\infty}\left(\delta\left(i_{ \pm}+2 l\right)+\delta\left(i_{ \pm}-2 l\right)\right) G_{0}(q, l)=\sum_{l=-\infty}^{\infty} \delta\left(i_{ \pm}+2 l\right) G_{0}(q, l)$ and by employing Eqs. (11), we obtain

$$
\bigcirc=\tilde{\sigma} \frac{(N+2)}{3} \int d^{d-1} q \sum_{l=-\infty}^{\infty} \frac{\left[\delta\left(i_{-}\right)-\delta\left(i_{+}\right)+\delta\left(i_{+}+2 l\right)-\delta\left(i_{-}-2 l\right)\right]}{q^{2}+\tilde{\sigma}^{2} l^{2}+\mu_{0}^{2}} .
$$

Comparing the $D B C(\tau=-1)$ and $N B C(\tau=1)$ results, we can write down the expression corresponding to this graph in a unified fashion for these boundary conditions as

$$
\bigcirc=\tilde{\sigma} \frac{(N+2)}{3} \int d^{d-1} q \sum_{l=-\infty}^{\infty} \frac{\left[\delta\left(i_{-}\right)+\tau \delta\left(i_{+}\right)+\delta\left(i_{+}+2 l\right)+\tau \delta\left(i_{-}-2 l\right)\right]}{q^{2}+\tilde{\sigma}^{2} l^{2}+\mu_{0}^{2}} .
$$

The summations in the third and fourth term can be performed and we are left with

$$
\bigcirc=\frac{(N+2)}{3}\left[\left(\delta\left(i_{-}\right)+\tau \delta\left(i_{+}\right)\right) D_{1}\left(\tilde{\sigma}, \mu_{0}\right)+\tau \tilde{D}_{1}\left(\frac{i_{-}}{2}, \tilde{\sigma}, \mu_{0}\right)+\tilde{D}_{1}\left(\frac{i_{+}}{2}, \tilde{\sigma}, \mu_{0}\right)\right]
$$

where

$$
\begin{aligned}
& D_{1}\left(\tilde{\sigma}, \mu_{0}\right)=\tilde{\sigma} \sum_{l=-\infty}^{\infty} \int \frac{d^{d-1} q}{q^{2}+\tilde{\sigma}^{2} l^{2}+\mu_{0}^{2}} \\
& \tilde{D}_{1}\left(i, \tilde{\sigma}, \mu_{0}\right)=\tilde{\sigma} \int \frac{d^{d-1} q}{q^{2}+\tilde{\sigma}^{2} i^{2}+\mu_{0}^{2}}
\end{aligned}
$$

Although the first and second terms in the diagram expression have a clear counterpart in $P B C$ and $A B C$, the third and fourth terms are entirely new, since no summation appears there. They look like surface terms albeit coming purely from finite-size contributions: they reflect the breaking of translation invariance for $D B C$ and $N B C$ along the finite-size direction. In what follows diagrams of this type ("tadpoles") will not be required in the present method as we are going to show in the discussion of normalization conditions.

Consider the one-loop contribution of the four-point vertex part $\Gamma^{(4)}$. Its graph in the present setting is given by

$$
\chi=\frac{(N+8)}{9} \sum_{l_{1}, l_{2} \geq 0}^{\infty} \tilde{S}_{i_{1} i_{2} l_{1} l_{2}}^{(\tau)} \tilde{S}_{l_{1} l_{2} i_{3} i_{4}}^{(\tau)} \int d^{d-1} q G_{0}\left(q+k, l_{1}\right) G_{0}\left(q, l_{2}\right),
$$

where $k$ is the external momentum, $i_{1}, i_{2}, i_{3}$ and $i_{4}$ are the external indices from quasimomenta. We start with $\tau=1(N B C)$. Focusing only on the summmation, integrals and 
propagator products, we first decompose the whole thing in the following form

$$
\begin{aligned}
& \sum_{l_{1}, l_{2} \geq 0}^{\infty} \tilde{S}_{i_{1} i_{2} l_{1} l_{2}}^{(\tau)} \tilde{S}_{l_{1} l_{2} i_{3} i_{4}}^{(\tau)} \int d^{d-1} q G_{0}\left(q+k, l_{1}\right) G_{0}\left(q, l_{2}\right)=\int d^{d-1} q\left[\tilde{S}_{i_{1} i_{2} 00}^{(1)} \tilde{S}_{00 i_{3} i_{4}}^{(1)} G_{0}(q+k, 0)\right. \\
& G_{0}(q, 0)+\sum_{l_{1}=1}^{\infty} \tilde{S}_{i_{1} i_{2} l_{1} 0}^{(\tau)} \tilde{S}_{l_{1} 0 i_{3} i_{4}}^{(\tau)} G_{0}\left(q+k, l_{1}\right) G_{0}(q, 0)+\sum_{l_{2}=1}^{\infty} \tilde{S}_{i_{1} i_{2} 0 l_{2}}^{(\tau)} \tilde{S}_{0 l_{2} i_{3} i_{4}}^{(\tau)} G_{0}(q+k, 0) G_{0}\left(q, l_{2}\right) \\
& \left.+\sum_{l_{1}, l_{2}=1}^{\infty} \tilde{S}_{i_{1} i_{2} l_{1} l_{2}}^{(\tau)} \tilde{S}_{l_{1} l_{2} i_{3} i_{4}}^{(\tau)} G_{0}\left(q+k, l_{1}\right) G_{0}\left(q, l_{2}\right)\right] .
\end{aligned}
$$

Note that the single summations are actually equal. For the sake of simplicity, the definitions $i_{ \pm}=i_{1} \pm i_{2}$ and $\tilde{i}_{ \pm}=i_{3} \pm i_{4}$ will have their utility in what follows. Using the expressions for the tensors and the symmetry of the integrals yield

$$
\begin{aligned}
& \sum_{l_{1}, l_{2} \geq 0}^{\infty} \tilde{S}_{i_{1} i_{2} l_{1} l_{2}}^{(\tau)} \tilde{S}_{l_{1} l_{2} i_{3} i_{4}}^{(\tau)} \int d^{d-1} q G_{0}\left(q+k, l_{1}\right) G_{0}\left(q, l_{2}\right)=4 \tilde{\sigma}^{2} \int d^{d-1} q\left([ \delta ( i _ { + } ) + \delta ( i _ { - } ) ] \left[\delta\left(\tilde{i}_{+}\right)\right.\right. \\
& \left.+\delta\left(\tilde{i}_{-}\right)\right] G_{0}(q+k, 0) G_{0}(q, 0)+\sum_{l=1}^{\infty}\left[\delta\left(i_{+}+l\right)+\delta\left(i_{+}-l\right)+\delta\left(i_{-}+l\right)+\delta\left(i_{-}-l\right)\right]\left[\delta \left(\tilde{i}_{+}\right.\right. \\
& \left.+l)+\delta\left(\tilde{i}_{+}-l\right)+\delta\left(\tilde{i}_{-}+l\right)+\delta\left(\tilde{i}_{-}-l\right)\right] G_{0}(q+k, l) G_{0}(q, 0)+\frac{1}{4} \sum_{l_{1}, l_{2}=1}^{\infty}\left[\delta\left(i_{+}+l_{1}+l_{2}\right)\right. \\
& +\delta\left(i_{+}-l_{1}+l_{2}\right)+\delta\left(i_{+}+l_{1}-l_{2}\right)+\delta\left(i_{+}-l_{1}-l_{2}\right)+\delta\left(i_{-}+l_{1}+l_{2}\right)+\delta\left(i_{-}-l_{1}+l_{2}\right) \\
& \left.+\delta\left(i_{-}+l_{1}-l_{2}\right)+\delta\left(i_{-}-l_{1}-l_{2}\right)\right]\left[\delta\left(\tilde{i}_{+}+l_{1}+l_{2}\right)+\delta\left(\tilde{i}_{+}-l_{1}+l_{2}\right)+\delta\left(\tilde{i}_{+}+l_{1}-l_{2}\right)\right. \\
& \left.+\delta\left(\tilde{i}_{+}-l_{1}-l_{2}\right)+\delta\left(\tilde{i}_{-}+l_{1}+l_{2}\right)+\delta\left(\tilde{i}_{-}-l_{1}+l_{2}\right)+\delta\left(\tilde{i}_{-}+l_{1}-l_{2}\right)+\delta\left(\tilde{i}_{-}-l_{1}-l_{2}\right)\right] \times \\
& \left.G_{0}\left(q+k, l_{1}\right) G_{0}\left(q, l_{2}\right)\right) .
\end{aligned}
$$

The summation involving the combination $\left[\delta\left(i_{ \pm}+l\right)+\delta\left(i_{ \pm}-l\right)\right]$ can be transformed into one whose range extends to negative integer values not including the zero. Indeed, the identity

$$
\begin{aligned}
& \sum_{l=1}^{\infty}\left[\delta\left(i_{ \pm}+l\right)+\delta\left(i_{ \pm}-l\right)\right]\left[\delta\left(\tilde{i}_{+}+l\right)+\delta\left(\tilde{i}_{+}-l\right)+\delta\left(\tilde{i}_{-}+l\right)+\delta\left(\tilde{i}_{-}-l\right)\right]=\sum_{l \neq 0, l=-\infty}^{\infty} \delta\left(i_{ \pm}\right. \\
& +l)\left[\delta\left(\tilde{i}_{+}+l\right)+\delta\left(\tilde{i}_{+}-l\right)+\delta\left(\tilde{i}_{-}+l\right)+\delta\left(\tilde{i}_{-}-l\right)\right],
\end{aligned}
$$

can be used so that

$$
\begin{aligned}
& \int d^{d-1} q \sum_{l=1}^{\infty}\left[\delta\left(i_{ \pm}+l\right)+\delta\left(i_{ \pm}-l\right)\right]\left[\delta\left(\tilde{i}_{+}+l\right)+\delta\left(\tilde{i}_{+}-l\right)+\delta\left(\tilde{i}_{-}+l\right)+\delta\left(\tilde{i}_{-}-l\right)\right] G_{0}(q+k, l) \\
& G_{0}(q, 0)=\int d^{d-1} q \sum_{l=-\infty}^{\infty}\left[\delta\left(i_{+}+l\right)+\delta\left(i_{-}-l\right)\right]\left[\delta\left(\tilde{i}_{+}+l\right)+\delta\left(\tilde{i}_{+}-l\right)+\delta\left(\tilde{i}_{-}+l\right)+\delta\left(\tilde{i}_{-}-l\right)\right] \\
& G_{0}(q+k, l) G_{0}(q, 0)-2\left[\delta\left(i_{+}\right)+\delta\left(i_{-}\right)\right]\left[\delta\left(\tilde{i}_{+}\right)+\delta\left(\tilde{i}_{-}\right)\right] \int d^{d-1} q G_{0}(q+k, 0) G_{0}(q, 0)
\end{aligned}
$$


Using these expressions inside the double summation and carrying out the computations for it in a similar way, we obtain the result in terms of summations with indices varying in the range $(-\infty, \infty)$. The single summation is cancelled by the zero mode of each index in the double summation and we are left with the following expression for this diagram

$$
\begin{aligned}
& \mathcal{O}=\frac{(N+8)}{9} \tilde{\sigma}^{2} \sum_{l_{1}, l_{2}=-\infty}^{\infty}\left[\delta\left(i_{+}+l_{1}+l_{2}\right)+\delta\left(i_{-}+l_{1}+l_{2}\right)\right]\left[\delta\left(\tilde{i}_{+}+l_{1}+l_{2}\right)+\delta\left(\tilde{i}_{+}-l_{1}\right.\right. \\
& \left.+l_{2}\right)+\delta\left(\tilde{i}_{+}+l_{1}-l_{2}\right)+\delta\left(\tilde{i}_{+}-l_{1}-l_{2}\right)+\delta\left(\tilde{i}_{-}+l_{1}+l_{2}\right)+\delta\left(\tilde{i}_{-}-l_{1}+l_{2}\right)+\delta\left(\tilde{i}_{-}+l_{1}-l_{2}\right) \\
& \left.+\delta\left(\tilde{i}_{-}-l_{1}-l_{2}\right)\right] \int d^{d-1} q G_{0}\left(q+k, l_{1}\right) G_{0}\left(q, l_{2}\right) .
\end{aligned}
$$

This form can be further reduced in such a way that at maximum one summation for each loop graph takes place. We can perform the summations very easily and express the diagram in the simpler form

$$
\begin{aligned}
& \mathcal{X}=\frac{(N+8)}{9} \tilde{\sigma}\left\{\left[\delta\left(i_{+}+\tilde{i}_{+}\right)+\delta\left(i_{+}-\tilde{i}_{+}\right)+\delta\left(i_{+}+\tilde{i}_{-}\right)+\delta\left(i_{+}-\tilde{i}_{-}\right)\right] I_{2}\left(k, i_{+} ; \tilde{\sigma}, \mu_{0}\right)\right. \\
& +\left[\delta\left(i_{-}+\tilde{i}_{+}\right)+\delta\left(i_{-}-\tilde{i}_{+}\right)+\delta\left(i_{-}+\tilde{i}_{-}\right)+\delta\left(i_{-}-\tilde{i}_{-}\right)\right] I_{2}\left(k, i_{-} ; \tilde{\sigma}, \mu_{0}\right)+\tilde{I}_{2}\left(k, \frac{i_{+}+\tilde{i}_{+}}{2}, \frac{i_{+}-\tilde{i}_{+}}{2},\right. \\
& \left.\tilde{\sigma}, \mu_{0}\right)+\tilde{I}_{2}\left(k, \frac{i_{+}-\tilde{i}_{+}}{2}, \frac{i_{+}+\tilde{i}_{+}}{2}, \tilde{\sigma}, \mu_{0}\right)+\tilde{I}_{2}\left(k, \frac{i_{+}+\tilde{i}_{-}}{2}, \frac{i_{+}-\tilde{i}_{-}}{2}, \tilde{\sigma}, \mu_{0}\right)+\tilde{I}_{2}\left(k, \frac{i_{+}-\tilde{i}_{-}}{2},\right. \\
& \left.\frac{i_{+}+\tilde{i}_{-}}{2}, \tilde{\sigma}, \mu_{0}\right)+\tilde{I}_{2}\left(k, \frac{i_{-}+\tilde{i}_{+}}{2}, \frac{i_{-}-\tilde{i}_{+}}{2}, \tilde{\sigma}, \mu_{0}\right)+\tilde{I}_{2}\left(k, \frac{i_{-}-\tilde{i}_{+}}{2}, \frac{i_{-}+\tilde{i}_{+}}{2}, \tilde{\sigma}, \mu_{0}\right)+\tilde{I}_{2}(k \\
& \left.\left.\frac{i_{-}+\tilde{i}_{-}}{2}, \frac{i_{-}-\tilde{i}_{-}}{2}, \tilde{\sigma}, \mu_{0}\right)+\tilde{I}_{2}\left(k, \frac{i_{-}-\tilde{i}_{-}}{2}, \frac{i_{-}+\tilde{i}_{-}}{2}, \tilde{\sigma}, \mu_{0}\right)\right\}
\end{aligned}
$$

where

$$
\begin{aligned}
& I_{2}\left(k, i, \tilde{\sigma}, \mu_{0}\right)=\tilde{\sigma} \sum_{l=-\infty}^{\infty} \int \frac{d^{d-1} q}{\left[q^{2}+\tilde{\sigma}^{2} l^{2}+\mu_{0}^{2}\right]\left[(q+k)^{2}+\tilde{\sigma}^{2}(l+i)^{2}+\mu_{0}^{2}\right]}, \\
& \tilde{I}_{2}\left(k, i, j, \tilde{\sigma}, \mu_{0}\right)=\tilde{\sigma} \int \frac{d^{d-1} q}{\left[q^{2}+\tilde{\sigma}^{2} i^{2}+\mu_{0}^{2}\right]\left[(q+k)^{2}+\tilde{\sigma}^{2} j^{2}+\mu_{0}^{2}\right]} .
\end{aligned}
$$

For $D B C$, the absence of zero modes in the four-index tensor makes the computation easier. Now the diagram reads:

$$
\chi=\frac{(N+8)}{9} \lambda_{0}^{2} \sum_{l_{1}, l_{2}=1}^{\infty} \tilde{S}_{i_{1} i_{2} l_{1} l_{2}}^{(-1)} \tilde{S}_{l_{1} l_{2} i_{3} i_{4}}^{(-1)} \int d^{d-1} q G_{0}\left(q+k, l_{1}\right) G_{0}\left(q, l_{2}\right),
$$

and we have to transform the summation to the range $(-\infty, \infty)$ just as before after using the tensor components for $\tau=-1$. The unified form holding for both $\tau=-1(D B C)$ and 
$\tau=1(N B C)$ can be written as

$$
\begin{aligned}
& \Upsilon \mathcal{X}=\frac{(N+8)}{9} \tilde{\sigma}\left\{\left[\delta\left(i_{+}+\tilde{i}_{+}\right)+\delta\left(i_{+}-\tilde{i}_{+}\right)+\tau\left(\delta\left(i_{+}+\tilde{i}_{-}\right)+\delta\left(i_{+}-\tilde{i}_{-}\right)\right)\right] I_{2}\left(k, i_{+} ; \tilde{\sigma}, \mu_{0}\right)\right. \\
& +\left[\tau\left(\delta\left(i_{-}+\tilde{i}_{+}\right)+\delta\left(i_{-}-\tilde{i}_{+}\right)\right)+\delta\left(i_{-}+\tilde{i}_{-}\right)+\delta\left(i_{-}-\tilde{i}_{-}\right)\right] I_{2}\left(k, i_{-} ; \tilde{\sigma}, \mu_{0}\right)+\tau\left[\tilde { I } _ { 2 } \left(k, \frac{i_{+}-\tilde{i}_{+}}{2},\right.\right. \\
& \left.\frac{i_{+}+\tilde{i}_{+}}{2}, \tilde{\sigma}, \mu_{0}\right)+\tilde{I}_{2}\left(k, \frac{i_{+}+\tilde{i}_{+}}{2}, \frac{i_{+}-\tilde{i}_{+}}{2}, \tilde{\sigma}, \mu_{0}\right)+\tilde{I}_{2}\left(k, \frac{i_{-}+\tilde{i}_{-}}{2}, \frac{i_{-}-\tilde{i}_{-}}{2}, \tilde{\sigma}, \mu_{0}\right)+\tilde{I}_{2}(k, \\
& \left.\left.\frac{i_{-}-\tilde{i}_{-}}{2}, \frac{i_{-}+\tilde{i}_{-}}{2}, \tilde{\sigma}, \mu_{0}\right)\right]+\tilde{I}_{2}\left(k, \frac{i_{-}+\tilde{i}_{+}}{2}, \frac{i_{-}-\tilde{i}_{+}}{2}, \tilde{\sigma}, \mu_{0}\right)+\tilde{I}_{2}\left(k, \frac{i_{-}-\tilde{i}_{+}}{2}, \frac{i_{-}+\tilde{i}_{+}}{2}, \tilde{\sigma}, \mu_{0}\right) \\
& \left.+\tilde{I}_{2}\left(k, \frac{i_{+}-\tilde{i}_{-}}{2}, \frac{i_{+}+\tilde{i}_{-}}{2}, \tilde{\sigma}, \mu_{0}\right)+\tilde{I}_{2}\left(k, \frac{i_{+}+\tilde{i}_{-}}{2}, \frac{i_{+}-\tilde{i}_{-}}{2}, \tilde{\sigma}, \mu_{0}\right)\right\},
\end{aligned}
$$

with the integrals defined by Eqs. (26). From this simple analysis, we conclude that the Feynman diagrams in the exponential representation involve more complicated objects like the "nondiagonal" integrals $\tilde{I}_{2}\left(k, i, j, \tilde{\sigma}, \mu_{0}\right)$. Consequently, each momentum loop integral in the finite system for $D B C$ and $N B C$ cannot be obtained from the infinite system through the substitution $\int d^{d} k \rightarrow \sum_{j=-\infty}^{\infty} \sigma \int d^{d-1} k$ as in $P B C$ and $A B C$. The higher the loop graph,

the lengthier is the computation of the contractions of $\tilde{S}_{j_{1} j_{2} j_{3} j_{4}}^{(\tau)}$ giving the particular diagram. Nevertheless, the same structure is preserved as in the simple one-loop examples just worked out. We postpone the presentation of all relevant higher loop diagrams after our next task, namely, the discussion of vertex parts that can be renormalized multiplicatively and include composite operators.

\section{The vertex $\Gamma^{(2,1)}$ : tree-level and one-loop graph}

The most nontrivial feature of the construction proposed in the present work manifests itself in the diagrams of the composed field. The reason for that are the basis functions of the composite fields associated to the $\Gamma^{(2,1)}$ vertex part (and its descendents that can be renormalized multiplicatively), which consists of one single type for Dirichlet and Neumann boundary conditions.

Applying the operator $\left(\nabla^{2}-\mu_{0}^{2}\right)$ to the composite field $\left(\phi_{0}^{(\tau)}(x)\right)^{2}$ and using the field equations $\left(\nabla^{2}-\mu_{0}^{2}\right) \phi_{0}^{(\tau)}(x)=0$, we find

$$
\left(\nabla^{2}-\mu_{0}^{2}\right)\left(\phi_{0}^{(\tau)}(x)\right)^{2}=2\left(\nabla \phi_{0}^{(\tau)}(x)\right)^{2}
$$


The composite field also satisfies $D B C$ and $N B C$, respectively, namely

$$
\begin{gathered}
\left.\left(\phi_{0}^{(\tau=-1)}(x)\right)^{2}\right|_{z=0, L}=0, \\
\left.\frac{\partial}{\partial z}\left(\phi_{0}^{(\tau=1)}(x)\right)^{2}\right|_{z=0, L}=0 .
\end{gathered}
$$

We decompose the composite field in terms of its components in momentum space as $\left(\phi_{0}^{(\tau)}(x)\right)^{2}=\sum_{j} \int d^{d-1} \operatorname{pexp}(i \vec{p} . \vec{\rho}) \tilde{u}_{j}^{(\tau)}(z)\left(\phi_{0 j}^{(\tau)}(\vec{p}, j)\right)^{2}$. Our goal is the determination of the new basis functions of the composite field $\tilde{u}_{j}^{(\tau)}(z)$ preferably in terms of the previous basis functions expressed in terms of sines and cosines. In order to achieve that, we first work out the representation of the composite field in connection with the product of two single fields computed at the same point, apply the differential operators over them and examine the consequences. Afterwards, we compare the results of the same operation performed on the above definition of the composite fields and the basis functions $\tilde{u}_{j}^{(\tau)}(z)$.

Using the representation of the field and taking the product of two fields at the same point, we find $\phi_{0}(x) \phi_{0}(x)=\sum_{j_{1}, j_{2}} \int d^{d-1} k_{1} \exp \left(i\left(\vec{k}_{1}+\vec{k}_{2}\right) \cdot \vec{\rho}\right) u_{j_{1}}(z) u_{j_{2}}(z) \phi_{0 j_{1}}\left(\vec{k}_{1}\right) \phi_{0 j_{2}}\left(\vec{k}_{2}\right)$. Recalling that $\nabla^{2}=\frac{\partial^{2}}{\partial \rho^{2}}+\frac{\partial^{2}}{\partial z^{2}}$, applying this operator to the product of two fields and inserting this in above equation, we find

$$
\left[\frac{\partial^{2}}{\partial z^{2}}-\left(p_{1}^{2}+p_{2}^{2}\right)-2 \mu_{0}^{2}\right] u_{j_{1}}^{(\tau)}(z) u_{j_{2}}^{(\tau)}(z)=2 \frac{d u_{j_{1}}^{(\tau)}}{d z} \frac{d u_{j_{2}}^{(\tau)}}{d z}
$$

Restricting ourselves only to nonvanishing values for $j_{1}, j_{2}$, we can show that $u_{j_{1}}^{(\tau)}(z) u_{j_{2}}^{(\tau)}(z)=$ $\frac{1}{L}\left[\cos \left(\tilde{\sigma}\left(j_{1}-j_{2}\right) z\right)+\tau \cos \left(\tilde{\sigma}\left(j_{1}+j_{2}\right) z\right)\right]$. This implies that $\frac{d u_{j_{1}}^{(\tau)}}{d z} \frac{d u_{j_{2}}^{(\tau)}}{d z}=\frac{\tilde{\sigma}^{2} j_{1} j_{2}}{L}\left[\cos \left(\tilde{\sigma}\left(j_{1}-j_{2}\right) z\right)-\right.$ $\left.\tau \cos \left(\tilde{\sigma}\left(j_{1}+j_{2}\right) z\right)\right]$. Using the definition $\tilde{u}_{j_{1}, j_{2}} \equiv u_{j_{1}}^{(\tau)}(z) u_{j_{2}}^{(\tau)}(z)$ into the above equation leads us to the identity $\left(p_{1}^{2}+p_{2}^{2}+\tilde{\sigma}^{2} j_{1}^{2}+\tilde{\sigma}^{2} j_{1}^{2}+2 \mu_{0}^{2}\right) \tilde{u}_{j_{1}, j_{2}}=0$. These manipulations suggest the choice for the basis functions of the composite field:

$$
\tilde{u}_{j}^{\tau}(z)=\frac{A}{L} \cos (\tilde{\sigma} j z)
$$

which implies that $\tilde{u}_{j_{1}, j_{2}}=\frac{1}{A}\left[\tilde{u}_{j_{1}-j_{2}}+\tau \tilde{u}_{j_{1}+j_{2}}\right]$. We can now determine the tensor associated to the vertex part $\Gamma^{(2,1)}$. The typical object responsible for the appearance of these diagrams corresponds to

$$
F^{(2,1)}=\int d^{d-1} \rho \int_{0}^{L} d z \phi_{0}^{(\tau)}(x) \phi_{0}^{(\tau)}(x)\left(\phi_{0}^{(\tau)}(x)\right)^{2} .
$$

Replacing the Fourier expansion of the fields and composite operator, we obtain

$$
F^{(2,1)}=\sum_{j_{1}, j_{2}, j} \hat{S}_{j_{1} j_{2} j}^{(\tau)} \int d^{d-1} p_{1} d^{d-1} p_{2} d^{d-1} p \delta^{d-1}\left(\vec{p}_{1}+\vec{p}_{2}+\vec{p}\right) \phi_{0 j_{1}}\left(\vec{p}_{1}\right) \phi_{0 j_{2}}\left(\vec{p}_{2}\right)\left(\phi_{0 j}(\vec{p})\right)^{2}
$$


where

$$
\hat{S}_{j_{1} j_{2} j}^{(\tau)}=\int_{0}^{L} u_{j_{1}}^{(\tau)}(z) u_{j_{2}}^{(\tau)}(z) \tilde{u}_{j}^{(\tau)}(z) d z .
$$

The constant $A$ can be determined by imposing the normalization condition $\int_{0}^{L} \tilde{u}_{j}(z) \tilde{u}_{j^{\prime}}(z) d z=\frac{L}{2}\left[\delta\left(j-j^{\prime}\right)+\delta\left(j+j^{\prime}\right)\right]$ with $j, j^{\prime} \neq 0$, which implies $A=L$. Therefore, it can be checked that

$$
\hat{S}_{j_{1} j_{2} j}^{(\tau)}=\frac{1}{2}\left[\delta\left(j-j_{1}+j_{2}\right)+\delta\left(j+j_{1}-j_{2}\right)+\tau \delta\left(j+j_{1}+j_{2}\right)+\tau \delta\left(j-j_{1}-j_{2}\right)\right] .
$$

We can invert the Fourier transform from the product of two fields computed at the same point, using the identity $\tilde{u}_{j_{1}, j_{2}}=\frac{1}{L}\left[\tilde{u}_{j_{1}-j_{2}}+\tau \tilde{u}_{j_{1}+j_{2}}\right]$. First, we multiply it by $e^{-i \vec{p} . \vec{\rho}}$ and integrate over $d^{d-1} \rho$. After that, multiply the resulting expression by $\tilde{u}_{j^{\prime}}(z)$ and integrate over $z$ using the orthonormality conditions of $\tilde{u}_{j}(z)$. We obtain:

$$
\phi_{0}^{(\tau)}(\vec{p}, j)=\frac{1}{L} \int d^{d-1} \rho\left[\phi_{0}^{(\tau)}(x)\right]^{2} \exp [-i \vec{p} . \vec{\rho}] \int_{0}^{L} \tilde{u}_{j}(z) d z .
$$

Replacing this result in the left-hand side of the expression which defines the Fourier transform of the composite field (with only one summation), we get to the closure relation $\frac{1}{L} \sum_{j} \tilde{u}_{j}(z) \tilde{u}_{j}\left(z^{\prime}\right)=\delta\left(z-z^{\prime}\right)$. This completes the basic properties of the basis functions of the composite field.

Before analyzing the situation for $N B C$ let us make an important remark concerning the construction of arbitrary loop diagrams for the composite field. First, observe that even though this tensor is similar to the four-point vertex part tensor $\tilde{S}_{j_{1} j_{2} j_{3} j_{4}}^{(\tau)}$, the similarity is not complete. We would like to make a connection between the tensors $\hat{S}_{j_{1} j_{2} j}^{(\tau)}$ and $\tilde{S}_{j_{1} j_{2} j_{3} j_{4}}^{(\tau)}$, or in other words, the graphs of $\Gamma^{(2,1)}$ with those from $\Gamma^{(4)}$ by identifying, for instance two external legs of the latter with the composite field insertion, as is usual for infinite systems.

The fact of the matter is that a simple way to link the two types of graphs which generalizes the situation of bulk systems is to perform identifications between the external quasi-momenta. If we set $j_{3}=j_{4}=j^{\prime}$, we find $\tilde{S}_{j_{1} j_{2} j^{\prime} j^{\prime}}^{(\tau)}=\tilde{\sigma}\left[\delta\left(2 j^{\prime}+j_{1}+j_{2}\right)+2 \tau \delta\left(j_{1}+j_{2}\right)+\right.$ $\left.\delta\left(2 j^{\prime}-j_{1}-j_{2}\right)+\tau \delta\left(2 j^{\prime}-j_{1}+j_{2}\right)+\tau \delta\left(2 j^{\prime}+j_{1}-j_{2}\right)+2 \delta\left(j_{1}-j_{2}\right)\right]$ (no summation in the repeated index).

Looking at Eq. (36), it is tempting to perform the identification $2 j^{\prime} \equiv j$ in that formula, but this does not solve the problem completely, for the additional contributions showing up in $\tilde{S}_{j_{1} j_{2} j^{\prime} j^{\prime}}^{(\tau)}$ (proportional to $\left.\delta\left(j_{1} \pm j_{2}\right)\right)$ are absent in $\hat{S}_{j_{1} j_{2} j}^{(\tau)}$. Since the moding of the composite operator index can take zero values for both $N B C$ and $D B C$, the construction of the $\Gamma^{(2,1)}$ 
graphs with the tensor $\hat{S}_{j_{1} j_{2} j}^{(\tau)}$ needs to be modified. In fact, the right combination to form an arbitrary loop graph should include the $j=0$ and $j \neq 0$ pieces components from $\hat{S}_{j_{1} j_{2} j}^{(\tau)}$. To be precise, an arbitrary graph always contains the contributions proportional to $\hat{S}_{j_{1} j_{2} j}^{(\tau)}+\tau \hat{S}_{j_{1} j_{2} 0}^{(\tau)}$ $\left(j= \pm\left(j_{1}+j_{2}\right)\right)$ contracted with $\tilde{S}_{j_{1} j_{2} j_{3} j_{4}}^{(\tau)}$ tensor stemming from the perturbative expansion in the coupling constant. In terms of the diagram the combination that should appear is

$$
\sum_{1--\varepsilon_{-2}^{3}}^{3}=\left.\sum_{1--\varepsilon_{-2}}^{3}\right|_{\left(j= \pm\left(j_{1}+j_{2}\right)\right)}+\left.\tau \sum_{1--\sum_{-2}}^{3}\right|_{(j=0)},
$$

where the sign of $j$ is fixed. With this recipe utilized in the construction of each loop diagram, the renormalization of the composite field can be performed in a simple manner, as we are going to discuss later on.

Let us focus now on the zero modes in Neumann boundary conditions whenever $j_{1}=$ $0\left(j_{2} \neq 0\right), j_{2}=0\left(j_{1} \neq 0\right)$ and $j_{1}=j_{2}=0$ present in the tensor $\hat{S}_{j_{1} j_{2} j}^{(\tau)}$. First, note that the zero mode composite basis functions are the same, namely, $\tilde{u}^{(1)}=\cos (\tilde{\sigma} j z)$ and the product involving a zero mode becomes $\tilde{u}_{0, j}^{(1)}(z)=u_{0}^{(1)} u_{j}^{(1)}=\frac{\sqrt{2}}{L} \cos (\tilde{\sigma} j z)$. From the definition $\hat{S}_{0 j_{2 j}}^{(1)}=$ $\int_{0}^{L} u_{0}^{(1)}(z) u_{j_{2}}^{(1)}(z) \tilde{u}_{j}^{(1)}(z) d z$ we read off the values $\hat{S}_{0 j_{2} j}^{(1)}=\hat{S}_{j_{2} 0 j}^{(1)}=\frac{1}{\sqrt{2}}\left[\delta\left(j+j_{2}\right)+\delta\left(j-j_{2}\right)\right]$. Analogously, it is not difficult to prove that $\hat{S}_{00 j}^{(1)}=\delta(j)$.

We can employ the framework just developed to compute arbitrary loop diagrams including multiplicatively renormalizable composite vertex operators. At the present moment we shall restrict ourselves in getting the expression corresponding to the one-loop graph for $\Gamma^{(2,1)}$. It is represented by

$$
\bigodot^{\sum_{3}}=\frac{(N+2)}{6} \sum_{j_{1}, j_{2} \geq 0}^{\infty} \tilde{S}_{i_{1} i_{2} j_{1} j_{2}}^{(\tau)} \hat{S}_{j_{1} j_{2} j}^{(\tau)} \int d^{d-1} q G_{0}\left(q+k, j_{1}\right) G_{0}\left(q, j_{2}\right),
$$

Keep in mind that we wish to express this diagram into a unified form. Therefore, we choose $i_{1}, i_{2} \neq 0$. Let us start explicitly with Neumann boundary conditions. The summations split in the form:

$$
\begin{aligned}
& \sum_{j_{1}, j_{2} \geq 0}^{\infty} \tilde{S}_{i_{1} i_{2} j_{1} j_{2}}^{(1)} \hat{S}_{j_{1} j_{2} j}^{(1)} \int d^{d-1} q G_{0}\left(q+k, j_{1}\right) G_{0}\left(q, j_{2}\right)=\int d^{d-1} q\left[\tilde{S}_{i_{1} i_{2} 00}^{(1)} \hat{S}_{00 j}^{(1)} G_{0}(q+k, 0) G_{0}(q, 0)\right. \\
& +\sum_{j_{1}=1}^{\infty} \tilde{S}_{i_{1} i_{2} j_{1} 0}^{(1)} \hat{S}_{j_{1} 0 j}^{(1)} G_{0}\left(q+k, j_{1}\right) G_{0}(q, 0)+\sum_{j_{2}=1}^{\infty} \tilde{S}_{i_{1} i_{2} 0 j_{2}}^{(1)} \hat{S}_{0 j_{2} j}^{(1)} G_{0}(q+k, 0) G_{0}\left(q, j_{2}\right)+\sum_{j_{1}, j_{2}=1}^{\infty} \tilde{S}_{i_{1} i_{2} j_{1} j_{2}}^{(1)} \\
& \left.\times \hat{S}_{j_{1} j_{2} j}^{(1)} G_{0}\left(q+k, j_{1}\right) G_{0}\left(q, j_{2}\right)\right] .
\end{aligned}
$$


Using explicitly the tensor components we first obtain $\left(i_{ \pm} \equiv i_{1} \pm i_{2}\right)$ :

$$
\begin{aligned}
& \sum_{j_{1}, j_{2} \geq 0}^{\infty} \tilde{S}_{i_{1} i_{2} j_{1} j_{2}}^{(1)} \hat{S}_{j_{1} j_{2} j}^{(1)} \int d^{d-1} q G_{0}\left(q+k, j_{1}\right) G_{0}\left(q, j_{2}\right)=\frac{\tilde{\sigma}}{2} \int d^{d-1} q\left[2 \sum_{j_{1}=1}^{\infty}\left[\delta\left(j-j_{1}\right)+\delta\left(j+j_{1}\right)\right]\right. \\
& {\left[\delta\left(i_{+}+j_{1}\right)+\delta\left(i_{+}-j_{1}\right)+\delta\left(i_{-}+j_{1}\right)+\delta\left(i_{-}-j_{1}\right)\right] G_{0}\left(q+k, j_{1}\right) G_{0}(q, 0)+2 \sum_{j_{2}=1}^{\infty}\left[\delta\left(j-j_{2}\right)\right.} \\
& \left.+\delta\left(j+j_{2}\right)\right]\left[\delta\left(i_{+}+j_{2}\right)+\delta\left(i_{+}-j_{2}\right)+\delta\left(i_{-}+j_{2}\right)+\delta\left(i_{-}-j_{2}\right)\right] G_{0}(q+k, 0) G_{0}\left(q, j_{2}\right) \\
& +\sum_{j_{1}, j_{2}=1}^{\infty}\left[\delta\left(i_{+}+j_{1}+j_{2}\right)+\delta\left(i_{+}-j_{1}+j_{2}\right)+\delta\left(i_{+}+j_{1}-j_{2}\right)+\delta\left(i_{+}-j_{1}-j_{2}\right)+\delta\left(i_{-}+j_{1}\right.\right. \\
& \left.\left.+j_{2}\right)+\delta\left(i_{-}-j_{1}+j_{2}\right)+\delta\left(i_{-}+j_{1}-j_{2}\right)+\delta\left(i_{-}-j_{1}-j_{2}\right)\right]\left[\delta\left(j+j_{1}+j_{2}\right)+\delta\left(j-j_{1}+j_{2}\right)\right. \\
& \left.+\delta\left(j+j_{1}-j_{2}\right)+\delta\left(j-j_{1}-j_{2}\right)\right] G_{0}\left(q+k, j_{1}\right) G_{0}\left(q, j_{2}\right)+4 \delta(j)\left[\delta\left(i_{+}\right)+\delta\left(i_{-}\right)\right] G_{0}(q, 0) \\
& \left.\left.\times G_{0}(q+k, 0)\right]\right] .
\end{aligned}
$$

In the first term of the first (second) single summation we perform the change $j_{1} \rightarrow-j_{1}\left(j_{2} \rightarrow\right.$ $\left.-j_{2}\right)$. This extends the range of the summation to the interval $(-\infty, \infty)$ but precluding the zero mode. We can include it in order to write the first summation as:

$$
\begin{aligned}
& \sum_{j_{1}=1}^{\infty}\left[\delta\left(j-j_{1}\right)+\delta\left(j+j_{1}\right)\right]\left[\delta\left(i_{+}+j_{1}\right)+\delta\left(i_{+}-j_{1}\right)+\delta\left(i_{-}+j_{1}\right)+\delta\left(i_{-}-j_{1}\right)\right] G_{0}\left(q+k, j_{1}\right) \\
& G_{0}(q, 0)=\sum_{j_{1}=-\infty}^{\infty} \delta\left(j+j_{1}\right)\left[\delta\left(i_{+}+j_{1}\right)+\delta\left(i_{+}-j_{1}\right)+\delta\left(i_{-}+j_{1}\right)+\delta\left(i_{-}-j_{1}\right)\right] G_{0}\left(q+k, j_{1}\right) \\
& \quad \times G_{0}(q, 0)-2 \delta(j)\left[\delta\left(i_{+}\right)+\delta\left(i_{-}\right)\right] G_{0}(q+k, 0) G_{0}(q, 0),
\end{aligned}
$$

and similarly for the second single summation. The double summation can be manipulated using analogous steps and leads to

$$
\begin{aligned}
& \sum_{j_{1}, j_{2}=1}^{\infty}\left[\delta\left(i_{+}+j_{1}+j_{2}\right)+\delta\left(i_{+}-j_{1}+j_{2}\right)+\delta\left(i_{+}+j_{1}-j_{2}\right)+\delta\left(i_{+}-j_{1}-j_{2}\right)+\delta\left(i_{-}+j_{1}+j_{2}\right)\right. \\
& \left.+\delta\left(i_{-}-j_{1}+j_{2}\right)+\delta\left(i_{-}+j_{1}-j_{2}\right)+\delta\left(i_{-}-j_{1}-j_{2}\right)\right]\left[\delta\left(j+j_{1}+j_{2}\right)+\delta\left(j-j_{1}+j_{2}\right)+\delta(j\right. \\
& \left.\left.+j_{1}-j_{2}\right)+\delta\left(j-j_{1}-j_{2}\right)\right] G_{0}\left(q+k, j_{1}\right) G_{0}\left(q, j_{2}\right)=\sum_{j_{1}, j_{2}=-\infty}^{\infty}\left[\delta\left(i_{+}+j_{1}+j_{2}\right)+\delta\left(i_{+}-j_{1}+j_{2}\right)\right. \\
& +\delta\left(i_{+}+j_{1}-j_{2}\right)+\delta\left(i_{+}-j_{1}-j_{2}\right)+\delta\left(i_{-}+j_{1}+j_{2}\right)+\delta\left(i_{-}-j_{1}+j_{2}\right)+\delta\left(i_{-}+j_{1}-j_{2}\right) \\
& \left.+\delta\left(i_{-}-j_{1}-j_{2}\right)\right] \delta\left(j+j_{1}+j_{2}\right) G_{0}\left(q+k, j_{1}\right) G_{0}\left(q, j_{2}\right)-2 \sum_{j_{1}=-\infty}^{\infty} \delta\left(j+j_{1}\right)\left[\delta\left(i_{+}+j_{1}\right)+\delta\left(i_{+}\right.\right. \\
& \left.\left.-j_{1}\right)+\delta\left(i_{-}+j_{1}\right)+\delta\left(i_{-}-j_{1}\right)\right] G_{0}\left(q+k, j_{1}\right) G_{0}(q, 0)-2 \sum_{j_{2}=-\infty}^{\infty} \delta\left(j+j_{2}\right)\left[\delta\left(i_{+}+j_{2}\right)+\delta\left(i_{+}\right.\right. \\
& \left.\left.-j_{2}\right)+\delta\left(i_{-}+j_{2}\right)+\delta\left(i_{-}-j_{2}\right)\right] G_{0}(q+k, 0) G_{0}\left(q, j_{2}\right)+4 \delta(j)\left[\delta\left(i_{+}\right)+\delta\left(i_{-}\right)\right] G_{0}(q+k, 0) \\
& \times G_{0}(q, 0) .
\end{aligned}
$$


Replacing the value of each expression inside the combination of the diagram, the single summations and the independent terms cancel among each other and we obtain:

$$
\begin{aligned}
& \bigodot_{\left.-j_{2}\right)+\delta}^{\sum_{0}}=\frac{(N+2)}{6} \frac{\tilde{\sigma}}{2} \sum_{j_{1}, j_{2}=-\infty}^{\infty} \delta\left(j+j_{1}+j_{2}\right)\left[\delta\left(i_{+}+j_{1}+j_{2}\right)+\delta\left(i_{+}-j_{1}+j_{2}\right)+\delta\left(i_{+}+j_{1}\right.\right. \\
& \left.\left.-j_{2}\right)\right] \int d^{d-1} q G_{0}\left(q+k, j_{1}\right) g_{1}\left(q, j_{2}\right) .
\end{aligned}
$$

Recall that we wish to express the one-loop diagram involving at maximum a single summation. Performing the summations explictly, we find

$$
\begin{aligned}
& \bigodot^{3}=\frac{(N+2)}{6} \frac{1}{2}\left[\left(\delta\left(i_{+}+j\right)+\delta\left(i_{+}-j\right)+\delta\left(i_{-}+j\right)+\delta\left(i_{-}-j\right)\right) I_{2}\left(k, j, \tilde{\sigma}, \mu_{0}\right)\right. \\
& +\tilde{I}_{2}\left(k, \frac{i_{+}-j}{2}, \frac{i_{+}+j}{2}, \tilde{\sigma}, \mu_{0}\right)+\tilde{I}_{2}\left(k, \frac{i_{+}+j}{2}, \frac{i_{+}-j}{2}, \tilde{\sigma}, \mu_{0}\right)+\tilde{I}_{2}\left(k, \frac{i_{-}-j}{2}, \frac{i_{-}+j}{2}, \tilde{\sigma}, \mu_{0}\right) \\
& \left.+\tilde{I}_{2}\left(k, \frac{i_{-}+j}{2}, \frac{i_{-}-j}{2}, \tilde{\sigma}, \mu_{0}\right)\right],
\end{aligned}
$$

where the integrals $I_{2}\left(\left(k, i, \tilde{\sigma}, \mu_{0}\right)\right.$ and $\tilde{I}_{2}\left(k, i, j, \tilde{\sigma}, \mu_{0}\right)$ are defined in Eqs. (26).

For $D B C$ the situation is even simpler since we do not have to deal with zero modes. We can write

$$
\bigodot^{3}=\frac{(N+2)}{6} \sum_{j_{1}, j_{2}=1}^{\infty} \tilde{S}_{i_{1} i_{2} j_{1} j_{2}}^{(-1)} \hat{S}_{j_{1} j_{2} j}^{(-1)} \int d^{d-1} q G_{0}\left(q+k, j_{1}\right) G_{0}\left(q, j_{2}\right) .
$$

Using the tensor components already derived, we can write the summation as

$$
\begin{aligned}
& \sum_{j_{1}, j_{2}=1}^{\infty} \tilde{S}_{i_{1} i_{2} j_{1} j_{2}}^{(1)} \hat{S}_{j_{1} j_{2} j}^{(1)} \int d^{d-1} q G_{0}\left(q+k, j_{1}\right) G_{0}\left(q, j_{2}\right)=\sum_{j_{1}, j_{2}=1}^{\infty}\left[\delta\left(i_{+}+j_{1}+j_{2}\right)+\delta\left(i_{+}-j_{1}\right.\right. \\
& \left.-j_{2}\right)-\delta\left(i_{-}+j_{1}+j_{2}\right)-\delta\left(i_{-}-j_{1}-j_{2}\right)-\delta\left(i_{+}-j_{1}+j_{2}\right)-\delta\left(i_{+}+j_{1}-j_{2}\right)+\delta\left(i_{-}-j_{1}\right. \\
& \left.\left.+j_{2}\right)+\delta\left(i_{-}+j_{1}-j_{2}\right)\right]\left[\delta\left(j-j_{1}+j_{2}\right)+\delta\left(j+j_{1}-j_{2}\right)-\delta\left(j-j_{1}-j_{2}\right)-\delta\left(j+j_{1}+j_{2}\right)\right] \\
& \times \int d^{d-1} q G_{0}\left(q+k, j_{1}\right) G_{0}\left(q, j_{2}\right) .
\end{aligned}
$$

Now, in the second bracket perform the change $j_{2} \rightarrow-j_{2}$ in the first and fourth terms. This has the effect to produce a global factor of $(-1)$ in each coefficient of those two terms. The net result can be written in the form

$$
\begin{aligned}
& \sum_{j_{1}, j_{2}=1}^{\infty} \tilde{S}_{i_{1} i_{2} j_{1} j_{2}}^{(1)} \hat{S}_{j_{1} j_{2} j}^{(1)} \int d^{d-1} q G_{0}\left(q+k, j_{1}\right) G_{0}\left(q, j_{2}\right)=-\sum_{j_{1}=1 j_{2}=-\infty, j_{2} \neq 0}^{\infty} \sum_{+j_{2}}^{\infty} \delta\left(j+j_{1}+j_{2}\right)\left[\delta \left(i_{+}+j_{1}\right.\right. \\
& \left.+j_{2}\right)+\delta\left(i_{+}-j_{1}-j_{2}\right)-\delta\left(i_{-}+j_{1}+j_{2}\right)-\delta\left(i_{-}-j_{1}-j_{2}\right)-\delta\left(i_{+}-j_{1}+j_{2}\right)-\delta\left(i_{+}+j_{1}-j_{2}\right) \\
& \left.+\delta\left(i_{-}-j_{1}+j_{2}\right)+\delta\left(i_{-}+j_{1}-j_{2}\right)\right] \int d^{d-1} q G_{0}\left(q+k, j_{1}\right) G_{0}\left(q, j_{2}\right) .
\end{aligned}
$$


By performing similar transformations in some terms involving $j_{1}\left(\rightarrow-j_{1}\right)$, we can extend the summation over $j_{1}$ for negative values as well. The simplicity here is that no zero mode survives when we include the values $j_{1}, j_{2}=0$ in the summations. The graph then reads:

$$
\begin{aligned}
& \int_{\left.-j_{2}\right)+\delta}=-\frac{(N+2)}{6} \frac{\tilde{\sigma}}{2} \sum_{j_{1}, j_{2}=-\infty}^{\infty} \delta\left(j+i_{+}-j_{1}+j_{2}\right)\left[\delta\left(i_{+}+j_{1}+j_{2}\right)-\delta\left(i_{+}-j_{1}+j_{2}\right)-\delta\left(i_{+}+j_{1}\right.\right. \\
& \left.\left.-j_{2}\right)\right] \int d^{d-1} q G_{0}\left(q+k, j_{2}\right)+\delta\left(i_{-}\right) G_{0}\left(q, j_{2}\right) .
\end{aligned}
$$

Comparing this with Eq. (44), $D B C(\tau=-1)$ and $N B C(\tau=1)$ can be unified very easily. When this diagram is written in terms of the integrals $I_{2}\left(k, i, \tilde{\sigma}, \mu_{0}\right)$ and $\tilde{I}_{2}\left(k, i, \tilde{\sigma}, \mu_{0}\right)$, the unified result is

$$
\begin{aligned}
& \bigodot_{0}^{\sum_{0}}=\frac{(N+2)}{6} \frac{\tau}{2}\left[\left(\delta\left(i_{+}+j\right)+\delta\left(i_{+}-j\right)+\tau\left(\delta\left(i_{-}+j\right)+\delta\left(i_{-}-j\right)\right)\right) I_{2}\left(k, j, \tilde{\sigma}, \mu_{0}\right)\right. \\
& +\tau\left[\tilde{I}_{2}\left(k, \frac{i_{+}-j}{2}, \frac{i_{+}+j}{2}, \tilde{\sigma}, \mu_{0}\right)+\tilde{I}_{2}\left(k, \frac{i_{+}+j}{2}, \frac{i_{+}-j}{2}, \tilde{\sigma}, \mu_{0}\right)\right]+\tilde{I}_{2}\left(k, \frac{i_{-}-j}{2}, \frac{i_{-}+j}{2}, \tilde{\sigma}, \mu_{0}\right) \\
& \left.+\tilde{I}_{2}\left(k, \frac{i_{-}+j}{2}, \frac{i_{-}-j}{2}, \tilde{\sigma}, \mu_{0}\right)\right] \text {. }
\end{aligned}
$$

This can be rewritten in a more elegant, compact notation as:

$$
\underset{\left.\left.\tilde{\sigma}, \mu_{0}\right)\right] .}{\sum_{0}} \frac{(N+2)}{6} \frac{\tau}{2} \sum_{\alpha, \beta=-1}^{1} \alpha^{\frac{1-\tau}{2}}\left[\delta\left(i_{\alpha}-\beta j\right) I_{2}\left(k, i_{\alpha}, \tilde{\sigma}, \mu_{0}\right)+\tau \tilde{I}_{2}\left(k, \frac{i_{\alpha}+\beta j}{2}, \frac{i_{\alpha}-\beta j}{2},\right.\right.
$$

This framework has the virtue of expressing all integrals in terms of infinite sums which makes it simple the comparison with the results from periodic and antiperiodic boundary conditions. Needless to say, all the massless integrals follow from the substitution $\mu_{0}=0$ in the integrals above and in the higher-loop contributions, which will be analyzed next.

3. Higher loop diagrams for $\Gamma^{(2)}, \Gamma^{(4)}$ and $\Gamma^{(2,1)}$

Our goal now is just to write down the various graphs in terms of integrals which resemble those from periodic and antiperiodic boundary conditions, possibly with additional "nondiagonal" terms. We are going to consider some two-loop graphs, although just a smaller subset of them will be necessary to our computation of the critical exponents within the 
present technique. We will analyze only one three-loop graph for the two-point function which is the only one needed for our purposes. We will save the construction of other nontrivial diagrams (external momentum dependent) including mass insertions for later when we will discuss the renormalization of this theory.

Start with the two point function $\Gamma^{(2)}$, for instance. We can classify the diagrams in trivial and nontrivial contributions. The trivial contributions will be generically called "tadpole diagrams" and are going to be discussed first. These contributions are displayed in the two-loop case for the sake of comparison with the one-loop case but we will not need them in the present computation.

Of course, we can start from scratch with two four-index finite-size tensors and perform the apropriate contractions between them in pretty much the same way we did in the oneloop case. The result including both boundary conditions is:

$$
\begin{aligned}
& \left\{=\left(\frac{N+2}{3}\right)^{2}\left[\delta\left(i_{-}\right) D_{2}\left(\tilde{\sigma}, \mu_{0}\right) D_{1}\left(\tilde{\sigma}, \mu_{0}\right)+\tau \tilde{I}_{2}\left(0, \frac{i_{-}}{2}, \frac{i_{-}}{2}, \tilde{\sigma}, \mu_{0}\right) D_{1}\left(\tilde{\sigma}, \mu_{0}\right)+\tau I_{2}\left(0, i_{-},\right.\right.\right. \\
& \left.\tilde{\sigma}, \mu_{0}\right) \tilde{D}_{1}\left(\frac{i_{-}}{2}, \tilde{\sigma}, \mu_{0}\right)+\tau \delta\left(i_{+}\right) D_{2}\left(\tilde{\sigma}, \mu_{0}\right) D_{1}\left(\tilde{\sigma}, \mu_{0}\right)+\tilde{I}_{2}\left(0, \frac{i_{+}}{2}, \frac{i_{+}}{2}, \tilde{\sigma}, \mu_{0}\right) D_{1}\left(\tilde{\sigma}, \mu_{0}\right)+I_{2}\left(0, i_{+},\right. \\
& \left.\tilde{\sigma}, \mu_{0}\right) \tilde{D}_{1}\left(\frac{i_{+}}{2}, \tilde{\sigma}, \mu_{0}\right)+\sum_{l=-\infty}^{\infty}\left[\tilde{I}_{2}\left(0, \frac{i_{-}}{2}+l, \frac{i_{-}}{2}-l, \tilde{\sigma}, \mu_{0}\right) \tilde{D}_{1}\left(l, \tilde{\sigma}, \mu_{0}\right)+\tau \tilde{I}_{2}\left(0, \frac{i_{+}}{2}+l, \frac{i_{+}}{2}-l, \tilde{\sigma}\right.\right. \\
& \left.\left.\left.\mu_{0}\right) \tilde{D}_{1}\left(l, \tilde{\sigma}, \mu_{0}\right)\right]\right]
\end{aligned}
$$

where

$$
D_{2}\left(\tilde{\sigma}, \mu_{0}\right)=\tilde{\sigma} \sum_{l=-\infty}^{\infty} \int \frac{d^{d-1} q}{\left[q^{2}+\tilde{\sigma}^{2} l^{2}+\mu_{0}^{2}\right]^{2}}
$$

The nontrivial two-loop graph of the two-point function can be obtained most easily by taking the four-point graph, making the identification $i_{1}=i_{3}=j$, introducing a new propagator with momentum $q_{2}$, mode $j$, performing a summation over $j$ and an integrating over $d^{d-1} q_{2}$. We find:

$$
\begin{aligned}
& \bigodot_{\left.\left.\tilde{\sigma}, \mu_{0}\right)\right]}=\left(\frac{N+2}{3}\right)\left[\left(\delta\left(i_{-}\right)+\tau \delta\left(i_{+}\right)\right) I_{3}\left(k, i_{1}, \tilde{\sigma}, \mu_{0}\right)+3 \tilde{I}_{3}\left(k, \frac{i_{+}}{2}, \frac{i_{-}}{2}, \tilde{\sigma}, \mu_{0}\right)+3 \tau \tilde{I}_{3}\left(k, \frac{i_{-}}{2}, \frac{i_{+}}{2},\right.\right.
\end{aligned}
$$


where,

$$
\begin{aligned}
& I_{3}\left(k, j, \tilde{\sigma}, \mu_{0}\right)=\tilde{\sigma}^{2} \sum_{j_{1}, j_{2}=-\infty}^{\infty} \int \frac{d^{d-1} q_{1} d^{d-1} q_{2}}{\left[q_{2}^{2}+\tilde{\sigma}^{2} j_{2}^{2}+\mu_{0}^{2}\right]\left[\left(q_{1}+q_{2}+k\right)^{2}+\tilde{\sigma}^{2}\left(j_{1}+j_{2}+j\right)^{2}+\mu_{0}^{2}\right]} \\
& \times \frac{1}{\left[q_{1}^{2}+\tilde{\sigma}^{2} j_{1}^{2}+\mu_{0}^{2}\right]}, \\
& \tilde{I}_{3}\left(k, i, j, \tilde{\sigma}, \mu_{0}\right)=\tilde{\sigma}^{2} \sum_{l=-\infty}^{\infty} \int \frac{d^{d-1} q_{1} d^{d-1} q_{2}}{\left[q_{1}^{2}+\tilde{\sigma}^{2} l^{2}+\mu_{0}^{2}\right]\left[q_{2}^{2}+\tilde{\sigma}^{2} i^{2}+\mu_{0}^{2}\right]} \\
& \times \quad \frac{1}{\left[\left(q_{1}+q_{2}+k\right)^{2}+\tilde{\sigma}^{2}(l+j)^{2}+\mu_{0}^{2}\right]} .
\end{aligned}
$$

The nontrivial three-loop graph contributing to the two-point function can be determined similarly. The complete result is:

$$
\begin{aligned}
& -=\frac{(N+2)(N+8)}{27}\left[\left(\delta\left(i_{-}\right)+\tau \delta\left(i_{+}\right)\right) I_{5}\left(k, i_{1}, \tilde{\sigma}, \mu_{0}\right)+\tilde{I}_{5}\left(k, \frac{i_{+}}{2}, \frac{i_{-}}{2}, \tilde{\sigma}, \mu_{0}\right)+\tau \tilde{I}_{5}\left(k, \frac{i_{-}}{2},\right.\right. \\
& \left.\frac{i_{+}}{2}, \tilde{\sigma}, \mu_{0}\right)+\hat{I}_{5}\left(k, \frac{i_{+}}{2}, \frac{i_{-}}{2}, i_{2}, \tilde{\sigma}, \mu_{0}\right)+\tau \hat{I}_{5}\left(k, \frac{i_{-}}{2}, \frac{i_{+}}{2}, i_{2}, \tilde{\sigma}, \mu_{0}\right)+2 \hat{I}_{5}\left(k, \frac{i_{+}}{2}, \frac{i_{-}}{2}, i_{1}, \tilde{\sigma}, \mu_{0}\right)+2 \tau \hat{I}_{5} \\
& \left(k, \frac{i_{-}}{2}, \frac{i_{+}}{2}, i_{1}, \tilde{\sigma}, \mu_{0}\right)+\check{I}_{5}\left(k, i_{1}, \frac{i_{-}}{2}, \frac{i_{+}}{2}, \tilde{\sigma}, \mu_{0}\right)+\tau \check{I}_{5}\left(k, i_{1}, \frac{i_{+}}{2}, \frac{i_{-}}{2}, \tilde{\sigma}, \mu_{0}\right)+\bar{I}_{5}\left(k, i_{1}, \frac{i_{+}}{2}, \frac{i_{-}}{2}, \tilde{\sigma}, \mu_{0}\right) \\
& \left.+\tau \bar{I}_{5}\left(k, i_{1}, \frac{i_{-}}{2}, \frac{i_{+}}{2}, \tilde{\sigma}, \mu_{0}\right)+\dot{I}_{5}\left(k, \frac{i_{+}}{2}, \frac{i_{-}}{2}, i_{1}, \tilde{\sigma}, \mu_{0}\right)+\tau \dot{I}_{5}\left(k, \frac{i_{-}}{2}, \frac{i_{+}}{2},-i_{2}, \tilde{\sigma}, \mu_{0}\right)\right]
\end{aligned}
$$

where the integrals above are defined by (the arguments $\left(k, l, m, n, \tilde{\sigma}, \mu_{0}\right)$ were suppressed in the integrals with three external quasi-momenta indices for sake of simplicity)

$$
\begin{aligned}
& I_{5}\left(k, i, \tilde{\sigma}, \mu_{0}\right)=\tilde{\sigma}^{3} \sum_{j_{1}, j_{2}, j_{3}=-\infty}^{\infty} \int \frac{d^{d-1} q_{1} d^{d-1} q_{2} d^{d-1} q_{3}}{\left[q_{1}^{2}+\tilde{\sigma}^{2} j_{1}^{2}+\mu_{0}^{2}\right]\left[q_{2}^{2}+\tilde{\sigma}^{2} j_{2}^{2}+\mu_{0}^{2}\right]\left[q_{3}^{2}+\tilde{\sigma}^{2} j_{3}^{2}+\mu_{0}^{2}\right]} \\
& \times \frac{1}{\left[\left(q_{1}+q_{2}+k\right)^{2}+\tilde{\sigma}^{2}\left(j_{1}+j_{2}+i\right)^{2}+\mu_{0}^{2}\right]\left[\left(q_{1}+q_{3}+k\right)^{2}+\tilde{\sigma}^{2}\left(j_{1}+j_{3}+i\right)^{2}+\mu_{0}^{2}\right]} \\
& \tilde{I}_{5}\left(k, i, j, \tilde{\sigma}, \mu_{0}\right)=\tilde{\sigma}^{3} \sum_{j_{1}, j_{2}=-\infty}^{\infty} \int \frac{d^{d-1} q_{1} d^{d-1} q_{2} d^{d-1} q_{3}}{\left[q_{1}^{2}+\tilde{\sigma}^{2} i^{2}+\mu_{0}^{2}\right]\left[q_{2}^{2}+\tilde{\sigma}^{2} j_{1}^{2}+\mu_{0}^{2}\right]\left[q_{3}^{2}+\tilde{\sigma}^{2} j_{2}^{2}+\mu_{0}^{2}\right]} \\
& \times \frac{1}{\left[\left(q_{1}+q_{2}+k\right)^{2}+\tilde{\sigma}^{2}\left(j_{1}+j\right)^{2}+\mu_{0}^{2}\right]\left[\left(q_{1}+q_{3}+k\right)^{2}+\tilde{\sigma}^{2}\left(j_{2}+j\right)^{2}+\mu_{0}^{2}\right]}, \\
& \hat{I}_{5}=\tilde{\sigma} \sum_{j=-\infty}^{\infty} \int \frac{d^{d-1} q \tilde{I}_{2}(q+k, l, j+m) I_{2}(q+k, j+n)}{q^{2}+\sigma^{2} j^{2}+\mu_{0}^{2}} \\
& \check{I}_{5}=\tilde{\sigma} \sum_{j_{1}, j_{2}=-\infty}^{\infty} \int \frac{d^{d-1} q \tilde{I}_{2}\left(q+k, j_{2}, j_{2}+j_{1}+l\right) \tilde{I}_{2}\left(q+k, j_{2}+m, j_{2}+j_{1}+n\right)}{q^{2}+j_{1}^{2}+\mu_{0}^{2}} \\
& \bar{I}_{5}=\tilde{\sigma} \sum_{j_{1}, j_{2}=-\infty}^{\infty} \int \frac{d^{d-1} q \tilde{I}_{2}\left(q+k, j_{2}, j_{2}+j_{1}+l\right) \tilde{I}_{2}\left(q+k, j_{1}+j_{2}+m, j_{2}+n\right)}{q^{2}+\sigma^{2} j_{1}^{2}+\mu_{0}^{2}} \\
& \dot{I}_{5}=\tilde{\sigma} \sum_{j_{1}, j_{2}=-\infty}^{\infty} \int \frac{d^{d-1} q \tilde{I}_{2}\left(q+k, j_{1}+l, m\right) \tilde{I}_{2}\left(q+k, j_{2}, 2 j_{2}+j_{1}+n\right)}{q^{2}+\sigma^{2} j_{1}^{2}+\mu_{0}^{2}}
\end{aligned}
$$


Let us turn our attention to the four-point vertex part. The simplest contribution at two-loops is the double bubble. Using the fusion of two one-loop diagrams of the four-point function results in the following unified expression $\left(I_{2}\left(k, i, \tilde{\sigma}, \mu_{0}\right) \equiv I_{2}(k, i), \tilde{I}_{2}\left(k, i, j, \tilde{\sigma}, \mu_{0}\right) \equiv\right.$ $\left.\tilde{I}_{2}(k, i, j)\right)$

$$
\begin{aligned}
& \mathcal{X}=\frac{\left(N^{2}+6 N+20\right)}{27} \tilde{\sigma}\left\{\left[\delta\left(i_{+}+\tilde{i}_{+}\right)+\tau \delta\left(i_{-}+\tilde{i}_{+}\right)+\delta\left(i_{+}-\tilde{i}_{+}\right)+\tau \delta\left(i_{-}-\tilde{i}_{+}\right)\right] I_{2}^{2}\left(k, \tilde{i}_{+}\right)\right. \\
& +\left[\tau \delta\left(i_{+}+\tilde{i}_{-}\right)+\delta\left(i_{-}+\tilde{i}_{-}\right)+\tau \delta\left(i_{+}-\tilde{i}_{-}\right)+\delta\left(i_{-}-\tilde{i}_{-}\right)\right] I_{2}^{2}\left(k, \tilde{i}_{-}\right)+2 \tau\left(I_{2}\left(k, \tilde{i}_{+}\right)+I_{2}\left(k, i_{+}\right)\right) \\
& \times \tilde{I}_{2}\left(k, \frac{i_{+}-\tilde{i}_{+}}{2}, \frac{i_{+}+\tilde{i}_{+}}{2}\right)+2 \tau\left(I_{2}\left(k, \tilde{i}_{-}\right)+I_{2}\left(k, i_{-}\right)\right) \tilde{I}_{2}\left(k, \frac{i_{-}-\tilde{i}_{-}}{2}, \frac{i_{-}+\tilde{i}_{-}}{2}\right)+2\left(I_{2}\left(k, \tilde{i}_{-}\right)\right. \\
& \left.+I_{2}\left(k, i_{+}\right)\right) \tilde{I}_{2}\left(k, \frac{i_{+}-\tilde{i}_{-}}{2}, \frac{i_{+}+\tilde{i}_{-}}{2}\right)+2\left(I_{2}\left(k, \tilde{i}_{+}\right)+I_{2}\left(k, i_{-}\right)\right) \tilde{I}_{2}\left(k, \frac{i_{-}-\tilde{i}_{+}}{2}, \frac{i_{-}+\tilde{i}_{+}}{2}\right) \\
& +2 \sum_{j=-\infty}^{\infty}\left(\tilde{I}_{2}\left(k, j, j+i_{+}\right)\left[\tilde{I}_{2}\left(k, j+\frac{i_{+}-\tilde{i}_{+}}{2}, j+\frac{i_{+}+\tilde{i}_{+}}{2}\right)+\tau \tilde{I}_{2}\left(k, j+\frac{i_{+}-\tilde{i}_{-}}{2}, j+\frac{i_{+}+\tilde{i}_{-}}{2}\right)\right]\right. \\
& \left.\left.+\tilde{I}_{2}\left(k, j, j+i_{-}\right)\left[\tilde{I}_{2}\left(k, j+\frac{i_{-}-\tilde{i}_{-}}{2}, j+\frac{i_{-}+\tilde{i}_{-}}{2}\right)+\tau \tilde{I}_{2}\left(k, j+\frac{i_{-}-\tilde{i}_{+}}{2}, j+\frac{i_{-}+\tilde{i}_{+}}{2}\right)\right]\right)\right\} .
\end{aligned}
$$

The important point which simplifies our task is to realize that the last terms like, for instance, $\sum_{j=-\infty}^{\infty} \tilde{I}_{2}\left(k, j, j+i_{+}\right) \tilde{I}_{2}\left(k, j+\frac{i_{+}-\tilde{i}_{+}}{2}, j+\frac{i_{+}+\tilde{i}_{+}}{2}\right)$ are $O\left(\epsilon^{0}\right)$ and do not contribute to the singular part of this diagram. These terms can be ignored.

Consider the evaluation of the nontrivial two-loop contribution of the four-point vertex function. Following the same line of thought we obtain $\left(P=k_{1}+k_{2}\right)$

$$
\begin{aligned}
& \qquad=\frac{(5 N+22)}{27} \tilde{\sigma}\left\{\left[\delta\left(i_{+}-\tilde{i}_{+}\right)+\tau \delta\left(i_{+}-\tilde{i}_{-}\right)\right] I_{4}\left(P, k_{3}, i_{+}, i_{3}\right)+\left[\delta\left(i_{-}-\tilde{i}_{-}\right)+\tau \delta\left(i_{-}-\tilde{i}_{+}\right)\right]\right. \\
& \times I_{4}\left(P, k_{3}, i_{-}, i_{3}\right)+\left[\delta\left(i_{+}+\tilde{i}_{+}\right)+\tau \delta\left(i_{+}+\tilde{i}_{-}\right)\right] I_{4}\left(P, k_{3}, i_{+},-i_{3}\right)+\left[\delta\left(i_{-}+\tilde{i}_{-}\right)+\tau \delta\left(i_{-}+\tilde{i}_{+}\right)\right] \times \\
& I_{4}\left(P, k_{3}, i_{-},-i_{3}\right)+\tilde{I}_{4}\left(P, k_{3}, \frac{i_{+}+\tilde{i}_{-}}{2}, \frac{i_{+}-\tilde{i}_{-}}{2}, \frac{i_{+}-\tilde{i}_{+}}{2}\right)+\tau \tilde{I}_{4}\left(P, k_{3}, \frac{i_{-}+\tilde{i}_{-}}{2}, \frac{i_{-}-\tilde{i}_{-}}{2}, \frac{i_{-}-\tilde{i}_{+}}{2}\right) \\
& +\tau \tilde{I}_{4}\left(P, k_{3}, \frac{i_{+}-\tilde{i}_{+}}{2}, \frac{i_{+}+\tilde{i}_{+}}{2}, \frac{i_{+}-\tilde{i}_{-}}{2}\right)+\tilde{I}_{4}\left(P, k_{3}, \frac{i_{-}-\tilde{i}_{+}}{2}, \frac{i_{-}+\tilde{i}_{+}}{2}, \frac{i_{-}-\tilde{i}_{-}}{2}\right)+\tau \tilde{I}_{4}\left(P, k_{3}, \frac{i_{+}}{2}\right. \\
& \left.+\frac{\tilde{i}_{+}}{2}, \frac{i_{+}-\tilde{i}_{+}}{2}, \frac{i_{+}-\tilde{i}_{-}}{2}\right)+\tilde{I}_{4}\left(P, k_{3}, \frac{i_{-}+\tilde{i}_{+}}{2}, \frac{i_{-}-\tilde{i}_{+}}{2}, \frac{i_{-}-\tilde{i}_{-}}{2}\right)+\tilde{I}_{4}\left(P, k_{3}, \frac{i_{+}-\tilde{i}_{-}}{2}, \frac{i_{+}+\tilde{i}_{-}}{2}, \frac{i_{+}}{2}\right. \\
& \left.+\frac{\tilde{i}_{+}}{2}\right)+\tau \tilde{I}_{4}\left(P, k_{3}, \frac{i_{-}-\tilde{i}_{-}}{2}, \frac{i_{-}+\tilde{i}_{-}}{2}, \frac{i_{-}+\tilde{i}_{+}}{2}\right)+2\left[\hat{I}_{4}\left(P, k_{3}, i_{+}, \frac{i_{+}+\tilde{i}_{-}}{2}, \frac{i_{+}-\tilde{i}_{+}}{2}\right)+\tau \hat{I}_{4}\left(P, k_{3},\right.\right. \\
& \left.i_{-}, \frac{i_{-}+\tilde{i}_{-}}{2}, \frac{i_{-}-\tilde{i}_{+}}{2}\right)+\tau \hat{I}_{4}\left(P, k_{3}, i_{+}, \frac{i_{+}-\tilde{i}_{+}}{2}, \frac{i_{+}+\tilde{i}_{-}}{2}\right)+\hat{I}_{4}\left(P, k_{3}, i_{-}, \frac{i_{-}-\tilde{i}_{+}}{2}, \frac{i_{-}+\tilde{i}_{-}}{2}\right)+ \\
& \hat{I}_{4}\left(P, k_{3}, i_{+}, \frac{i_{+}-\tilde{i}_{-}}{2}, \frac{i_{+}+\tilde{i}_{+}}{2}\right)+\tau \hat{I}_{4}\left(P, k_{3}, i_{-}, \frac{i_{-}-\tilde{i}_{-}}{2}, \frac{i_{-}+\tilde{i}_{+}}{2}\right)+\tau \hat{I}_{4}\left(P, k_{3}, i_{+}, \frac{i_{+}+\tilde{i}_{+}}{2}, \frac{i_{+}}{2}\right. \\
& \left.\left.\left.-\frac{\tilde{i}_{-}}{2}\right)+\hat{I}_{4}\left(P, k_{3}, i_{-}, \frac{i_{-}+\tilde{i}_{+}}{2}, \frac{i_{-}-\tilde{i}_{-}}{2}\right)\right]\right\},
\end{aligned}
$$

where the objects $I_{4}\left(k, k^{\prime}, i, j\right)\left(\equiv I_{4}\left(k, k^{\prime}, i, j ; \tilde{\sigma}, \mu_{0}\right)\right), \tilde{I}_{4}\left(k, k^{\prime}, i, j, l\right)\left(\equiv I_{4}\left(k, k^{\prime}, i, j, l ; \tilde{\sigma}, \mu_{0}\right)\right)$ 
and $\hat{I}_{4}\left(k, k^{\prime}, i, j, l\right)\left(\equiv I_{4}\left(k, k^{\prime}, i, j, l ; \tilde{\sigma}, \mu_{0}\right)\right)$ are defined, respectively, by

$$
\begin{aligned}
& I_{4}\left(k, k^{\prime}, i, j\right)=\tilde{\sigma}^{2} \sum_{l, m=-\infty}^{\infty} \int \frac{d^{d-1} q_{1} d^{d-1} q_{2}}{\left[q_{1}^{2}+\tilde{\sigma}^{2} l^{2}+\mu_{0}^{2}\right]\left[\left(q_{1}-k\right)^{2}+\tilde{\sigma}^{2}(l-i)^{2}+\mu_{0}^{2}\right]\left[q_{2}^{2}+\tilde{\sigma}^{2} m^{2}+\mu_{0}^{2}\right]} \\
& \times \frac{1}{\left[\left(q_{1}-q_{2}+k^{\prime}\right)^{2}+\tilde{\sigma}^{2}(l-m-j)^{2}+\mu_{0}^{2}\right]}, \\
& \tilde{I}_{4}\left(k, k^{\prime}, i, j, l\right)=\tilde{\sigma}^{2} \sum_{m=-\infty}^{\infty} \int \frac{d^{d-1} q_{1} d^{d-1} q_{2}}{\left[q_{1}^{2}+\tilde{\sigma}^{2} i^{2}+\mu_{0}^{2}\right]\left[\left(q_{1}-k\right)^{2}+\tilde{\sigma}^{2} j^{2}+\mu_{0}^{2}\right]\left[q_{2}^{2}+\tilde{\sigma}^{2} m^{2}+\mu_{0}^{2}\right]} \\
& \times \frac{1}{\left[\left(q_{1}-q_{2}+k^{\prime}\right)^{2}+\tilde{\sigma}^{2}(l-m)^{2}+\mu_{0}^{2}\right]}, \\
& \hat{I}_{4}\left(k, k^{\prime}, i, j, l\right)=\tilde{\sigma}^{2} \sum_{m=-\infty}^{\infty} \int \frac{d^{d-1} q_{1} d^{d-1} q_{2}}{\left[q_{1}^{2}+\tilde{\sigma}^{2} m^{2}+\mu_{0}^{2}\right]\left[\left(q_{1}-k\right)^{2}+\tilde{\sigma}^{2}(m-i)^{2}+\mu_{0}^{2}\right]\left[q_{2}^{2}+\tilde{\sigma}^{2} j^{2}+\mu_{0}^{2}\right]} \\
& \times \frac{1}{\left[\left(q_{1}-q_{2}+k^{\prime}\right)^{2}+\tilde{\sigma}^{2}(l-m)^{2}+\mu_{0}^{2}\right]} .
\end{aligned}
$$

Note that the pole in $\epsilon$ coming from the computation of the summation is absent in the integral $\hat{I}_{4}$. Since we are only interested in the singular part of the above diagram, we can simply neglect the contribution coming from this integral.

We are now left with the two-loop diagrams of the vertex part $\Gamma^{(2,1)}\left(k_{1}, k_{2} ; Q, i_{1}, i_{2}, j, \mu_{0}, \tilde{\sigma}\right)$. Sticking to the method above described using a similar simplifying notation for the integrals by omitting the arguments $\left(\mu_{0}, \tilde{\sigma}\right)$, whereas performing the identifications $\left(k_{i}\right) \equiv\left(k_{1}, k_{2} ; Q\right), k=k_{1}+k_{2}$, it is not difficult to find the following expressions $\left(i_{ \pm}=i_{1} \pm i_{2}\right)$ :

$$
\begin{aligned}
& \mathcal{C}^{\mathrm{mun}}\left(k_{i}\right)=\frac{\tau}{2} \frac{(N+2)^{2}}{108}\left[\left(\delta\left(i_{+}+j\right)+\delta\left(i_{+}-j\right)+\tau \delta\left(i_{-}+j\right)+\tau \delta\left(i_{-}+j\right)\right) I_{2}^{2}(k, j)\right. \\
& +2 \tau \tilde{I}_{2}\left(k, \frac{j+i_{+}}{2}, \frac{j-i_{+}}{2}\right)\left[I_{2}(k, j)+I_{2}\left(k, i_{+}\right)\right]++2 \tilde{I}_{2}\left(k, \frac{j+i_{-}}{2}, \frac{j-i_{-}}{2}\right)\left[I_{2}(k, j)+I_{2}\left(k, i_{-}\right)\right] \\
& +2 \sum_{l=-\infty}^{\infty}\left(\tilde{I}_{2}\left(k, \frac{j-i_{+}-2 l}{2}, \frac{j+i_{+}+2 l}{2}\right) \tilde{I}_{2}\left(k, l, l+i_{+}\right)+\tilde{I}_{2}\left(k, \frac{j-i_{-}-2 l}{2}, \frac{j+i_{-}+2 l}{2}\right)\right. \\
& \left.\left.\times \tilde{I}_{2}\left(k, l, l+i_{-}\right)\right)\right] \\
& \biguplus_{i}\left(k_{i}\right)=\frac{\tau}{2} \frac{(N+2)}{36}\left\{\left(\delta\left(i_{+}+j\right)+\delta\left(i_{+}-j\right)\right) I_{4}\left(k, Q, i_{+}, i_{2}\right)\right)+\tau\left(\delta\left(i_{-}+j\right)+\delta\left(i_{-}-j\right)\right) \\
& \times I_{4}\left(k, Q, i_{-}, i_{2}\right)+\tilde{I}_{4}\left(k, Q, \frac{j-i_{-}}{2}, \frac{j+i_{-}}{2}, \frac{j-i_{+}}{2}\right)+\tau \tilde{I}_{4}\left(k, Q, \frac{j+i_{+}}{2}, \frac{j-i_{+}}{2}, \frac{j+i_{-}}{2}\right)+\tilde{I}_{4}(k, Q, \\
& \left.\frac{j+i_{-}}{2}, \frac{j-i_{-}}{2}, \frac{j+i_{+}}{2}\right)+\tau \tilde{I}_{4}\left(k, Q, \frac{j-i_{+}}{2}, \frac{j+i_{+}}{2}, \frac{j-i_{-}}{2}\right)+2 \tau \hat{I}_{4}\left(k, Q, j, \frac{j-i_{+}}{2}, \frac{j-i_{-}}{2}\right) \\
& \left.+2 \hat{I}_{4}\left(k, Q, j, \frac{j+i_{-}}{2}, \frac{j+i_{+}}{2}\right)+2 \tau \hat{I}_{4}\left(k, Q, j, \frac{j+i_{+}}{2}, \frac{j+i_{-}}{2}\right)+2 \hat{I}_{4}\left(k, Q, j, \frac{j-i_{-}}{2}, \frac{j-i_{+}}{2}\right)\right\}
\end{aligned}
$$


We have now a set of integrals which are very similar to those coming from periodic boundary conditions (see Ref. [22]). From our discussion, getting the expression of the diagrams is the laborious part of the method. Since we have already discussed the periodic integrals, only minor modifications are necessary to perform the computations aimed as we shall see next.

\section{MASSIVE FIELDS FOR $D B C$ AND $N B C$ IN THE EXPONENTIAL REPRE- SENTATION}

The vertex parts depend on the boundary condition, but we will suppress that dependence in what follows. By now it is rather clear that our unified description in the last section leaves no ambiguity to define the renormalization algorithm simultaneously for both boundary conditions.

Before discussing explicitly the renormalization algorithm, it is interesting to restrict ourselves to the minimal number of diagrams to perform our computation of the critical exponents. The argument for the massive theory regarding infinite systems appeared recently [25]. We will summarize the steps here and use the results directly. Start by defining the three-loop bare mass $\tilde{\mu}_{0}$ in terms of the the tree-level bare mass as $\tilde{\Gamma}^{(2)}\left(k=0, j, \tilde{\sigma}, \mu_{0}, \lambda_{0}\right) \equiv$ $\tilde{\mu}_{0}^{2}=\Gamma^{(2)}\left(k=0, j, \tilde{\sigma}, \mu_{0}, \lambda_{0}\right)-\tilde{\sigma}^{2} j^{2}$. Then, inverting this equation to get the tree-level $\mu_{0}$ in terms of the three-loop bare mass $\tilde{\mu}_{0}$ and all the diagrams computed at zero external momenta has the virtue of eliminating all tadpole diagrams for they do not depend on the external momentum. Next, expanding $\mu_{0}=\mu_{0}\left(\tilde{\mu}_{0}, \lambda_{0}\right)$ in each primitively divergent vertex part at first order in $\lambda_{0}$ eliminates all the remaining graphs containing one-loop mass insertions. Here is one of the most nontrivial results and we are going to discuss this topic carefully in what follows.

Recall that the two-point function always includes a factor $S_{i_{1} i_{2}}=\delta\left(i_{1}-i_{2}\right)$ in arbitrary loop diagrams. The set of steps just described permits one to write the tree-level bare mass in terms of the three-loop bare mass up to $O\left(\lambda_{0}\right)$ through

$$
\mu_{0}^{2}=\tilde{\mu}_{0}^{2}-\frac{\lambda_{0}}{2} \bigcirc
$$

Notice that when we perform this expansion in any integral, a complication occurs: the tadpole graph has finite size (kind of "internal symmetry") indices, say $l, m$, which will be 
attached to a propagator of a certain internal line of an arbitrary graph. So these indices should be contracted with the set of indices taking place naturally in the diagram. Consider the two-loop diagram of the four-point function with a "mass insertion":

$$
\begin{aligned}
& \text { 久. }=\frac{(N+8)(N+2)}{27} \sum_{j_{1}, j_{2}, j_{3}, j_{4} \geq 0}^{\infty} \tilde{S}_{i_{1} i_{2} j_{1} j_{2}}^{(\tau)} \tilde{S}_{j_{1} j_{3} j_{4} j_{4}}^{(\tau)} \tilde{S}_{j_{2} j_{3} i_{3} i_{4}}^{(\tau)} \int d^{d-1} q_{1} d^{d-1} q_{2} G_{0}\left(q_{1}+k, j_{1}\right) \\
& G_{0}\left(q_{1}+k, j_{3}\right) G_{0}\left(q_{1}, j_{2}\right) G_{0}\left(q_{2}, j_{4}\right) .
\end{aligned}
$$

(The condensed nomenclature $\tilde{S}_{i_{1} i_{2} j_{1} j_{2}}^{(\tau)}=\tilde{\sigma}\left[i_{1} i_{2} j_{1} j_{2}\right]_{\tau}$ is sometimes useful.) In this expression we can set $\mu_{0}=\tilde{\mu}_{0}$ since the correction will produce three-loop terms which are not going to be useful to our analysis of the four-point function up to two-loop order and is going to be neglected.

Let us take a look in Eq. (19). There the propagator is defined as $G_{0}(q, j) \equiv G_{0}\left(q, j, \mu_{0}\right)$. When we replace the mass $\mu_{0}$ by $\tilde{\mu}_{0}$ into the propagator, the left of the graph carries index $j$ from the original propagator before the insertion. Due to the internal character of the propagator which carries finite-size symmetry index, the coupling of the left hand side with the inserted mass tadpole is implemented as follows: the index $j$ must be free in the resulting expression and all the the other indices appearing there should be contracted with summations. This is equivalent to the following simple rule

$$
\begin{aligned}
& G_{0}\left(q, j, \mu_{0}\right)=G_{0}\left(q, j, \tilde{\mu}_{0}\right)\left[1-\sum_{l \geq 0}^{\infty} \frac{\lambda_{0}}{2\left[q^{2}+\tilde{\sigma}^{2} l^{2}+\tilde{\mu}_{0}^{2}\right]}[\bigcirc]_{j l}\right]^{-1} \\
& =G_{0}\left(q, j, \tilde{\mu}_{0}\right)+\frac{\lambda_{0}}{2} \sum_{l \geq 0}^{\infty} G_{0}\left(q, j, \tilde{\mu}_{0}\right) G_{0}\left(q, l, \tilde{\mu}_{0}\right)[\bigcirc]_{j l} .
\end{aligned}
$$

Now, replace this result in Eq. (19). The one-loop diagram has two propagators which contribute the same amount to the correction due to the mass insertion (after some redefinitions). Upon substitution of Eq. (8) for the tadpole, we find

$$
\begin{aligned}
& \Upsilon \mathcal{X}=\frac{(N+8)}{9} \sum_{l_{1}, l_{2} \geq 0}^{\infty} \tilde{S}_{i_{1} i_{2} l_{1} l_{2}}^{(\tau)} \tilde{S}_{l_{1} l_{2} i_{3} i_{4}}^{(\tau)} \int d^{d-1} q G_{0}\left(q+k, l_{1}, \tilde{\mu}_{0}\right) G_{0}\left(q, l_{2}, \tilde{\mu}_{0}\right) \\
& +\lambda_{0} \frac{(N+8)(N+2)}{27} \sum_{j_{1}, j_{2}, j_{3}, j_{4} \geq 0}^{\infty} \tilde{S}_{i_{1} i_{2} j_{1} j_{2}}^{(\tau)} \tilde{S}_{j_{1} j_{3} j_{4} j_{4}}^{(\tau)} \tilde{S}_{j_{2} j_{3} i_{3} i_{4}}^{(\tau)} \int d^{d-1} q_{1} d^{d-1} q_{2} G_{0}\left(q_{1}+k, j_{1}, \tilde{\mu}_{0}\right) \\
& G_{0}\left(q_{1}+k, j_{3}, \tilde{\mu}_{0}\right) G_{0}\left(q_{1}, j_{2}, \tilde{\mu}_{0}\right) G_{0}\left(q_{2}, j_{4}, \tilde{\mu}_{0}\right) .
\end{aligned}
$$

Then, we conclude that the two-loop correction term due to the expansion of the internal propagators in the one-loop diagram of the four-point function produces the mass insertion 
whose value is equal exactly to that from Eq. (63). This statement is equivalent to the important graphical identity:

$$
\Upsilon \times\left.\right|_{\mu_{0}}=\Upsilon \times\left.\right|_{\tilde{\mu}_{0}}+\lambda_{0} 久 \times\left.\right|_{\tilde{\mu}_{0}} .
$$

By following the same prescription, all the primitively divergent vertex parts with mass (tadpole) insertion are eliminated. For instance, the reader can check from what we have been discussing that the following diagrammatic identity also holds

$$
\left.\bigodot\right|_{\mu_{0}}=\left.\bigodot\right|_{\tilde{\mu}_{0}}+\frac{3 \lambda_{0}}{2} \bigodot_{\tilde{\mu}_{0}}
$$

It is easy to conclude that similar diagrammatic identities are valid for the composite operator $\Gamma^{(2,1)}$ due to its similarity with $\Gamma^{(4)}$. Despite the apparently complicated tensorial structure of this finite-size field-theoretic formulation, the mass insertions are cancelled for arbitrary external quasi-momenta modes as shown above in a simple manner. This cancellation takes place since different orders in $\lambda_{0}$ in the perturbative expression have different signs, and the combinatorial factors coming from the mass insertion corrections in each diagram exactly matches those from the perturbation expansion of the original diagram (one power of $\lambda_{0}$ higher) in $\mu_{0}$. This suffices to prove the claim explicitly at the perturbative order considered in the present paper. See [25] for more details.

The resulting vertex parts now depend exclusively on $\tilde{\mu}_{0}, \lambda_{0}$. Their diagrammatic expansion in terms of the reduced number of diagrams are:

$$
\begin{aligned}
& \tilde{\Gamma}^{(2)}\left(k, j, \tilde{\sigma}, \tilde{\mu}_{0}, \lambda_{0}\right)=k^{2}+\tilde{\mu}_{0}^{2}-\frac{\lambda_{0}^{2}}{6}\left(\left.\bigodot\right|_{\tilde{\mu}_{0}}-\left.\bigodot\right|_{k=0, \tilde{\mu}_{0}}\right)+\frac{\lambda_{0}^{3}}{4}\left(\bigodot \begin{array}{|}
\tilde{\mu}_{0} \\
\mid
\end{array}\right. \\
& \left.-\left.\bigodot\right|_{k=0, \tilde{\mu}_{0}}\right) \\
& \Gamma_{i_{1} i_{2} i_{3} i_{4}}^{(4)}\left(k_{i}, \tilde{\sigma}, \mu_{0}, \lambda_{0}\right)=\lambda_{0} \tilde{S}_{i_{1} i_{2} i_{3} i_{4}}-\frac{\lambda_{0}^{2}}{2}\left([久]_{\tilde{\mu}_{0}}\left(k_{1}+k_{2}\right)+2 \text { perms. }\right)+\frac{\lambda_{0}^{3}}{2}\left\{(\smile \bigcirc]_{\tilde{\mu}_{0}}\right. \\
& \left.\left.\left(k_{i}\right)+5 \text { perms. }\right)+\frac{1}{2}\left([\curlywedge \bigcirc]_{\tilde{\mu}_{0}}\left(k_{1}+k_{2}\right)+2 \text { perms. }\right)\right\} \text {, } \\
& \Gamma_{i_{1}, i_{2}, j}^{(2,1)}\left(k_{1}, k_{2} ; Q_{3}, \tilde{\sigma}, \mu_{0}, \lambda_{0}\right)=\tau \Gamma_{i_{1}, i_{2}, j=0}^{(2,1)}+\Gamma_{i_{1}, i_{2}, j=i_{1}+i_{2}}^{(2,1)} \\
& \Gamma_{i_{1}, i_{2}, j}^{(2,1)}\left(k_{1}, k_{2} ; Q_{3}, \tilde{\sigma}, \mu_{0}, \lambda_{0}\right)=\sum_{1--\sum_{-2}}^{3}-3 \lambda_{0}[\text { 妾 }]_{\tilde{\mu}_{0}}\left(k_{1}+k_{2} ; Q_{3}\right)+3 \lambda_{0}^{2}[\underbrace{\text { mun }}_{\tilde{\mu}_{0}}]_{1}\left(k_{1}+k_{2} ; Q_{3}\right) \\
& +6 \lambda_{0}^{2}[\circlearrowleft]_{\tilde{\mu}_{0}}\left(k_{1}, k_{2} ; Q_{3}\right) \text {. }
\end{aligned}
$$

We choose not to display explicitly the tensor $S_{i_{1} i_{2}}$ for the vertex $\Gamma^{(2)}$ since after using the value of this tensor, the two-point function depends upon only one external quasimomentum (mode). Note that Eq. (68c) takes into account that each diagram appears in 
the combination mentioned before in Eq. (38). We appplied a similar simplifying notation for the vertex part $\Gamma_{i_{1} i_{2} j}^{(2,1)}$ and choose not to show the dependence on the tensor $\hat{S}_{i_{1}, i_{2}, j}$ in the zeroth order diagram because of that implicit combination. (We could have done the same to the vertex part $\Gamma_{i_{1}, i_{2}, i_{3}, i_{4}}^{(4)}$ provided we include the zero order graph in the diagrammatic expansion.) We omit hereafter the lower indices ("internal" finite-size modes) whenever referring to an arbitrary vertex part but keep then on the argument of that vertex.

The vertex part $\tilde{\Gamma}^{(2)}\left(k, j, \tilde{\sigma}, \tilde{\mu}_{0}, \lambda_{0}\right) \equiv \Gamma^{(2)}\left(k, j, \tilde{\sigma}, \tilde{\mu}_{0}, \lambda_{0}\right)-\tilde{\sigma}^{2} j^{2}$ has the advantage of not possessing the tree-level term $\tilde{\sigma}^{2} j^{2}$ and looks like the two-point vertex part from the bulk case. It has a logarithmic divergence as well as $\Gamma^{(4)}\left(k_{i}, j_{i}, \tilde{\sigma}, \tilde{\mu}_{0}, \lambda_{0}\right)$. In our specific case of a given order in the coupling constant expansion, we want to go up to threeloop order in the expansion of $\tilde{\Gamma}^{(2)}$. Define the renormalized quantity $\tilde{\Gamma}_{R}^{(2)}\left(k, j, \tilde{\sigma}, \tilde{\mu}_{0}, \lambda_{0}\right)=$ $Z_{\phi} \tilde{\Gamma}^{(2)}\left(k, j, \tilde{\sigma}, \tilde{\mu}_{0}, \lambda_{0}\right)$. The introduction of the normalization function $Z_{\phi}$ produces the cancellation of the logarithmic divergence aforementioned. After that, we define a renormalized finite mass at three-loop order as $\mu^{2}=Z_{\phi} \tilde{\mu}_{0}^{2}$. On the other hand, the four-point vertex part starts proportional to the bare coupling constant, so we can define a renormalized coupling constant up to two-loop level by writing $g=Z_{\phi}^{2} \lambda_{0}$. First, write $Z_{\phi}=1+g z_{1}+g^{2} z_{2}+g^{3} z_{3}+\ldots$ (where $z_{n}$ are divergent quantities). From the absence of linear terms in the coupling constant perturbative expansion of $\tilde{\Gamma}_{R}^{(2)}$, we immediately obtain $z_{1}=0$. At third order, we can express it entirely in terms of renormalized quantities, $\tilde{\Gamma}_{R}^{(2)}(k, j, \tilde{\sigma}, \mu, g) \equiv \tilde{\Gamma}_{R}^{(2)}\left(k, j, \tilde{\sigma}, \tilde{\mu}_{0}, \lambda_{0}\right)$. The same happens to the other vertex part, since up to two-loop order the renormalized object defined by $\Gamma_{R}^{(4)}\left(k_{i}, j_{i}, \tilde{\sigma}, \mu, g\right)=Z_{\phi}^{2} \Gamma^{(4)}\left(k, j_{i}, \tilde{\sigma}, \tilde{\mu}_{0}, \lambda_{0}\right)$ is automatically given in terms only of renormalized mass and coupling constant, as the reader can easily verify. The same argument carries through to the renormalized composed vertex part obtained from the logarithmic divergent bare one via $\Gamma_{R}^{(2,1)}(k, j, \tilde{\sigma}, \mu, g)=Z_{\phi^{2}} Z_{\phi} \Gamma^{(2)}\left(k, j, \tilde{\sigma}, \tilde{\mu}_{0}, \lambda_{0}\right)$. It requires another normalization function $Z_{\phi^{2}}$ and no longer depends on bare quantities at this order.

In general, we can go to arbitrary loop order and get rid of all the bare quantities in the definition of generic vertex parts which are renormalized multiplicatively. Multiplicative renormalizability amounts to say that an arbitrary vertex part $\Gamma^{(L, M)}((L, M) \neq(0,2))$ including composite operators can be renormalized through the functions $Z_{\phi}^{(\tau)}, Z_{\phi^{2}}^{(\tau)}$ such that the vertex parts defined by

$$
\Gamma_{R}^{(L, M)}\left(p_{n}, i_{n}, Q_{n^{\prime}}, j_{n^{\prime}}, g, \mu\right)=\left(Z_{\phi}^{(\tau)}\right)^{\frac{M}{2}}\left(Z_{\phi^{2}}^{(\tau)}\right)^{L} \Gamma^{(L, M)}\left(p_{n}, i_{n}, Q_{n^{\prime}}, j_{n^{\prime}}, \lambda_{0}, \tilde{\mu}_{0}, \Lambda\right)
$$


are automatically finite. (In the argument above $\Gamma_{R}^{(L, M)} \equiv \tilde{\Gamma}_{R}^{(L, M)}$ for $(L, M)=(2,0)$.) Here, $g, \mu$ and $\Lambda$ are the renormalized coupling constant, mass and cutoff, respectively. The argument works well for all kinds of regulators, which are used to express infinite (divergent) quantities in terms of functions of these parameters (regulators). We employ the cutoff when deriving differential equations satisfied by renormalized vertex parts. From the explicit operational viewpoint, however, we shall focus here in dimensional regularization, so that divergences are written in terms of poles in $\epsilon=4-d\left(\propto \epsilon^{-l}\right.$, where $l$ is an integer positive number, usually the number of loops), where $d$ is the space dimension of the system. The symbol $n$ labels the external momenta $p_{n}$ as well as the mode of the external quasi-momenta $i_{n}$ associated to the external legs $(n=1, \ldots, L)$ of the fields. The label $n^{\prime}$ is connected to the external momenta $Q_{n^{\prime}}$ and the mode $j_{n^{\prime}}$ of the quasi-momentum corresponding to the external legs $\left(n^{\prime}=1, \ldots, M\right)$ of the composite fields ( $\phi^{2}$ insertions $)$ in an arbitrary $1 P I$ diagram.

The normalization conditions on the primitively divergent vertex parts for the massive theory are chosen at zero external momenta and nonzero quasi-momenta. From the structure of the diagrams, it is obvious that the condition on the external momenta are not sufficient to simplify our task. In fact, there are other renormalization schemes, for instance minimal subtraction, which do not require any fixation of the external momenta. In order to give a simpler prescription useful for all other renormalization methods required from an $a b$ initio formulation, we choose the external quasi-momenta to be set at especial values. The symmetry point here is defined at external quasi-momenta from the external legs chosen to be equal and set to the value $i$ (arbitrary nonvanishing positive integer). The finiteness of the theory can be achieved through the conditions

$$
\begin{aligned}
& \tilde{\Gamma}_{R}^{(2)}(k=0, i, g, \mu)=\mu^{2}, \\
& \left.\frac{\partial \tilde{\Gamma}_{R}^{(2)}(k, i, g, \mu)}{\partial k^{2}}\right|_{k^{2}=0}=1, \\
& \left.\Gamma_{R}^{(4)}\left(k_{l}=0, i_{l}=i, g, \mu\right) \equiv \Gamma_{R}^{(4)}\right|_{S P}=3 \tilde{\sigma} g, \\
& \left.\Gamma_{R}^{(2,1)}\left(k=0, i_{1}=i_{2}=i, Q=0, j, g, \mu\right) \equiv \Gamma_{R}^{(2,1)}\right|_{\overline{S P}}=\frac{3 \tau}{2} .
\end{aligned}
$$

We can now discuss the renormalization group invariance of arbitrary renormalized vertex functions. At the critical dimension $d=4$ utilize the cutoff as the regulator. The infinite cutoff limit in the integrals appearing in each vertex part multiplicatively renormalized is 
taken solely after the renormalization prescription is established. Apply the derivative $\frac{\partial}{\partial \tilde{\mu}_{0}^{2}}$ over the bare vertex part $\Gamma^{(N, M)}((N, M) \neq(0,2))$ at fixed $\lambda_{0}, \Lambda$ in order to obtain the vertex function $\Gamma^{(N, M+1)}\left(p_{n}, i_{n}, Q_{n^{\prime}}, i_{n^{\prime}}^{\prime} ; 0 ; \lambda_{0}, \tilde{\mu}_{0}, \Lambda\right)$ at zero inserted momentum. Next, rewrite the remaining bare vertex parts in terms of the renormalized ones. After similar manipulations performed in the case of periodic and antiperiodic boundary conditions in [22] but utilizing the above normalization conditions, one learns that the renormalization group invariance of the renormalized vertex parts in different renormalized mass scales is substantiated in the following form of the Callan-Symanzik equation:

$$
\begin{aligned}
& \left(\mu \frac{\partial}{\partial \mu}+\beta^{(\tau)} \frac{\partial}{\partial g}-\frac{N}{2} \gamma_{\phi}^{(\tau)}+M \gamma_{\phi^{2}}^{(\tau)}\right) \Gamma_{R}^{(N, M)}\left(p_{l}, i_{l}, Q_{l}, i_{l}^{\prime}, g, \mu\right)= \\
& \mu^{2}\left(2-\gamma_{\phi}^{(\tau)}\right) \Gamma_{R}^{(N, M+1)}\left(p_{l}, i_{l}, Q_{l}, i_{l}^{\prime} ; 0, g, \mu\right),
\end{aligned}
$$

where $\beta^{(\tau)}(\mu, g)=\mu \frac{\partial g}{\partial \mu}, \gamma_{\phi}^{(\tau)}=\mu \frac{\partial \ln Z_{\phi}^{(\tau)}}{\partial \mu}$ and $\gamma_{\phi^{2}}^{(\tau)}=-\mu \frac{\partial \ln Z_{\phi^{2}}^{(\tau)}}{\partial \mu}$. Even for $i \neq 0$ in the present unified framework, this form resembles very much the situation for $P B C$.

When the field is not at the critical dimension, the annoying dimensionful aspect of the coupling constant can be circumvented by writing the bare (renormalized) coupling constant in terms of a genuine dimensionless bare (renormalized) coupling constant $u_{0}(u)$ as $\lambda_{0}=\mu^{\epsilon} u_{0}\left(g=\mu^{\epsilon} u\right)$. The flow function $\beta^{(\tau)}(\mu, g)$ can be written in terms Gell-Mann-Low function defined by $[\beta(g, \mu)]_{G L}=-\epsilon g+\beta(g, \mu)$. Using the Gell-Mann-Low function into the $C S$ equation, along with the dimensionful quantities defined in terms of dimensionless amounts, we find that $[\beta(g, \mu)]_{G L} \frac{\partial}{\partial g}=\beta(u) \frac{\partial}{\partial u}$. It turns out that resulting renormalizationgroup picture involves only the dimensionless renormalized coupling constant and possesses a well defined scaling limit [29, 30]. The Callan-Symanzik equation now reads

$$
\begin{aligned}
& \left(\mu \frac{\partial}{\partial \mu}+\beta(u) \frac{\partial}{\partial u}-\frac{L}{2} \gamma_{\phi}^{(\tau)}+M \gamma_{\phi^{2}}^{(\tau)}\right) \Gamma_{R}^{(L, M)}\left(p_{n}, i_{n}, Q_{n}, i_{n^{\prime}}^{\prime}, u, \mu\right)= \\
& \mu^{2}\left(2-\gamma_{\phi}^{(\tau)}\right) \Gamma_{R}^{(L, M+1)}\left(p_{n}, i_{n}, Q_{n^{\prime}}, i_{n^{\prime}}^{\prime} ; 0, u, \mu\right),
\end{aligned}
$$

where

$$
\begin{aligned}
& \beta^{(\tau)}(u)=-\epsilon\left(\frac{\partial \ln u_{0}^{(\tau)}}{\partial u}\right), \\
& \gamma_{\phi}^{(\tau)}(u)=\beta^{(\tau)}(u)\left(\frac{\partial \ln Z_{\phi}^{(\tau)}}{\partial u}\right), \\
& \gamma_{\phi^{2}}^{(\tau)}(u)=\beta^{(\tau)}(u)\left(\frac{\partial \ln Z_{\phi^{2}}^{(\tau)}}{\partial u}\right) .
\end{aligned}
$$


Another function that will be useful to our purposes and utilizes the definition $\bar{Z}_{\phi^{2}}^{(\tau)}=$ $Z_{\phi^{2}}^{(\tau)} Z_{\phi}^{(\tau)}$ is written as

$$
\bar{\gamma}_{\phi^{2}}^{(\tau)}=\beta^{(\tau)}\left(\frac{\partial \ln \bar{Z}_{\phi^{2}}^{(\tau)}}{\partial u}\right)
$$

The solution of the Callan-Symanzik equation has already been described in the ultraviolet scaling regime. The right-hand side becomes negligible at the scaling limit, $\frac{p}{\mu} \rightarrow \infty$ [27, 28], showing that the solution admits a ultraviolet fixed point. We will just make use of these results here and prove that the definitions above are sufficient to our computation of the fixed point and its aftermath in the evaluation of critical indices.

Let us start the discussion of the asymptotic limits for both boundary conditions. The basic objects to be computed are the one-loop integrals $I_{2}(k, i, \tilde{\sigma}, \mu)$ and $\tilde{I}_{2}(k, i, j, \tilde{\sigma}, \mu)$ which belong to the one-loop graph of the four-point function. The resemblence of these integrals for $D B C, N B C, A B C$ and $P B C$ will permit a more economical approach to this topic and the reader is advised to consult Ref. [22] to grasp many details. We prefer to omit them herein.

The integral $I_{2}(k, i, \tilde{\sigma}, \mu)$ is identical in the form to $I_{2}^{(\tau)}(k, i, \sigma, \mu)$ for periodic boundary condition ( $\tau=0$ in Eq. (10) from [22]). First, we factor out the mass $\mu$ from the integral, rescale all momenta and define $\tilde{r}=\frac{\tilde{\sigma}}{\mu}$. Second, we introduce a Feynman parameter and solve the integral over the momenta. Third, we perform the summation using the representation from Ref. [24]. Fourth, transform the argument of the resulting (factorial) $\Gamma$ function from $(d-1)$ to $d$. Then, divide the integral by the area of the unit sphere at $d$ dimensions. These sets of steps lead to the following result (see also Appendix A)

$$
\begin{aligned}
& I_{2}(k, i ; \tilde{r}) \equiv \frac{I_{2}(k, i ; \tilde{\sigma}, \mu)}{S_{d}}=\frac{\mu^{-\epsilon}}{\epsilon}\left(\left(1-\frac{\epsilon}{2}\right) \int_{0}^{1} d x\left[x(1-x)\left(k^{2}+\tilde{r}^{2} i^{2}\right)+1\right]^{-\frac{\epsilon}{2}}\right. \\
& \left.\quad+\frac{\epsilon}{2} \Gamma\left(2-\frac{\epsilon}{2}\right) F_{\frac{\epsilon}{2}}(k, i ; \tilde{r})\right),
\end{aligned}
$$

where

$$
\begin{aligned}
& \tilde{F}_{\alpha}(k, i ; \tilde{r})=\tilde{r}^{-2 \alpha} \int_{0}^{1} d x f_{\frac{1}{2}+\alpha}(x i, h(k, i, \tilde{r})) \\
& f_{\alpha}(a, b)=4 \sum_{m=1}^{\infty} \cos (2 \pi m a)\left(\frac{\pi m}{b}\right)^{\alpha-\frac{1}{2}} K_{\alpha-\frac{1}{2}}(2 \pi m b), \\
& h(k, i, \tilde{r})=\tilde{r}^{-1} \sqrt{x(1-x)\left(k^{2}+\tilde{r}^{2} i^{2}\right)+1}
\end{aligned}
$$

where $K_{\nu}(x)$ is the modified Bessel function of the second kind. 
Using a similar chain of reasoning, it is not difficult to find that the other integral required reads:

$$
\tilde{I}_{2}(k, i, j ; \tilde{r}) \equiv \frac{\tilde{I}_{2}(k, i, j ; \tilde{\sigma}, \mu)}{S_{d}}=\frac{\tilde{r} \mu^{-\epsilon}}{2} \int_{0}^{1} d x\left(\tilde{r}^{2}\left[x i^{2}+(1-x) j^{2}\right]+k^{2} x(1-x)+1\right)^{-\frac{1}{2}}
$$

Recall that in order to compute the one-loop diagram, owing to our use of normalization conditions in this massive framework, we need the previous integrals evaluated at vanishing external momenta $(k=0)$. Not only this: specifically, we need $I_{2}(0,2 i, \tilde{r}), I_{2}(0,0, \tilde{r}), \tilde{I}_{2}(0,0,0 ; \tilde{r}), \tilde{I}_{2}(0, i, i ; \tilde{r})$ and $\tilde{I}_{2}(k, 2 i, 0 ; \tilde{r})$. In that case, setting $k=0$ and $\epsilon=0$ in the $O\left(\epsilon^{0}\right)$ terms from $I_{2}(k, 2 i ; \tilde{r})$ we find:

$$
I_{2}(0,2 i, \tilde{r})=\mu^{-\epsilon} \frac{1}{\epsilon}\left(1-\frac{\epsilon}{2}-\frac{\epsilon}{2} \int_{0}^{1} \ln \left[4 x(1-x) \tilde{r}^{2} i^{2}+1\right] d x+\frac{\epsilon}{2} \tilde{F}_{0}(0, i, \tilde{r})\right) .
$$

It is also simple to demonstrate that $\tilde{I}_{2}(0,0,0 ; \tilde{r})=\frac{\tilde{r} \mu^{-\epsilon}}{2}, \tilde{I}_{2}(k, 2 i, 0 ; \tilde{r})=\frac{\tilde{r} \mu^{-\epsilon}}{1+\sqrt{1+4 \tilde{r}^{2} i^{2}}}$ and $\tilde{I}_{2}(k, i, i ; \tilde{r})=\frac{\tilde{r} \mu^{-\epsilon}}{2 \sqrt{1+\tilde{r}^{2} i^{2}}}$. Replacing these results in the expression of the one-loop four-point diagram for the particular choices of external momenta and quasi-momenta, we can write

$$
\begin{aligned}
& { }_{i}^{i} 久_{i}^{i}=3 \tilde{\sigma} \frac{(N+8)}{9 \epsilon} \mu^{-\epsilon}\left[1-\frac{\epsilon}{2}+\epsilon \zeta^{(\tau)}(i ; \tilde{r})\right], \\
& \zeta^{(\tau)}(i ; \tilde{r})=\frac{1}{6} \tilde{F}_{0}(0,2 i, \tilde{r})+\frac{1}{3} \tilde{F}_{0}(0,0, \tilde{r})+\frac{\tilde{r} \tau}{3}+\frac{2 \tilde{r} \tau}{3\left[1+\sqrt{1+4 \tilde{r}^{2} i^{2}}\right]}+\frac{2 \tilde{r}}{3 \sqrt{1+\tilde{r}^{2} i^{2}}} \\
& -\frac{1}{3}\left[\frac{\sqrt{1+\tilde{r}^{2} i^{2}}}{\tilde{r} i} \operatorname{arcsinh}(\tilde{r} i)-1\right] \text {. }
\end{aligned}
$$

In the above equation $\tilde{r} \propto\left(\frac{L}{\xi}\right)^{-1}$ where $\xi\left(\sim \mu^{-1}\right)$ is the bulk (infinite system) correlation length. We will analyze this correction term in order to study its asymptotical behavior for large as well as small values of $L$.

Let us start by the first scaling regime, namely $\frac{L}{\xi} \rightarrow \infty(\tilde{r} \rightarrow 0)$ limit. It is easy to demonstrate that $i$ ) the linear terms in $\tilde{r}$ go to zero, ii) $\tilde{F}_{0}(0,0, \tilde{r} \rightarrow 0) \rightarrow 0$ [22] and iii) the last term also vanishes. It is not difficult to demonstrate that $\tilde{F}_{0}(0,2 i, \tilde{r} \rightarrow 0)$ tends to zero as follows. From Eq. (76c), $\lim _{\tilde{r} \rightarrow 0} h(0,2 i, \tilde{r}) \rightarrow \tilde{r}^{-1}$, and we can solve the integral by writing explicitly the integrand in terms of a summation involving Bessel functions. The coefficient of each term in the summation is equal to $\sin (4 \pi m i)$ which is zero for integer $i$, therefore proving the assertion. Then, the finite-size correction reduces to the bulk result $\left(\zeta^{(\tau)}(i ; \tilde{r} \rightarrow 0)=0\right)$ whenever $\frac{L}{\xi} \rightarrow \infty$.

Next we consider the limit $\frac{L}{\xi} \rightarrow 0(\tilde{r} \rightarrow \infty)$. The several terms inside the correction function are going to be examined separately. Note that $\lim _{\tilde{r} \rightarrow \infty} \frac{2 \tilde{r} \tau}{3\left[1+\sqrt{1+4 \tilde{r}^{2} i^{2}}\right]} \rightarrow \frac{\tau}{3 i}, \lim _{\tilde{r} \rightarrow \infty} \frac{2 \tilde{r}}{3 \sqrt{1+\tilde{r}^{2} i^{2}}} \rightarrow \frac{2}{3 i}$, 
which are convergent and $\tilde{r}$-independent. In addition, $\lim _{\tilde{r} \rightarrow \infty}-\frac{1}{3}\left[\frac{\sqrt{1+\tilde{r}^{2} i^{2}}}{\tilde{r} i} \operatorname{arcsinh}(\tilde{r} i)-1\right] \rightarrow$ $-\frac{1}{3}[\ln (2 \tilde{r} i)-1]$, and we have a logarithmic divergence in the desired limit.

We now focus on the contributions from $\tilde{F}_{0}(0,2 i, \tilde{r})$ and $\tilde{F}_{0}(0,0, \tilde{r})$ in this limit. The function

$$
f_{\frac{1}{2}}\left(2 i x, h(k=0,2 i, \tilde{r})=4 \sum_{m=1}^{\infty} \cos (4 \pi m i x) K_{0}(2 \pi m h(k=0,2 i, \tilde{r}),\right.
$$

can be better understood through the identity [31]

$$
\begin{aligned}
& \sum_{n=1}^{\infty} K_{0}(n \hat{x}) \cos (n \hat{x} t)=\frac{1}{2}\left[\gamma+\ln \left(\frac{\hat{x}}{4 \pi}\right)\right]+\frac{\pi}{2 \hat{x} \sqrt{1+t^{2}}} \\
& +\frac{\pi}{2} \sum_{n=1}^{\infty}\left[\frac{1}{\sqrt{\hat{x}^{2}+(2 n \pi+t \hat{x})^{2}}}-\frac{1}{2 n \pi}\right]+\frac{\pi}{2} \sum_{n=1}^{\infty}\left[\frac{1}{\sqrt{\hat{x}^{2}+(2 n \pi-t \hat{x})^{2}}}-\frac{1}{2 n \pi}\right]
\end{aligned}
$$

where $\gamma=0.57721566 \ldots$ is the Euler-Mascheroni constant. It is valid for positive values of the variable $\hat{x}$. Performing the the identifications $\hat{x}=2 \pi \tilde{r}^{-1} \sqrt{1+4 \tilde{r}^{2} i^{2} x(1-x)}, t=$ $\frac{2 \tilde{r} i x}{\sqrt{1+4 \tilde{r}^{2} i^{2} x(1-x)}}$ we get to

$$
\begin{aligned}
& f_{\frac{1}{2}}\left(2 i x, h(k=0,2 i, \tilde{r})=2 \gamma+2 \ln \left[\frac{\sqrt{1+4 \tilde{r}^{2} i^{2} x(1-x)}}{2 \tilde{r}}\right]+\frac{\tilde{r}}{\sqrt{1+4 \tilde{r}^{2} i^{2} x}}\right. \\
& +\sum_{n=1}^{\infty}\left[\frac{1}{\sqrt{\tilde{r}^{-2}+4 i(i-n) x+n^{2}}}-\frac{1}{n}\right]+\sum_{n=1}^{\infty}\left[\frac{1}{\sqrt{\tilde{r}^{-2}+4 i(i+n) x+n^{2}}}-\frac{1}{n}\right] .
\end{aligned}
$$

In particular, for $i=0$

$$
f_{\frac{1}{2}}\left(0, h(k=0,0, \tilde{r})=2 \gamma-2 \ln (2 \tilde{r})+\tilde{r}+2 \sum_{n=1}^{\infty}\left[\frac{1}{\sqrt{\tilde{r}^{-2}+n^{2}}}-\frac{1}{n}\right] .\right.
$$

This coincides exactly with $\tilde{F}_{0}(0,0, \tilde{r})$. Taking the limit (setting $\tilde{r}=\infty$ into the last term) we find $\lim _{\tilde{r} \rightarrow \infty} \tilde{F}_{0}(0,0, \tilde{r}) \rightarrow \tilde{r}-2 \ln (2 \tilde{r})$. Finally, we have to analyze $\tilde{F}_{0}(0,2 i, \tilde{r})$ which is given by

$$
\begin{aligned}
& \tilde{F}_{0}(k=0,2 i, \tilde{r})=2 \gamma+2 \int_{0}^{1} d x \ln \left[\frac{\sqrt{1+4 \tilde{r}^{2} i^{2} x(1-x)}}{2 \tilde{r}}\right]+\int_{0}^{1} d x \frac{\tilde{r}}{\sqrt{1+4 \tilde{r}^{2} i^{2} x}}+\sum_{n=1}^{\infty} \int_{0}^{1} d x \\
& \times\left[\frac{1}{\sqrt{\tilde{r}^{-2}+4 i(i-n) x+n^{2}}}-\frac{1}{n}\right]+\sum_{n=1}^{\infty} \int_{0}^{1} d x\left[\frac{1}{\sqrt{\tilde{r}^{-2}+4 i(i+n) x+n^{2}}}-\frac{1}{n}\right] .
\end{aligned}
$$

One can show that $\lim _{\tilde{r} \rightarrow \infty} \int_{0}^{1} d x \ln \left[\frac{\sqrt{1+4 \tilde{r}^{2} i^{2} x(1-x)}}{2 \tilde{r}}\right] \rightarrow \ln i-1$, which is finite in this limit. The third term can also be shown to be finite, namely, $\left.\lim _{\tilde{r} \rightarrow \infty} \int_{0}^{1} d x \frac{\tilde{r}}{\sqrt{1+4 \tilde{r}^{2} i^{2} x}} \rightarrow\left(\frac{1}{i}\right)\right)$. 
The summations conceal an underlying subtlety for $i \neq 0$. Looking at Eq. (83) there was no problem whatsoever in replacing directly $\tilde{r}=\infty$ when $i=0$ at the summations. That was the way we renormalized $P B C$ and $A B C$ with $i=0$. Indeed, setting directly $(r=2 \tilde{r}$ in those boundary conditions) $\tilde{r}^{-2}=0$, these terms contribute zero to $P B C$ (just as here) and $(2 \ln 2-1)$ for $A B C$. In other words, they contribute a finite constant $\tilde{r}$-independent, which do not have a pathological behavior in the limit $\tilde{r} \rightarrow \infty$. Looking at $P B C$, if we do not choose to renormalize the theory at $i=0$, but choose instead all external quasi-momenta equal to $i>0$, then the form of these summations is identical to those which appear in $D B C$ and $N B C$ here. In $A B C$ the occurrence of this feature is a little bit different since one has to add the finite term above mentioned.

Now consider $i \neq 0$ and see what happens when we set $\tilde{r}^{-2}=0$ in the summations. Using the power series expansion from $(1+y)^{-\frac{1}{2}}$, keeping all terms in $y$ and performing the integrals over the Feynman parameter $x$, we obtain

$$
\begin{gathered}
\lim _{\tilde{r} \rightarrow \infty}\left(\sum_{n=1}^{\infty} \int_{0}^{1} d x\left[\frac{1}{\sqrt{\tilde{r}^{-2}+4 i(i-n) x+n^{2}}}-\frac{1}{n}\right]+\sum_{n=1}^{\infty} \int_{0}^{1} d x\left[\frac{1}{\sqrt{\tilde{r}^{-2}+4 i(i+n) x+n^{2}}}-\frac{1}{n}\right]\right)= \\
2 \sum_{n=1}^{\infty} i^{2 n} \zeta(2 n+1),
\end{gathered}
$$

where $\zeta(2 n+1)=\sum_{p=1}^{\infty} \frac{1}{p^{2 n+1}}$.

It is clear that the series diverges for $i \geq 1$. This divergent number is harmless in the limit $\tilde{r} \rightarrow \infty$, since $i$ ) it does not depend on $\tilde{r}$, ii) it can be eliminated by suitable choices of external quasi-momenta in $P B C, N B C$ and $A B C(e . g ., i=0)$. As far as the series does not depend explicitly on $\tilde{r}$, it can be safely considered regular in this limit in comparison with poles in $\epsilon$. In order to eliminate the divergent term for $P B C, A B C, D B C$ and $N B C$ when we choose $i \neq 0$, which is clearly an artifact of the method, we can define a "normal ordered" (regularized) correction : $\zeta^{(\tau)}(i, \tilde{r}):=\zeta^{(\tau)}(i, \tilde{r})-\frac{1}{3} \sum_{n=1}^{\infty} i^{2 n} \zeta(2 n+1)$ that is still divergent in the limit $L \rightarrow 0$ but without the nonphysical divergence associated to this summation. This normal ordering operation ammounts to say that if one replaces directly $\tilde{r}=\infty$ in the infinite sumations Eq. (81) (or alternatively into Eqs. (82), (84)) whenever $i \neq 0$ in analogy with which is done in the case $i=0$, one shoud be careful to subtract this term aformentioned. That is the actual quantity that should be used in the limit of small values of $L$.

Putting together all the terms examined in this limit, we find that the regularized finite- 
size correction function reduces to

$$
\lim _{\tilde{r} \rightarrow \infty}: \zeta^{(\tau)}(i, \tilde{r}): \rightarrow \frac{1}{6 i}(2 \tau+5)-\ln (2 \tilde{r})+\frac{\tilde{r}(1+\tau)}{3} .
$$

The finite-size correction is clearly boundary condition dependent. From this expression we can obtain two distinct behaviors in this limit whenever the system is not located at the bulk critical temperature $(t \neq 0)$. For $D B C(\tau=-1)$, the divergence is purely logarithimic like in $A B C$, whereas the dominant term for $N B C(\tau=1)$ diverges linearly with $\tilde{r}$ just like in $P B C$. When these terms become comparable to the dimensional pole in $\epsilon$ through decreasing values of $L$ the dimensional crossover takes place.

Let us turn now our attention to the two- and three-loop diagrams. They are required to getting the critical exponents $\eta$ and $\nu$ perturbatively in the safe scaling region discussed above where $L$ is not so small and $\epsilon$-expansion results are valid. We will just write down the solution of the integrals. The interested reader should consult the details in Appendix A.

Consider the two-loop diagrams of the four-point vertex function. They consist of "diagonal terms" which are responsible for the leading singularities in $\epsilon$ as well as "nondiagonal terms". The double bubble can be easily obtained from the one-loop diagram just discussed by noting that the last piece comprising the products of two $\tilde{I}_{2}$ 's and including an infinite summation are nonsingular in $\epsilon$ and can be neglected away from the crossover region. We then find at zero external momenta and special external quasi-momenta $i$ the following result

$$
{ }_{i}^{i} \bigcirc \mathcal{X}_{i}^{i}=3 \tilde{\sigma} \frac{\left(N^{2}+6 N+20\right)}{27} \mu^{-2 \epsilon}\left\{\frac{1}{\epsilon^{2}}\left(1-\epsilon+2 \epsilon \zeta^{(\tau)}(i, \tilde{r})\right)\right\} \text {. }
$$

A similar observation permits our computation of the nontrivial two-loop diagram since the integrals $\hat{I}_{4}$ do not contribute to the singular terms of this graph. At zero external momenta and nonvanishing external quasi-momenta set to the value $i$ in all external legs, it turns out to be given by:

$$
\overbrace{i}^{i}=3 \tilde{\sigma} \frac{(5 N+22)}{27} \mu^{-2 \epsilon}\left\{\frac{1}{2 \epsilon^{2}}\left(1-\frac{\epsilon}{2}+2 \epsilon \zeta^{(\tau)}(i, \tilde{r})\right)\right\}
$$

In normalization conditions, we shall need the derivative of the two-point vertex part diagrams computed at zero external momenta in this massive framework. The "sunsetting" two-loop diagram involves the integral $\frac{I_{3}(k=0, i, \tilde{\sigma}, \mu)}{S_{d}^{2}} \equiv I_{3}(k=0, i, \tilde{r})$ and we are interested in the object $\left.I_{3}^{\prime}(i, \tilde{r}) \equiv \frac{\partial I_{3}(k, i, \tilde{r})}{\partial k^{2}}\right|_{k=0}$ together with the other tilded integrals . The three-loop graph has a similar structure in terms of the integrals $\frac{I_{5}(k=0, i, \tilde{\sigma}, \mu)}{S_{d}^{3}} \equiv I_{5}(k=0, i, \tilde{r})$. The 
utilization of the combinations previously prescribed as well as the result of the integrals in the Appendix A imply that those diagrams can be cast in the form

$$
\begin{aligned}
& \frac{\partial}{\partial k^{2}}\left(\bigcirc_{i}\right)_{k^{2}=0}=-\frac{\mu^{-2 \epsilon}(N+2)}{24 \epsilon}\left[1-\frac{\epsilon}{4}+\epsilon \tilde{W}^{(\tau)}(i, \tilde{r})\right], \\
& \frac{\partial}{\partial k^{2}}\left({ }_{i}\right)_{k^{2}=0}=-\frac{\mu^{-3 \epsilon}(N+2)(N+8)}{162 \epsilon^{2}}\left[1-\frac{\epsilon}{4}+\frac{3 \epsilon}{2} \tilde{W}^{(\tau)}(i ; \tilde{r})\right] .
\end{aligned}
$$

Some useful definitions similar to those occurring in $P B C$ and $A B C$ boundary conditions are:

$$
\begin{aligned}
& \tilde{F}_{\alpha, \beta}^{(\tau)}(k, i ; \tilde{r}) \equiv \frac{1}{S_{d}} \tilde{r} \sum_{j=-\infty}^{\infty} \int d^{d-1} q \frac{\tilde{F}_{\alpha}^{(\tau)}(q+k, j+i ; \tilde{r})}{\left[(q)^{2}+\tilde{r}^{2} j^{2}+1\right]^{\beta}} \\
& \left.\tilde{F}_{\alpha}^{\prime(\tau)}(i ; \tilde{r}) \equiv \frac{\partial \tilde{F}_{\alpha, 1}^{(\tau)}(k, i ; \tilde{r})}{\partial k^{2}}\right|_{k=0}, \\
& \tilde{\mathcal{F}}_{\alpha, \beta}^{(\tau)}(k, j, i ; \tilde{r}) \equiv \frac{1}{S_{d}} \tilde{r} \int d^{d-1} q \frac{\tilde{F}_{\alpha}^{(\tau)}(q+k, i ; \tilde{r})}{\left[(q)^{2}+\tilde{r}^{2} j^{2}+1\right]^{\beta}} \\
& \left.\tilde{\mathcal{F}}_{\alpha}^{\prime(\tau)}(i, j ; \tilde{r}) \equiv \frac{\partial \tilde{\mathcal{F}}_{\alpha, 1}^{(\tau)}(k, i, j ; \tilde{r})}{\partial k^{2}}\right|_{k=0} .
\end{aligned}
$$

When expressed in terms of these definitions, the above finite size correction to the higher loop two-point graphs represented by the amount $\tilde{W}^{(\tau)}(i, \tilde{r})$ can be written as

$$
\begin{aligned}
& \tilde{W}^{(\tau)}(i ; \tilde{r})=-\frac{1}{2}-2 \int_{0}^{1} \int_{0}^{1} d x d y(1-y) \ln \left[y(1-y) i^{2}+(1-y) \tilde{r}^{-2}+\frac{y \tilde{r}^{-2}}{x(1-x)}\right] \\
& +2 \int_{0}^{1} \int_{0}^{1} d x d y(1-y) f_{\frac{1}{2}}\left(i y, \sqrt{y(1-y) i^{2}+(1-y) \tilde{r}^{-2}+\frac{y \tilde{r}^{-2}}{x(1-x)}}\right)-4 \tilde{F}_{0}^{\prime}(i ; \tilde{r}) \\
& +6 \tilde{r}\left[\int_{0}^{1} \int_{0}^{1} d x d y(1-y)\left(\tilde{r}^{2} i^{2}(1-y)+y\left(\frac{1}{x(1-x)}-1\right)+1\right)^{-\frac{1}{2}}\right. \\
& \left.+\tau \int_{0}^{1} \int_{0}^{1} d x d y(1-y)\left(\tilde{r}^{2} i^{2} y+y\left(\frac{1}{x(1-x)}-1\right)+1\right)^{-\frac{1}{2}}\right] \\
& -12\left[\mathcal{F}_{0}^{\prime(\tau)}(i, 0 ; \tilde{r})+\tau \mathcal{F}_{0}^{\prime(\tau)}(0, i ; \tilde{r})\right]
\end{aligned}
$$

Finally let us present the solution of the vertex $\Gamma^{(2,1)}$ graphs at a suitable symmetry point (zero external momenta). These diagrams can be written in terms of those integrals of the four-point function. For instance, the one-loop diagram, according to the rule outlined in the diagrammatic Eq. (38) can be written as

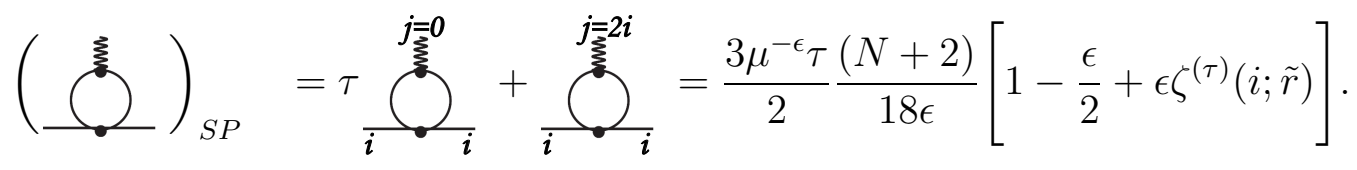


We are also interested in the solution for the two-loop diagrams. Using the same rule, we first obtain for the trivial two-loop diagram

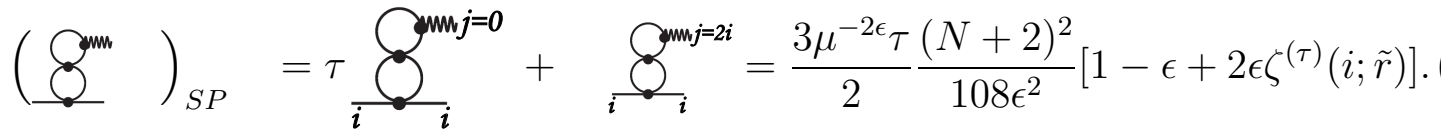

The nontrivial two-loop, on the other hand is given by:

$$
(\bigcup_{S P}=\tau \overbrace{i}^{j=0}+\bigodot_{i}^{j=2 i}=\frac{3 \mu^{-2 \epsilon} \tau}{2} \frac{(N+2)}{72 \epsilon^{2}}\left[1-\frac{\epsilon}{2}+2 \epsilon \zeta^{(\tau)}(i ; \tilde{r})\right] \text {. }
$$

A glance in the results obtained from the graphs analyzed so far demonstrates the nontrivial character of the finite-size correction terms for $D B C$ and $N B C$ in comparison to the $P B C$ and $A B C$ counterparts. The nondiagonal terms produce singular contributions at two-loop order in all primitively divergent vertex functions (and three-loop order in the two-point function) which modify the correction terms from the periodic and antiperiodic cases.

With these data we can proceed to compute the critical exponents at least up to two-loop level. We carry out this task in the following section.

\section{IV. $D B C$ AND $N B C$ CRITICAL EXPONENTS IN THE MASSIVE FINITE-SIZE REGIME}

In order to compute the fixed point and finally the critical exponents in a parallel plate geometries with one direction of finite extent in the apropriate regime, we write the dimensionless bare coupling constant in terms of the renormalized one as $u_{0}^{(\tau)}=u\left(1+a_{1}^{(\tau)} u+a_{2}^{(\tau)} u^{2}\right)$. Although we are going to show that the fixed point is boundary condition dependent, we shall omit henceforth the explicit dependence on $\tau$ in both bare and renormalized dimensionless coupling constants. The renormalization functions are written as $Z_{\phi}^{(\tau)}=1+b_{2}^{(\tau)} u^{2}+b_{3}^{(\tau)} u^{3}$ and $\bar{Z}_{\phi^{2}}^{(\tau)}=1+c_{1}^{(\tau)} u+c_{2}^{(\tau)} u^{2}$.

The bare vertex parts which can be renormalized multiplicatively at zero external momenta and nonvanishing external quasi-momenta $i$ can be written in the form

$$
\begin{aligned}
& \left.\frac{\partial \tilde{\Gamma}^{(2)}}{\partial k^{2}}\right|_{k^{2}=0}=1-\tilde{B}_{2}^{(\tau)} u_{0}^{2}+\tilde{B}_{3}^{(\tau)} u_{0}^{3} \\
& \left.\Gamma^{(4)}\right|_{S P}=3 \tilde{\sigma} u_{0} \mu^{\epsilon}\left[1-\tilde{A}_{1}^{(\tau)} u_{0}+\left(\tilde{A}_{2}^{(1 \tau)}+\tilde{A}_{2}^{(2 \tau)}\right) u_{0}^{2}\right] \\
& \left.\Gamma^{(2,1)}\right|_{\overline{S P}}=\frac{3 \tau}{2}-\tilde{C}_{1}^{(\tau)} u_{0}+\left(\tilde{C}_{2}^{(1 \tau)}+\tilde{C}_{2}^{(2 \tau)}\right) u_{0}^{2} .
\end{aligned}
$$


In the previous equations, the following identifications can be made:

$$
\begin{aligned}
& \tilde{B}_{2}^{(\tau)}=\frac{\mu^{2 \epsilon}}{6} \frac{\partial}{\partial k^{2}}(\overbrace{i})_{k^{2}=0}=-\frac{(N+2)}{144 \epsilon}\left[1-\frac{\epsilon}{4}+\epsilon \tilde{W}^{(\tau)}(i, \tilde{r})\right] \\
& \tilde{B}_{3}^{(\tau)}=\frac{\mu^{3 \epsilon}}{4} \frac{\partial}{\partial k^{2}}\left({ }_{i}\right)_{k^{2}=0} ;=-\frac{(N+2)(N+8)}{648 \epsilon^{2}}\left[1-\frac{\epsilon}{4}+\frac{3 \epsilon}{2} \tilde{W}^{(\tau)}(i, \tilde{r})\right] \text {, } \\
& \tilde{A}_{1}^{(\tau)}=\frac{\mu^{\epsilon}}{2 \tilde{\sigma}}{ }_{i} \mathcal{X}_{i}^{i}=\frac{(N+8)}{6 \epsilon}\left[1-\frac{\epsilon}{2}+\epsilon \zeta^{(\tau)}(i ; \tilde{r})\right] \\
& \tilde{A}_{2}^{(1 \tau)}=\frac{\mu^{2 \epsilon}}{4 \tilde{\sigma}}{ }_{i} \gamma \bigcirc \mathcal{K}_{i}^{i} \frac{\left(N^{2}+6 N+20\right)}{36}\left\{\frac{1}{\epsilon^{2}}\left(1-\epsilon+2 \epsilon \zeta^{(\tau)}(i, \tilde{r})\right)\right\} \text {, } \\
& \left.\tilde{A}_{2}^{(2 \tau)}\right)=\frac{\mu^{2 \epsilon}}{\tilde{\sigma}} \bigodot_{i}^{i}=\frac{(5 N+22)}{18 \epsilon^{2}}\left(1-\frac{\epsilon}{2}+2 \epsilon \zeta^{(\tau)}(i, \tilde{r})\right) \text {, } \\
& \tilde{C}_{1}^{(\tau)}=3 \mu^{\epsilon}(\underbrace{\stackrel{\xi}{3}}_{S P})_{S}=\frac{3 \tau}{2} \frac{(N+2)}{6 \epsilon}\left[1-\frac{\epsilon}{2}+\epsilon \zeta^{(\tau)}(i ; \tilde{r})\right] \text {, } \\
& \tilde{C}_{2}^{(1 \tau)}=3 \mu^{2 \epsilon}(\underbrace{\text { mm }})_{S P}=\frac{3 \tau}{2} \frac{(N+2)^{2}}{36 \epsilon^{2}}\left[1-\epsilon+2 \epsilon \zeta^{(\tau)}(i ; \tilde{r})\right] . \\
& \tilde{C}_{2}^{(2 \tau)}=6 \mu^{2 \epsilon}()_{S P}=\frac{3 \tau}{2} \frac{(N+2)}{12 \epsilon^{2}}\left[1-\frac{\epsilon}{2}+2 \epsilon \zeta^{(\tau)}(i ; \tilde{r})\right] \text {. }
\end{aligned}
$$

Next, we apply the normalization conditions Eqs. (170). Using the definition of the renormalized vertex parts discussed before, we can determine the normalization functions by imposing finiteness of the renormalized quantities. We start by replacing the dimensionless bare coupling constant in the diagrammatic expansion of the two- and four-point function. Use the definition from $Z_{\phi}^{(\tau)}$ into Eq. (70b) in order to determine $b_{2}^{(\tau)}$. Replace the dimensionless bare coupling constant by the renormalized one at $O\left(u_{0}^{2}\right)$ since the correction will be $O\left(u^{3}\right)$ and can be neglected. We then find

$$
b_{2}^{(\tau)}=-\frac{(N+2)}{144 \epsilon}\left[1-\frac{\epsilon}{4}+\epsilon \tilde{W}^{(\tau)}(i, \tilde{r})\right] .
$$

Using the above expression in the definition of the renormalized four-point function is sufficient to obtain

$$
\begin{aligned}
& a_{1}^{(\tau)}=\frac{(N+8)}{6 \epsilon}\left[1-\frac{\epsilon}{2}+\epsilon \zeta^{(\tau)}(i ; \tilde{r})\right] \\
& a_{2}^{(\tau)}=\left[\frac{(N+8)}{6 \epsilon}\right]^{2}\left[1+2 \epsilon \zeta^{(\tau)}(i ; \tilde{r})\right]-\frac{\left(2 N^{2}+41 N+170\right)}{72 \epsilon} .
\end{aligned}
$$

Using these data, we can compute $b_{3}^{(\tau)}$ which can be shown to be given by

$$
b_{3}^{(\tau)}=-\frac{(N+2)(N+8)}{1296 \epsilon^{2}}\left[1-\frac{7 \epsilon}{4}+3 \epsilon \zeta^{(\tau)}(i ; \tilde{r})\right]
$$


The renormalization function which defines the renormalized composite field can be determined in a very similar manner using the above expressions for $a_{1}^{(\tau)}, a_{2}^{(\tau)}$ and the diagrammatic expansion for that vertex part. We then find

$$
\begin{aligned}
& c_{1}^{(\tau)}=\frac{(N+2)}{6 \epsilon}\left[1-\frac{\epsilon}{2}+\epsilon \zeta^{(\tau)}(i ; \tilde{r})\right], \\
& c_{2}^{(\tau)}=\frac{(N+2)(N+5)}{36 \epsilon^{2}}-\frac{(N+2)(2 N+13)}{72 \epsilon}+\frac{\left(N^{2}+7 N+10\right)}{18 \epsilon} \zeta^{(\tau)}(i ; \tilde{r}) .
\end{aligned}
$$

The Wilson functions defined in Eqs. (73) can be written in terms of these coefficients as

$$
\begin{aligned}
& \beta^{(\tau)}(u)=-\epsilon u\left[1-a_{1}^{(\tau)} u+2\left(\left(a_{1}^{(\tau)}\right)^{2}-a_{2}^{(\tau)}\right) u^{2}\right], \\
& \gamma_{\phi}^{(\tau)}(u)=-\epsilon u\left[2 b_{2}^{(\tau)}+\left(3 b_{3}^{(\tau)}-2 b_{2}^{(\tau)} a_{1}^{(\tau)}\right) u^{2}\right], \\
& \bar{\gamma}_{\phi^{2}}^{(\tau)}(u)=\epsilon u\left[c_{1}^{(\tau)}+\left(2 c_{2}^{(\tau)}-\left(c_{1}^{(\tau)}\right)^{2}-a_{1}^{(\tau)} c_{1}^{(\tau)}\right) u\right] .
\end{aligned}
$$

In order to evaluate the critical exponents, we need the fixed point of the dimensionless coupling constant, which is determined by the condition $\beta^{(\tau)}\left(u_{\infty}\right)=0$. It is a simple task to demonstrate that it is given by the following expression:

$$
u_{\infty}=\left[\frac{6 \epsilon}{N+8}\right]\left\{1+\left[\frac{(9 N+42)}{(N+8)^{2}}+\frac{1}{2}-\zeta^{(\tau)}(i ; \tilde{r})\right] \epsilon\right\} .
$$

The exponent $\eta$ is identified with $\gamma_{\phi}^{(\tau)}\left(u_{\infty}\right)$ which implies the following three-loop result

$$
\eta \equiv \gamma_{\phi}^{(\tau)}\left(u_{\infty}\right)=\frac{(N+2)}{2(N+8)^{2}} \epsilon^{2}\left\{1+\epsilon\left[\frac{6(3 N+14)}{(N+8)^{2}}-\frac{1}{4}\right]\right\}
$$

The other Wilson function at the fixed point is given by $\bar{\gamma}_{\phi^{2}}^{(\tau)}\left(u_{\infty}\right)=\frac{(N+2)}{(N+8)} \epsilon\left[1+\frac{(6 N+18)}{(N+8)^{2}} \epsilon\right]$. The exponent $\nu$ is related to the exponent $\eta$ and the last expression through $\nu^{-1}=2-\bar{\gamma}_{\phi^{2}}^{(\tau)}\left(u_{\infty}\right)-\eta$, which yields

$$
\nu=\frac{1}{2}+\frac{(N+2)}{4(N+8)} \epsilon+\frac{(N+2)\left(N^{2}+23 N+60\right)}{8(N+8)^{3}} \epsilon^{2} .
$$

These universal results are independent of the boundary conditions and reproduce the bulk critical exponents. Despite the fixed point and the Wilson functions carry residual nonuniversal information from the normalization conditions employed encoded in the nontrivial correction function $\zeta^{(\tau)}(i ; \tilde{r})$, the cancellations take place exactly to eliminate all the finite-size information resulting in the bulk critical exponents. A similar feature was shown to be valid for $P B C$ and $A B C$. In the present case, even though the boundary conditions have not the same smooth character from $P B C$ and $A B C$, we have just proven that the 
previous conjecture made by Nemirovsky and Freed that the critical exponents are the same from the bulk theory also for $D B C$ and $N B C$.

In order to complete the analysis of the critical behavior from finite-size systems confined between parallel plates, let us discuss the situation from the viewpoint of masless fields, generalizing the picture just developed involving only massive fields. We shall use other set of normalization conditions and will show that the critical exponents agree with those obtained in the present section.

\section{MASSLESS FIELDS FORMULATION: DBC AND NBC IN THE EXPONEN- TIAL REPRESENTATION}

We have determined in previous sections the exact form of the several diagrams in perturbation theory. Now we perform the substitution of the massive integrals by their zero mass analogues. Then, it is possible to define a simple set of normalization conditions which allow us to consider the same type of diagrams already considered in the massive framework.

Let us get started by describing the normalization conditions. The restriction to the minimal number of diagrams is analogous to what was discussed in the massive theory. We define the quantity $\tilde{\Gamma}^{(2)}(k, j, \mu=0, \tilde{\sigma}) \equiv \Gamma^{(2)}(k, j, \mu=0, \tilde{\sigma})-\tilde{\sigma}^{2} j^{2}$. This means that the term $\tilde{\sigma}^{2} j^{2}$ does not need to be renormalized by the normalization functions, just like we did for the massive fields. (We careless kept the original vertex $\Gamma^{(2)}(k, j=0, \mu=0, \sigma)$ in our treatment for $A B C$ and $P B C$ such that the similar term $\sigma^{2} \tau^{2}$ appeared therein. It has no effect for $P B C$, but produces a small deviation in the normalization as discussed in Ref. [22] for $A B C$. The correct form to get rid of this inconvenience is to define the object $\tilde{\Gamma}^{(2)}(k, j=0, \mu=0, \sigma) \equiv \Gamma^{(2)}(k, j=0, \mu=0, \sigma)-\sigma^{2} \tau^{2}$ for $A B C$ as we did above for $D B C$ and $N B C$. This maneuver do not alter the normalization constants obtained in

Ref. [22], since the argument there implicitly took into account this feature). We choose a symmetry point with the following properties: i) since the theory now possesses infrared divergences, we have to renormalize the primitively divergent vertices at nonzero external momenta; ii) we also choose nonvanishing external quasi-momenta owing to the boundary conditions. If $k_{i}$ are the external momenta associated to the infinite $(d-1)$-dimensional subspace and $j$ is the moding attached to the external quasi-momentum characterizing the distance between the plates, the theory is renormalized at fixed external momentum scale 
$\kappa$ and arbitrary nonvanishing moding $i$. The symmetric point is defined on the infinite subspace with the condition $k_{i} \cdot k_{j}=\frac{\kappa^{2}}{4}\left(4 \delta_{i j}-1\right)$, whereas all the external quasi-momenta mode of any primitively divergent one-particle irreducible $(1 P I)$ vertex part is chosen in the value $i$ (except for $\Gamma^{(2,1)}$; see below).

The multiplicative renormalization can be successfully implemented through the following normalization conditions on the primitively divergent vertex functions:

$$
\begin{aligned}
& \tilde{\Gamma}_{R}^{(2)}(k=0, i, g, 0)=0, \\
& \left.\frac{\partial \tilde{\Gamma}_{R}^{(2)}(k=\kappa, j, g, 0)}{\partial k^{2}}\right|_{k^{2}=\kappa^{2}}=1, \\
& \left.\Gamma_{R}^{(4)}\left(k_{l}, i_{l}=i, g, 0\right)\right|_{S P}=3 \tilde{\sigma} g, \\
& \left.\Gamma_{R}^{(2,1)}\left(k_{1}, i_{1}=i, k_{2}, i_{2}=i, Q, j, g, 0\right)\right|_{S P}=3 \frac{\tau}{2} .
\end{aligned}
$$

Note that the symmetry point is such that the insertion momentum is related to the other momenta in last equation through $Q^{2}=\left(k_{1}+k_{2}\right)^{2}$. Second, recall from our discussion from the massive fields that the same rule applies for the vertex $\left.\Gamma_{R}^{(2,1)}\left(k_{1}, i_{1}, k_{2}, i_{2}, Q, j, g, 0\right)\right|_{\overline{S P}}$ : the tree-level vertex diagram corresponds to the combination

$$
\sum_{1--2}^{3}=\left.\sum_{1--2}^{3}\right|_{\left(j= \pm\left(j_{1}+j_{2}\right)\right.}+\left.\tau \sum_{1--2}^{3}\right|_{(j=0)},
$$

where the signal of $j$ is fixed. We attach to the external legs quasi-momentum $i$, such that the insertion quasi-momentum has two contributions: either $j=2 i$ or $j=0$.

In order to get rid of the mass insertions, we can follow two different trends. The first one resembles the massive theory and can be formulated as follows. Start with a tree-level bare mass $\mu_{0}$. Impose the condition $\tilde{\Gamma}^{(2)}\left(k=0, \mu_{0}, j, \lambda_{0}\right)=\tilde{\mu}_{0}^{2}$. Invert this equation to obtain $\mu_{0}\left(\tilde{\mu}_{0}\right)$ just as we done in the massive case. This $\mu_{0}$ could be interpreted as the shift in the bulk critical temperature. Replace this back into the diagrammatic expression of the vertex part $\tilde{\Gamma}^{(2)}\left(k=0, \mu_{0}, j, \lambda\right)=\tilde{\mu}_{0}^{2}$. This eliminates all tadpole diagrams. Next, express $\mu_{0}\left(\tilde{\mu}_{0}\right)$ up to $O\left(\lambda_{0}\right)$ and Taylor expand the remaining vertex part around $k^{2}=\kappa^{2}$. The net effect is to eliminate all mass insertions in all primitively divergent vertex parts. Finally set $\tilde{\mu}_{0}=0$.

Although this argument makes perfect sense from the point of view of statistical mechanics where the mass is identified with the reduced temperature (distance to the critical temperature), the reader working on quantum field theory might feel uneasy by starting with a nonvanishing bare mass, defining a three-loop bare mass and set it to zero afterwards, since it resembles a dynamical mass generation induced perturbatively. It is then 
worthwhile to develop the most traditional argument in which the masss is zero in all orders in perturbation theory as we will describe now.

Let us start directly with $\mu_{0}=0$, which implies $\tilde{\Gamma}^{(2)}\left(k=0, \mu_{0}=0, j, \lambda_{0}\right)=0$. Consequently, one finds the diagrammatic expression up to three-loop order:

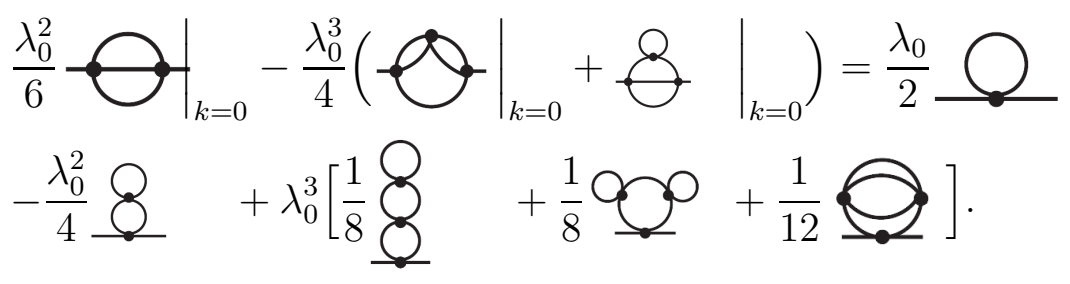

In the right hand side are all tadpole diagrams up to this order. Since the tadpoles do not depend on the external momenta, we can replace this expressios directly in the diagrammatic expression in order to get rid of them. The right hand side diagrams computed at zero external momenta survive. The mass insertions cancel most easily: at one-loop order the condition $\tilde{\Gamma}^{(2)}\left(k=0, \mu_{0}=0, \lambda_{0}\right)=0=\frac{\lambda_{0}}{2} \bigcirc$ implies that the contributions with tadpole insertions appearing in all primitively divergent vertex parts are zero identically up to the perturbative order considered.

Plugging together all these facts into the bare vertex part $\tilde{\Gamma}^{(2)}\left(k, j, \mu_{0}=0, \tilde{\sigma}\right)$ (for further details, the reader is advised to consult Ref. [25]), we end up with

$$
\tilde{\Gamma}^{(2)}\left(k, \mu_{0}=0, \lambda_{0}\right)=k^{2}-\frac{\lambda^{2}}{6}\left[\bigcirc-\bigodot_{k=0}\right]+\frac{\lambda^{3}}{4}\left[\bigcirc_{k=0}\right]
$$

The situation here is completely similar to the massive case, since the number of diagrams are the same, only the renormalization symmetry point is chosen differently due to the infrared divergences occurring in the massless theory. We will keep the same normalization point for the external quasi-momentum modes $i$ and a nonvanishing external momenta.

An arbitrary vertex part including composed operators which is multiplicatively renormalizable in a massless theory is defined by the following expression

$$
\Gamma_{R}^{(L, M)}\left(p_{n}, i_{n}, Q_{n^{\prime}}, j_{n^{\prime}}, g, 0\right)=\left(Z_{\phi}^{(\tau)}\right)^{\frac{M}{2}}\left(Z_{\phi^{2}}^{(\tau)}\right)^{L} \Gamma^{(L, M)}\left(p_{n}, i_{n}, Q_{n^{\prime}}, j_{n^{\prime}}, \lambda_{0}, 0, \Lambda\right)
$$

where the quantities $Z_{\phi}^{(\tau)}, Z_{\phi^{2}}^{(\tau)}$ are the renormalization functions which make the above defined renormalized vertex automatically finite (even when the cutoff $\Lambda$ goes to infinity). As discussed previously our perturbative analysis will utilize only dimensional regularization and forget about the cuttof from now on, since formally the divergences of the integrals manifest themselves as dimensional poles represented by negative powers of the parameter 
$\epsilon=4-d$. In order to prevent confusion, in the above expresssion the identification $\Gamma_{R}^{(2,0} \equiv$ $\tilde{\Gamma}_{R}^{(2)}$ should be taken into account.

The external momentum scale where the theory is renormalized induces a flux in space parameter such that the renormalized vertex parts satisfy the renormalization group equation

$$
\left(\kappa \frac{\partial}{\partial \kappa}+\beta(u) \frac{\partial}{\partial u}-\frac{L}{2} \gamma_{\phi}^{(\tau)}+M \gamma_{\phi^{2}}^{(\tau)}\right) \Gamma_{R}^{(L, M)}\left(p_{n}, i_{n}, Q_{m}, i_{m}^{\prime}, u, 0\right)=0,
$$

where $\beta^{(\tau)}(u)=-\epsilon\left(\frac{\partial \ln u_{0}^{(\tau)}}{\partial u}\right), \gamma_{\phi}^{(\tau)}(u)=\beta^{(\tau)}\left(\frac{\partial \ln Z_{\phi}^{(\tau)}}{\partial u}\right)$ and $\gamma_{\phi^{2}}^{(\tau)}=\beta^{(\tau)}\left(\frac{\partial \ln Z_{\phi^{2}}^{(\tau)}}{\partial u}\right)$. The combinations $\bar{Z}_{\phi^{2}}^{(\tau)}=Z_{\phi^{2}}^{(\tau)} Z_{\phi}^{(\tau)}$ and $\bar{\gamma}_{\phi^{2}}^{(\tau)}=\beta^{(\tau)}\left(\frac{\partial \ln \bar{Z}_{\phi^{2}}^{(\tau)}}{\partial u}\right)$ will be important in what follows. The renormalized theory is defined at fixed $L$. The reader is advised to consult Refs. [2, 22].

We turn our attention to the dimensional crossover regime in the massless theory. We will analyze the one-loop four point contribution and investigate its properties in the limits $L \rightarrow \infty$ and $L \rightarrow 0$. The latter should indicate how small $L$ can be without affecting the validity of the $\epsilon$-expansion.

The graph required involves the combination of two integrals which follow directly from our discussion in the massive case, namely

$$
\begin{aligned}
& I_{2}(k, i, \tilde{\sigma})=\tilde{\sigma} \sum_{l=-\infty}^{\infty} \int \frac{d^{d-1} q}{\left[q^{2}+\tilde{\sigma}^{2} l^{2}\right]\left[(q+k)^{2}+\tilde{\sigma}^{2}(l+i)^{2}\right]}, \\
& \tilde{I}_{2}(k, i, j, \tilde{\sigma})=\tilde{\sigma} \int \frac{d^{d-1} q}{\left[q^{2}+\tilde{\sigma}^{2} i^{2}\right]\left[(q+k)^{2}+\tilde{\sigma}^{2} j^{2}\right]} .
\end{aligned}
$$

We begin with the calculation of the the integral $I_{2}(k, i, \tilde{\sigma})$. Utilize a Feynman parameter $x$. Next integrate over the momentum using Eq. (A2) from Appendix A. We are left with

$$
\begin{aligned}
& I_{2}(k, i, \tilde{\sigma})=\frac{\tilde{\sigma} S_{d-1} \Gamma\left(\frac{d-1}{2}\right) \Gamma\left(2-\frac{(d-1)}{2}\right)}{2} \sum_{l=-\infty}^{\infty} \int_{0}^{1} d x\left[x(1-x)\left(k^{2}+\tilde{\sigma}^{2} i^{2}\right)\right. \\
& \left.\quad+\quad \tilde{\sigma}^{2}(l+i x)^{2}\right]^{\frac{(d-1)}{2}-2} .
\end{aligned}
$$

Next perform the summation using the generalized thermal function as practiced before. Transforming $S_{d-1}$ into $S_{d}$ as previously prescribed, dividing the result by $S_{d}$ and performing the $\epsilon$-expansion of the Gamma functions, we find

$$
I_{2}(k, i, \tilde{\sigma})=\frac{1}{\epsilon}\left(1+\frac{\epsilon}{2}\right)\left[\tilde{\sigma}^{2} i^{2}+k^{2}\right]^{-\frac{\epsilon}{2}}+\frac{\tilde{\sigma}^{-\epsilon}}{2} \int_{0}^{1} d x f_{\frac{1}{2}+\frac{\epsilon}{2}}\left(i x, \sqrt{x(1-x)\left[i^{2}+\frac{k^{2}}{\tilde{\sigma}^{2}}\right]}\right) .(11
$$

In the massless theory, we factor out the external momentum scale. We define $\hat{F}_{\alpha}(k, i ; \tilde{\sigma})=$ $\tilde{\sigma}^{-2 \alpha} \int_{0}^{1} d x f_{\frac{1}{2}+\frac{\epsilon}{2}}\left(i x, \sqrt{x(1-x)\left[i^{2}+\frac{k^{2}}{\tilde{\sigma}^{2}}\right]}\right)$, factorize the external momenta by defining the 
quantity $\hat{r}=\frac{\tilde{\sigma}}{\kappa}$ and identify $I_{2}(\kappa, i, \tilde{\sigma}) \equiv I_{2}(\kappa, i, \hat{r})$ (with similar identification for $\hat{F}_{\alpha}$ ) after this, which is the external momenta scale we need at the symmetry point. By expanding in $\epsilon$ gives the following result:

$$
I_{2}(\kappa, i, \hat{r})=\kappa^{-\epsilon} \frac{1}{\epsilon}\left[1+\frac{\epsilon}{2}-\frac{\epsilon}{2} \ln \left(1+\hat{r}^{2} i^{2}\right)+\frac{\epsilon}{2} \hat{F}_{0}(\kappa, i ; \hat{r})\right] .
$$

Following a similar trend it is a simple task to compute the nondiagonal integral whose solution can be written as

$$
\tilde{I}_{2}(\kappa, i, j, \hat{r})=\frac{\hat{r} \kappa^{-\epsilon}}{2} \int_{0}^{1} d x\left[x(1-x)+\hat{r}^{2}\left(i^{2} x+j^{2}(1-x)\right)\right]^{-\frac{1}{2}} .
$$

In order to get the diagram computed at the symmetry point, we choose the external momentum and quasi-momentum at fixed nonvanishing values. We have already discuss the combinations of last integrals entering in the process of computing the required diagram. After using standard manipulations (see for instance Ref. [31]), implies the outcome

$$
\begin{gathered}
\hat{\zeta}^{(\tau)}(i ; \hat{r})=-\frac{1}{6} \ln \left(1+4 \hat{r}^{2} i^{2}\right)+\frac{1}{6} \hat{F}_{0}(\kappa, 2 i, \hat{r})+\frac{1}{3} \hat{F}_{0}(\kappa, 0, \hat{r})+\frac{\pi \hat{r} \tau}{3} \\
+\frac{2 \hat{r} \tau}{3}(1+2 \tau) \arcsin \left[\frac{1}{\sqrt{1+4 \hat{r}^{2} i^{2}}}\right] .
\end{gathered}
$$

The finite-size contribution function above has the same general structure as that from the massive theory, as it is going to be shown in a moment. Recall that the above coefficient $\tilde{\sigma}$ appears in the definition of the four-point renormalized vertex function. Just like in the massive approach, this factor will multiply the diagrams of this vertex function in arbitrary loop order and can be factored out from our asymptotic analysis.

Before focusing on the asymptotical values for the correction function, it is important to mention that the would be scaling variable in the massless theory $\frac{L}{\xi}$ goes to zero for finite values of $L$ as $\xi \equiv \infty$ in that case. So the cases that could be assessed here are given respectively by the regions $\frac{L}{\xi} \leq 1$. The limit $L \rightarrow \infty$ corresponds to $\frac{L}{\xi} \approx 1$, whereas small values for the distance between the boundary plates are characterized by the limit $\frac{L}{\xi} \rightarrow 0$. In the latter, the question again is how small $L$ can be such that the $\epsilon$-expansion is still valid.

We commence with the large $L$ limit, which is the same as $\hat{r} \rightarrow 0$. Since the linear terms in $\hat{r}$ goes to zero trivially, we restrict ourselves to the evaluation of the terms $\hat{F}_{0}(\kappa, 2 i, \hat{r})$ and 
$\hat{F}_{0}(\kappa, 0, \hat{r})$. Using the definition along with the Boschi-Farina representation in terms of a summation of modified Bessel functions and taking that limit we find

$$
\begin{aligned}
& \hat{F}_{0}(\kappa, 2 i, \hat{r})=4 \sum_{n=1}^{\infty} \int_{0}^{1} d x \cos (4 \pi n i x) K_{0}\left(2 \pi n \sqrt{x(1-x)\left[\hat{r}^{-2}+4 i^{2}\right]}\right) \rightarrow 4 \sum_{n=1}^{\infty} \int_{0}^{1} d x \cos (4 \pi n i x) \\
& \times K_{0}\left(2 \pi n \sqrt{x(1-x)} \hat{r}^{-1}\right)<4 \sum_{n=1}^{\infty} \int_{0}^{1} d x K_{0}\left(2 \pi n \sqrt{x(1-x)} \hat{r}^{-1}\right)
\end{aligned}
$$

where the latter is precisely $\hat{F}_{0}(\kappa, 0, \hat{r})$, and we will show henceforth its convergence in that limit. Using the asymptotic values of the functions for large values of $z$, namely, $K_{0}(z)=\sqrt{\frac{\pi}{2 z}} e^{-z}\left(1+O\left(\frac{1}{z}\right)\right)$, we have

$$
\hat{F}_{0}(\kappa, 0, \hat{r})=2 \hat{r}^{\frac{1}{2}} \sum_{n=1}^{\infty} n^{-\frac{1}{2}} \int_{0}^{1} d x[x(1-x)]^{-\frac{1}{4}} \exp (-n \hat{B}),
$$

where $B=2 \pi \sqrt{x(1-x)} \hat{r}^{-1}$. If we interchange the summation and the integral, we have to compute the summation $\sum_{n=1}^{\infty} n^{-\frac{1}{2}} \exp (-n \hat{B})<\sum_{n=1}^{\infty} \exp (-n \hat{B})=\frac{1}{\exp (\hat{B})-1}=\frac{1}{\hat{B}+\sum_{n=1}^{\infty} \frac{\hat{B}^{n}}{n !}}<$ $\hat{B}^{-1}$. Therefore,

$$
\hat{F}_{0}(\kappa, 0, \hat{r})<\hat{r}^{\frac{3}{2}} \frac{\Gamma\left(\frac{1}{4}\right)^{2}}{\Gamma\left(\frac{1}{2}\right)} \rightarrow 0 .
$$

Last equation is the rigorous proof that the correction goes to zero and one recovers the bulk result.

Let us take the opposite limit $\hat{r} \rightarrow \infty$. We split the several pieces from which $\hat{\zeta}^{(\tau)}(i ; \hat{r})$ is made of and take this limit on each of them. We can easily obtain in that limit the asymptotic behaviors: $-\frac{1}{6} \ln \left(1+4 \hat{r}^{2} i^{2}\right) \rightarrow-\frac{1}{3} \ln (\hat{r} i) ; \frac{2 \hat{r} \tau}{3}(1+2 \tau) \arcsin \left[\frac{1}{\sqrt{1+4 \hat{r}^{2} i^{2}}}\right] \rightarrow \frac{(\tau+2)}{3 i}$. We are left with the nontrivial task of determining the terms proportional to $\hat{F}_{0}(\kappa, 2 i, \hat{r})$ and $\hat{F}_{0}(\kappa, 0, \hat{r})$. Using Eq. (84), it is actually simple to prove that $\frac{1}{3} \hat{F}_{0}(\kappa, 0, \hat{r}) \rightarrow-\frac{2}{3} \ln \hat{r}+\frac{\pi}{3} \hat{r}$.

Performing the identifications $\hat{x}=2 \pi \sqrt{x(1-x)\left[\hat{r}^{-2}+4 i^{2}\right]}$ and $t=\frac{2 i x}{\sqrt{x(1-x)\left[\hat{r}^{-2}+4 i^{2}\right]}}$ into Eq. (81) leads to the following expression

$$
\begin{aligned}
& \hat{F}_{0}(\kappa, 2 i, \hat{r})=2 \gamma+2 \int_{0}^{1} d x \ln \left[\frac{\sqrt{x(1-x)\left[\hat{r}^{-2}+4 i^{2}\right]}}{2}\right]+\int_{0}^{1} d x \frac{1}{\sqrt{x(1-x) \hat{r}^{-2}+4 i^{2} x}} \\
& +\sum_{n=1}^{\infty} \int_{0}^{1} d x\left(\frac{1}{\sqrt{\hat{r}^{-2} x(1-x)+4 i(i-n) x+n^{2}}}-\frac{1}{n}\right) \\
& +\sum_{n=1}^{\infty} \int_{0}^{1} d x\left(\frac{1}{\sqrt{\hat{r}^{-2} x(1-x)+4 i(i+n) x+n^{2}}}-\frac{1}{n}\right) .
\end{aligned}
$$


In this expression we can set $\hat{r}^{-1} \rightarrow 0$. Although the dimensional crossover regime has to do with the behavior of large $\hat{r}$ (small values of $L$ ) we choose to keep the explicit dependence on $i$ for the asymptotic behavior and neglect the other constants. The second and third terms are trivial in this limit, and yield the contributions $2 \ln 2 i$ and $\frac{1}{i}$, respectively. The two last terms involving the infinite summation and the parametric integral are identical to those already worked out in the massive theory. In this limit they produce the quantity $2 \sum_{n=1}^{\infty} i^{2 n} \zeta(2 n+1)$. Defining again the regularized correction for the massless theory $: \hat{\zeta}(i ; \hat{r}):=$ $\hat{\zeta}(i ; \hat{r})-\frac{1}{3} \sum_{n=1}^{\infty} i^{2 n} \zeta(2 n+1)$ which is identical to that for small values of $L$ in the massive theory. Altogether, the regularized finite-size correction for small values of $L(\hat{r} \rightarrow \infty)$ in the massless case reads

$$
: \hat{\zeta}^{(\tau)}(i ; \hat{r}): \rightarrow-\ln \hat{r}+\frac{\pi \hat{r}(1+\tau)}{3}+\frac{(2 \tau+5)}{6 i}
$$

It is simple to see that the finiteness correction is compatible in massive and massless cases. Notice the absence of terms proportional to $\ln i$ which got cancelled along the way in both massless and massive theories. Since the presence of this sort of term in conventional field theory defined for infinite systems implies that all lenght scales are coupled, the absence of them in the mode of external quasi-momentum $i$ shows that there is nothing fundamental on the sector of the finite-size correction which depends on $i$. In other words the layered geometry do not themselves interact strongly up to all length scales, from one plate to the total number of them, since this information is encoded in the logarithm which is absent in the correction. The apperance of the relevant lenght scale $L$ marks a profound difference with the bulk critical behavior even though the expoents are the same. It is a good explanation why surface effects are not important in this regime.

If we choose the Neumann boundary condition $\tau=1$, we find the asymptotic behavior

$$
: \hat{\zeta}^{(\tau)}(i ; \hat{r}): \rightarrow-\ln \hat{r}+\frac{2 \pi \hat{r}}{3}
$$

and the leading divergence on $\hat{r}$ is linear as happens with $P B C$. This is in agreement with the massive case.

On the other hand, if we take Dirichlet boundary condition we have instead

$$
: \hat{\zeta}^{(\tau)}(i ; \hat{r}): \rightarrow-\ln \hat{r}
$$

and the divergence is purely logarithmic as that for $A B C$, which is just compatible with the massive case as well. If $L$ is too small, those singularities take over the poles in $\epsilon$ of 
the one-loop four-point function therefore invalidating the $\epsilon$-expansion results. This is the meaning of the dimensional crossover regime, where finite-size correction effects get bigger than the leading singularities in $\epsilon$ as already discussed for $P B C$ and $A B C$ in Ref. [22].

The reader might have noticed that, except for the $i$-dependent terms appearing in the finite-size correction function in the $L \rightarrow 0$ limit, the rigorous arguments used to determine the correction function for $P B C$ and $A B C$ carries over for $D B C$ and $N B C$. Since we are neglecting the dimensional crossover regime, that is the main reason we refrain from writing another appendix with the derivation of the results for the massless integrals.

With the resources furnished in the present paper in conjumination with those details worked out in Ref. [22] for $A B C$ and $P B C$, the other two-loop diagrams of the four-point function at the symmetry point can be shown to be given by

$$
\begin{aligned}
& { }_{i}^{i} \mathcal{X}_{i}^{i}=3 \tilde{\sigma} \frac{\left(N^{2}+6 N+20\right)}{27} \kappa^{-2 \epsilon}\left\{\frac{1}{\epsilon^{2}}\left(1+\epsilon+2 \epsilon \hat{\zeta}^{(\tau)}(i, \hat{r})\right)\right\}, \\
& \bigcup_{i}^{i}=3 \tilde{\sigma} \frac{(5 N+22)}{27} \kappa^{-2 \epsilon}\left\{\frac{1}{2 \epsilon^{2}}\left(1+\frac{3 \epsilon}{2}+2 \epsilon \hat{\zeta}^{(\tau)}(i, \hat{r})\right)\right\} .
\end{aligned}
$$

Similarly, the derivative of the two- and three-loop diagrams (with respect to $k^{2}$ at the symmetry point) of the two-point vertex part can be computed similarly. From our discussion so far, it is not difficult to find out the following (divergent) expressions

$$
\begin{aligned}
& \frac{\partial}{\partial k^{2}}(\underset{i}{-})_{k^{2}=0}=-\frac{\kappa^{-2 \epsilon}(N+2)}{24 \epsilon}\left[1+\frac{5 \epsilon}{4}-2 \epsilon \hat{W}^{(\tau)}(i, \hat{r})\right], \\
& \frac{\partial}{\partial k^{2}}(\overbrace{i})_{k^{2}=0}=-\frac{\kappa^{-3 \epsilon}(N+2)(N+8)}{162 \epsilon^{2}}\left[1+2 \epsilon-3 \epsilon \hat{W}^{(\tau)}(i ; \hat{r})\right] .
\end{aligned}
$$

Here the finite-size correction for the two-point function as well as the quantities which define it can be written in the form

$$
\begin{aligned}
& \hat{W}^{(\tau)}(i ; \hat{r})=\frac{1}{2} \ln \left[1+\hat{r}^{2} i^{2}\right]+2 \hat{F}^{\prime}{ }_{0}(\kappa, i ; \hat{r})-\hat{\bar{F}}_{0}(\kappa, i ; \hat{r})-3 \hat{r}\left[\hat{H}_{0}(\kappa, i, 0 ; \hat{r})\right. \\
& \left.+\tau \hat{H}_{0}(\kappa, 0, i ; \hat{r})\right]+6 \hat{r}\left[\hat{\mathcal{H}}_{0}(\kappa, i, 0 ; \hat{r})+\tau \hat{\mathcal{H}}_{0}(\kappa, 0, i ; \hat{r})\right], \\
& \hat{F}_{\alpha}(\kappa, i, \hat{r})=\frac{\tilde{\sigma}^{-2 \alpha}}{S_{d}} \int_{0}^{1} f_{\frac{1}{2}+\alpha}\left(i x, \sqrt{x(1-x)\left[\hat{r}^{-2}+i^{2}\right]}\right), \\
& \hat{F}_{\alpha, \beta}(k, i ; \tilde{\sigma})=\frac{\tilde{\sigma}}{S_{d}} \sum_{j=-\infty}^{\infty} \int d^{d-1} q \frac{\hat{F}_{\alpha}(q+k, j+i, \tilde{\sigma})}{\left[q^{2}+\tilde{\sigma}^{2} j^{2}\right]}, \\
& \hat{\bar{F}}_{\alpha}(\kappa, i ; \hat{r})=\tilde{\sigma}^{-2 \alpha} \int_{0}^{1} d x x^{\frac{\alpha}{2}}(1-x) f_{\frac{1}{2}+\alpha}\left(i x, \sqrt{x(1-x)\left[\hat{r}^{-2}+i^{2}\right]}\right), \\
& \hat{H}_{0}(\kappa, i, j ; \hat{r})=\int_{0}^{1} d x(1-x)\left[x(1-x)+\hat{r}^{2}\left(i^{2}(1-x)+j^{2} x\right)^{-\frac{1}{2}}\right],
\end{aligned}
$$


where $\hat{F}^{\prime}{ }_{\alpha}(\kappa, i ; \hat{r})=\left.\frac{\partial \hat{F}_{\alpha, 1}(k, i ; \hat{r})}{\partial k^{2}}\right|_{k^{2}=\kappa^{2}}$.The other amounts appearing in the equation for the correction are defined by

$$
\begin{aligned}
& \hat{\mathcal{H}}_{0}(\kappa, i, j ; \hat{r})=\kappa^{2 \epsilon} \hat{\mathcal{F}}_{0}^{\prime(\tau)}(\kappa, i, j ; \hat{r}) \\
& \hat{\mathcal{F}}_{\alpha, \beta}^{(\tau)}(k, i, j ; \tilde{\sigma}) \equiv \frac{1}{S_{d}} \tilde{\sigma} \int d^{d-1} q \frac{\hat{F}_{\alpha}^{(\tau)}(q+k, j ; \tilde{\sigma})}{\left[q^{2}+\tilde{\sigma}^{2} i^{2}\right]^{\beta}} \\
& \left.\hat{\mathcal{F}}_{\alpha}^{\prime(\tau)}(\kappa, i, j ; \hat{r}) \equiv \frac{\partial \hat{\mathcal{F}}_{\alpha, 1}^{(\tau)}(k, i, j ; \hat{r})}{\partial k^{2}}\right|_{k^{2}=\kappa^{2}} .
\end{aligned}
$$

We have all elements to compute the critical exponents using the massless fields framework. As we are going to show in the next section, all dependence on the finite-size corrections for the several vertex parts disappears in the Wilson functions at the nontrivial attractive infrared fixed point. The main consequence of this is that in the finite-size regime the critical exponents are boundary condition independent.

\section{CRITICAL EXPONENTS IN THE MASSLESS SECTOR}

We are going to be rather brief in the present section. We shall focus solely on the computation of critical exponents using normalization conditions, albeit the results in the present work should be worthwhile in the evaluation of critical exponents using minimal subtraction. The algorithm was already set in the massive case. All we have to do is to replace the massive diagrams by its massless counterpart. It is important to realize that in the perturbative expansion of the primitively bare vertex parts, the bare coupling constant is defined in terms of the bare dimensionless coupling constant through $\lambda_{0}=u_{0} \kappa^{\epsilon}$. This implies that for arbitrary fixed $\kappa$, the perturbative expansion consisting of the multiplication of suitable powers of $\lambda_{0}$ by the diagram eliminate all the $\kappa$ prefactors appearing in each diagram as just discussed in the previous section.

Firstly, use the expansion of $u_{0}^{(\tau)}, Z_{\phi}^{(\tau)}$ and $\bar{Z}_{\phi^{2}}$ in powers of $u$ in exactly the same form as we did before in the massive case. Secondly, employ the analogue of Eq. (95) recalling that now the symmetry point occurs for nonzero momentum and the diagrams correspond to massless fields. We refrain from giving the massless version of Eq. (96), rather we implicitly plug them in the definition of the primitively divergent massless vertex parts. We then obtain the following solution for the renormalization functions (i.e., $u_{0}^{(\tau)}, Z_{\phi}^{(\tau)}$ and $\bar{Z}_{\phi^{2}}$ ) 
coefficients

$$
\begin{aligned}
& a_{1}^{(\tau)}=\frac{(N+8)}{6 \epsilon}\left[1+\frac{\epsilon}{2}+\epsilon \hat{\zeta}^{(\tau)}(i ; \hat{r})\right] \\
& a_{2}^{(\tau)}=\left[\frac{(N+8)}{6 \epsilon}\right]^{2}\left[1+2 \epsilon \hat{\zeta}^{(\tau)}(i ; \hat{r})\right]+\frac{2 N^{2}+23 N+86}{72 \epsilon} \\
& b_{2}^{(\tau)}=-\frac{(N+2)}{144 \epsilon}\left[1+\frac{5 \epsilon}{4}-2 \epsilon \hat{W}^{(\tau)}(i ; \hat{r})\right] \\
& b_{3}^{(\tau)}=-\frac{(N+2)(N+8)}{1296 \epsilon^{2}}\left[1+\frac{5 \epsilon}{4}+3 \hat{\zeta}^{(\tau)}(i ; \hat{r})\right] \\
& c_{1}^{(\tau)}=\frac{(N+2)}{6 \epsilon}\left[1+\frac{\epsilon}{2}+\epsilon \hat{\zeta}^{(\tau)}(i ; \hat{r})\right] \\
& c_{2}^{(\tau)}=\frac{(N+2)(N+5)}{36 \epsilon^{2}}+\frac{2 N^{2}+11 N+14}{72 \epsilon}+\frac{N^{2}+7 N+10}{18 \epsilon} \hat{\zeta}^{(\tau)}(i ; \hat{r}) .
\end{aligned}
$$

We are going to use this information in the calculation of the Wilson functions. We just have to plug these coefficients into Eqs. (101). The nontrivial infrared fixed point value of the coupling constant $u^{*}$ is obtained from the condition $\beta^{(\tau)}\left(u^{*}\right)=0$. It is given by

$$
u^{*}=\left[\frac{6 \epsilon}{N+8}\right]\left\{1+\left[\frac{(9 N+42)}{(N+8)^{2}}-\frac{1}{2}-\hat{\zeta}^{(\tau)}(i ; \hat{r})\right] \epsilon\right\} .
$$

Upon substitution of the fixed point into $\gamma_{\phi}(u)$ one obtains the exponent $\eta$ (the anomalous dimension of the field) up to three-loop order, namely

$$
\eta \equiv \gamma_{\phi}\left(u^{*}\right)=\frac{(N+2)}{2(N+8)^{2}} \epsilon^{2}\left\{1+\epsilon\left[\frac{6(3 N+14)}{(N+8)^{2}}-\frac{1}{4}\right]\right\} \text {. }
$$

Finally, using the relation $\nu^{-1}=2-\bar{\gamma}_{\phi^{2}}^{(\tau)}\left(u^{*}\right)-\eta$, leads us to the result

$$
\nu=\frac{1}{2}+\frac{(N+2)}{4(N+8)} \epsilon+\frac{(N+2)\left(N^{2}+23 N+60\right)}{8(N+8)^{3}} \epsilon^{2} .
$$

In spite of being quite different as far as the $\epsilon$-expansion of the diagrams are concerned, the massless and massive settings yield the same critical exponents as expected from the universality hypothesis. Ultraviolet and infrared analysis are completely equivalent in the evaluation of critical exponents, which reproduce those from the bulk as in $P B C$ and $A B C$ conditions.

What is really appealing in this new formulation inspired in the Nemirovsky and Freed construction for massive fields is that no surface fields are required to implement successfully the finite-size renormalization program for $D B C$ and $P B C$. 


\section{DISCUSSION}

Formerly, there was a certain misunderstanding by some authors concerning the phenomenological finite-size scaling hypothesis. It was thought that the variable $\frac{L}{\xi}$ governed the approach to bulk criticality: whenever $\frac{L}{\xi} \rightarrow \infty$ the $\epsilon$-expansion results shown for the above diagram could be trusted, whereas $\frac{L}{\xi} \leq 1$ would be the regime in which perturbation theory could no longer be reliable. In the modern approach to finite-size criticality proposed in the present work, this scaling variable looses its meaning as discussed in the $A B C$ and $P B C$ cases. In fact, we are going to show next that even in the massive case for $D B C$ and $N B C$, the regions $\frac{L}{\xi} \sim 1$ and $\frac{L}{\xi}<1$ are also available to scrutiny within the present technique.

How small $\frac{L}{\xi}$ (or how large $\tilde{r}$ ) can be without invalidating the $\epsilon$-expansion results? Setting $\mu=\xi^{-1}$, the variable can be rewritten as $\tilde{r}=\frac{\pi \xi}{L}$, so that the limit $L \rightarrow 0$ corresponds to $\tilde{r} \rightarrow \infty$. Before we start our discussion, identify the dimensional single pole $\left(\propto \epsilon^{-1}\right)$ with the $\ln \left(\frac{\Lambda}{\mu}\right) \equiv \ln (\Lambda \xi)$ in a theory regularized with a cutoff, which will be helpful to us in what follows.

The dimensional crossover condition for $N B C$ implies that the linear term in $\tilde{r}$ is greater or equal to that coming from the pole in $\epsilon$. (We can extract further information by identifying the ultraviolet cutoff with the inverse of the lattice constant $a$ as $\Lambda \sim \frac{1}{a}$.) In other words, $L \leq \frac{2 \pi \xi}{3 \ln \left(\frac{\xi}{a}\right)}$

Away from the critical temperature $\xi$ is finite and typically a multiple of the lattice parameter. Take $\xi=10 a$. In that case, $L \leq 9.1 a$. There is a narrow region $9.1 a<L<10 a$ where $\frac{L}{\xi}<1$ and the perturbative expansion is still valid. The number of parallel plates is $n=\frac{L}{a}+1$, where $n$ is an integer. Then, for $n \leq 10.1$ the $\epsilon$-expansion results break down. Since $n$ has to be integer, this condition implies that for $n \leq 11$ the perturbative expansion is invalid. However, it is valid (at least formally) for $n>12$. Hereafter, anytime we mention the region of validity of the $\epsilon$-expansion for $L$ in a certain range, it is implicit that this variation interval on $L$ obeys the criterion $\frac{L}{\xi}<1$. Now take $\xi=100 a$, which implies $L \leq 45.5 a, n \leq 47$. There is a wider region $45.5 a<L<100 a$ which does not invalidate perturbation theory. For $\xi=10^{3} a$, the breaking conditions are $L \leq 303.2 a$ (or $n \leq 305$ ) and the window of validity of perturbation theory is contained in the range $303.2 a<L<10^{3} a$. By allowing $\xi=10^{4} a$ leads to the condition(s) $L \leq 2274 a(, n \leq 2275)$ and a wider range 
$(2274 a<L<10000 a)$ exists which does not invalidate perturbation theory, and so on.

In $P B C$ from Ref. [22], the linear term was equal to $\frac{r}{2}$, where $r=\frac{2 \pi \xi}{L}$. Using exactly the same argument as above in $N B C$, the dimensional crossover condition reads now $L \leq \frac{\pi \xi}{\ln \left(\frac{\xi}{a}\right)}$. When $\xi=10 a$ implies $L \leq 13.64 a$ and $n \leq 15$. For that value of the correlation length, perturbation theory is only valid for $\frac{L}{\xi}>1$. The choice $\xi=100 a$ turns out to produce the breaking conditions $L \leq 68.21 a$ and $n \leq 70$, with a region $68.21 a<L<100 a$ where $\frac{L}{\xi}<1$ does not invalidate $\epsilon$-expansion results. Taking $\xi=10^{3} a$, perturbation theory is invalid for $L \leq 454.8 a$ or $n \leq 456$. The window of validity is $454.8 a<L<1000 a$. Finally, the choice $\xi=10^{4} a$ leads to the dimensional crossover conditions $L \leq 3410.9 a$ and $n \leq 3412$, with a wider region of validity, namely, 3410.9a<L<10000a. Comparing with $N B C$ results, the bulk critical behavior requires a smaller number of plates for $N B C$ than in $P B C$ and a thinner film geometry. Since fewer plates are required in $N B C$ to keep the bulk critical behavior, from the energetic viewpoint it is easier to provoke a bulk-surface transition in $N B C$ than in $P B C$. Therefore, our approach explains why nature chooses $N B C$ as the prototype for the bulk-surface transition, since this information is encoded in the finite-size correction to the bulk behavior.

We turn our attention to the dimensional crossover regime for $D B C$. The logarithmic divergence in $\tilde{r} \rightarrow \infty$ in $D B C$ can be understood similarly: regularizing the theory with a cutoff $\Lambda$ such that $\frac{1}{\epsilon} \sim \ln \left(\frac{\Lambda}{\mu}\right)$. The correction will become as big as the dimensional pole whenever $L \leq \pi a \sim 3.14 a$, which is independent of the bulk correlation length $\xi$. Using the same line of thought for $A B C$ yields $L \leq 2 \pi a \sim 6.28 a$ for the collapse of the bulk critical behavior description in perturbation theory. Both results are independent of the (fixed, finite) bulk correlation length $\xi$. For different fixed bulk correlation lengths one needs $n \leq 5$ $(n \leq 8)$ for $D B C(A B C)$ by demanding integer values for $n$. For $n>6(n>9)$ the bulk critical behavior dominates the finite-size corrections and there is a real experimental possibility of construction of nanodevices for $D B C(A B C)$.

For instance, by slicing a thick material presenting bulk critical behavior in thin films satisfying the above conditions, one could vary the temperature to different values from the bulk critical temperature (variation of the correlation length) and use neutron scattering experiments to see whether the bulk behavior varies for large enough values of $n$. If it does not, the boundary conditions on the limiting plates are either $D B C$ or $A B C$ and we have a practical way to determine the boundary conditions in a certain material. If it does, the 
boundary condition should be either $N B C$ or $P B C$ and the number $n$ should be much bigger than in the previous situation.

The description of the dimensional crossover regime in the massless case is also worthwhile. It is important to mention that finite values of $L$ already implies $\frac{L}{\xi} \rightarrow 0$ since $\xi=\infty$ in the massless (critical temperature) formulation. The important quantity here is the external momenta scale which generates the renormalization flow, although it has no obvious physical interpretation like the connection of the mass with the correlation length. Nevertheless, we can vary it and see what happens with the correction.

For $N B C$, the linear term in the massless case is $\frac{2 \pi \hat{r}}{3}$, where $\hat{r}=\frac{\tilde{\sigma}}{\kappa}\left(\sigma=\frac{\pi}{L}\right)$ as can be verified from Eq. (121). What is left here is to perform the variation of $\kappa$ in units of the inverse multiple of the lattice parameter. Following the same trend we find the critical value $L \leq \frac{2 \pi^{2}}{3 \kappa \ln \left(\frac{1}{\kappa a}\right)}$. For instance, we can set $\kappa^{-1}=10 a, \kappa^{-1}=100 a, \kappa^{-1}=10^{3} a$, and $\kappa^{-1}=10^{4} a$. Obviously, the picture does not change. All the critical values of $L$ below which the system undergoes the dimensional crossover gets a factor of $\pi$ with respect to those from the massive theory. For instance, $\kappa^{-1}=10 a$ implies that $L \leq 28.6(n \leq 30)$; for $\kappa^{-1}=100 a$, one has $L \leq 142.9(n \leq 144)$; when $\kappa^{-1}=10^{3} a$, we obtain $L \leq 952.5(n \leq 954)$; etc.

For $P B C$, a comparison involves the correction to the four-point function which is given by the second, third, fourth and fifth terms from Eq. (61) from Ref. [22] by taking $\tau=0$. Of course, we have to reconstruct that expression (taken at $\kappa=1$ ) by multiplying all terms proportional to $L$ by $\kappa$. Then, the second term gives the logarithmic divergence on $r=\frac{\sigma}{\kappa}$ $\left(\sigma=\frac{2 \pi}{L}\right)$ while the third gives the linear divergence on $r$ and the other terms are not important in what follows. Altogether, the aforementioned correction for small values of $L$ has the linear divergence as the dominant term which invalidates perturbation expansion whenever $L \leq \frac{\pi^{2}}{\kappa \ln \left(\frac{1}{\kappa a}\right)}$. It is not necessary to go into the details, to see that several values of $\kappa$ in the same range as before produce critical values of $L$ which are bigger by a factor of $\pi$ in comparison with the analogous situation in the massive theory. The reader can check that the same feature takes place to $N B C$ when compared to the massless case. This should be not surprising since the fluctuations are enhanced in the massless regime which requires thicker slabs to guarantee the bulk criticality.

Perhaps the most interesting result regards $D B C$ and $A B C$ which have the same critical value for $L$ in both massive and massless regimes. This is so because in the massless and massive regimes the logarithm term for the variables in both situations does not change its 
coefficients. The full unveiling of this invariance remains to be investigated.

In closing this discussion, if these inequalities are not satisfied, the new situation correspond to new critical behavior not belonging to the usual bulk universality class, since the smallness of this variable disturbs the system in an uncontrolable fashion driving it to a "dimensional crossover". Note, however, that the regions $\frac{L}{\xi} \sim 1$ and $\frac{L}{\xi}<1$ are away from the dimensional crossover regime since they are not too close to zero. They can be safely described within the present modern finite-size scaling approach.

\section{CONCLUDING REMARKS}

In this work we have developed a momentum space method to calculate critical exponents for a critical system whose order parameter is defined on a layered geometry with Dirichlet and Neumann boundary conditions imposed on the limiting (parallel) plates at $z=0, L$ with $z$ characterizing the finite-size direction perpendicular to the $(d-1)$-dimensional (hyper)planes of infinite extent along all its linear dimensions. The main features introduced are: $i)$ the tensors needed to construct arbitrary loop diagrams for $1 P I$ vertex parts through the utilization of the exponential representation for the basis eigenfunctions with or without insertion of composite operators which can be renormalized multiplicatively; ii) the representation of the finite-size correction written in terms of a sum of Bessel functions instead of that involving integrals without elementary primitive; iii) massless framework for the computation of critical indices.

There are many similarities with the $P B C$ and $A B C$ treated recently [22]. For instance, the unification of the results for the boundary conditions, except that for $D B C$ this only occurs as long as the external quasi-momentum are set to nonzero values. (For $P B C$ and $A B C$ this unification was obtained using vanishing external quasi-momentum.) This restriction causes no loss of generality, since it is possible to show that $N B C$ can also be formulated with zero external quasi-momenta and the results do not change. We leave this topic for a future work. The region of validity of the $\epsilon$-expansion is consistent either in the massless or in the massive formalism. In that region finite-size scaling is valid, the dominant critical behavior is the one from the bulk with respect to the critical exponents even though the finite-size corrections might appear in other universal quantities, like amplitude ratios [32, 33]. From the dimensional crossover regime viewpoint where the $\epsilon$-expansion results are 
no longer applicable when $L$ gets smaller and smaller, $D B C$ and $A B C$ diverge logarithmically with $L$ whereas $N B C$ and $P B C$ diverge like $L^{-1}$. This confirms a previous claim by Nemirovsky and Freed in their Green's function framework for the massive case.

When the $\epsilon$-expansion ceases to be valid, those finite-size corrections become bigger than the poles in $\epsilon$ (representing logarithmic divergences of the bulk theory). In that case, the correction for $N B C \propto L^{-1}$ in the limit $L \rightarrow 0$ dominates over the term $\ln L$ (which is one piece of the total contribution). In the present framework, the onset of the dimensional crossover is directly connected to surface effects but not in the way previously imagined, i.e., the passage from $(N, d)$ to the $(N, d-1)$ universality class: the interaction between the plates provoke a more complicated effect transliterated in those $L$-dependent terms. In $N B C$ the dimensional crossover regime can be identified with the bulk-surface transition region although the identification is not complete since a proper description of this region should probably include external surface fields as well [12, 34, 35]. In other words, the dimensional crossover regime marks the transmutation of finite-size contributions into surface effects.

From the technical point of view, there are other similarities among $(D B C, N B C)$ and $(A B C, P B C)$. Our utilization of the basis functions in terms of the exponentials for $(D B C$, $N B C)$ makes it possible to use results from $(A B C, P B C)$ analysis like performing summations in the range $(-\infty, \infty)$, since the functional form of these results are invariant in spite of minor modifications in the finite size parameter $r(\tilde{r}, \hat{r}$, etc. $)$. Besides, the terms which have been discarded along the way for being more regular than those kept in all stages of the process revealed themselves identical in their functional form for all of these boundary conditions, with differences obviously in the massless and massive approaches.

But there are also totally different aspects when comparing $(D B C, N B C)$ and $(A B C$, $P B C)$. The latter are boundary conditions whose terms do not break translation invariance. The former actually do have translation invariance breaking terms. Previously, the common belief about translation invariance breaking was necessarily attached to surface contributions. These could stem from two origins, namely, if either surface fields $i$ ) are not allowed to begin with and these contributions are corrections to finite-size effects or ii) are permitted and the subject goes beyond the finite-size problem itself [12, 18]. According to our study here the description of finite-size systems subject to $D B C$ or $N B C$ can be understood entirely out of fields within the volume between the plates without necessity of referring to surface fields. Plus, the breaking of translation invariance in our treatment 
has nothing to do with surface effects, provided the critical system is kept away from the dimensional crossover regime. This is highly desirable in comparison with the deconfined situation: the approach to bulk criticality in the region $0<\frac{L}{\xi} \leq \infty$ clearly leads to a novel modern finite-size scaling paradigm not arising from externally imposed surface fields. The structure unveiled in the present work demonstrates that translation invariance breaking comes from the "nondiagonal terms". These terms actually make it difficult to get rid of mass insertions in order to have a smaller number of diagrams in the computation of critical exponents, for instance. Our prescription to eliminate the mass insertion graphs without invoking external surface fields or any sort of fields other than that representing the confined bulk system might be worthy to take the subject to another level of understanding.

One interesting aspect is to pursue other renormalization schemes like minimal subtraction for massless and massive fields for the boundary conditions just presented. The discussion for massless fields subject to $A B C$ and $P B C$ already appeared in Ref. [22]. A thorough discussion for all boundary conditions utilizing massless and massive fields is still lacking. It would be nice to see whether setting the external quasi-momenta at fixed values should be sufficient to renormalize the massive theory [25].

It is tempting to employ the machinery just developed to treat the case of anisotropic $m$-fold Lifshitz type competing systems [36, 37]. A simple follow-up idea is to take the finite direction perpendicular to the $m$ (-dimensional subspace) competing axes and investigate whether the critical exponents are affected by the introduction of this new ingredient. Amplitude ratios such as that from the susceptibility (see Ref. [38] for the $m=1$ case) and specific heat [39] could also be tackled within the technique introduced here in order to figure out whether the finiteness modify them in comparison with those from the bulk. To extend this topic to its full generality, we could use it in the investigation of finite-size corrections of arbitrary anisotropic competing systems [40,41] and take the finite-size direction perpendicular to all types of competing axes inherent to the problem. The treatment of amplitude ratios for generic competing systems, for instance, those discussed in Refs. [42, 43] poses no obstacle in principle and can be investigated too.

There are many applications in the critical phenomena context which could be unraveled utilizing the results contained in our present results. For instance, further understanding of this topic would lead to the fabrication of new devices involving thin films of materials displaying bulk critical behavior. The importance of our work in guiding this enterprise to 
experimentalists is the indication of how small $L$ can be without spoiling the bulk critical properties, which is certainly important in the nanotechnology scenario.

Since we have shown that the critical exponents for a noncompeting system in a parallel plate geometry with one direction of finite extent is identical of those from the infinite system we could try to find an exact perturbative analytical solution to the uniaxial $(m=1)$ Lifshitz critical exponents. In fact, if we allow the finite-size to be along the competing axis the problematic quartic integral would get transformed into a summation which is easier to perform at least in principle. Taking this achievement for simple enough boundary conditions like $P B C$ would automatically yield the critical exponents of the bulk systems for the uniaxial case. Can this be possible? The results just obtained will help to make sure whether this idea is feasible in the near future.

\section{ACKNOWLEDGMENTS}

MVSS would like to thank CAPES grant number 76640 and CNPq grant number 141912/2012-0 for financial support. JBSJ acknowledges support from CNPq, grant number 142220/2007-8. MML thanks CNPq grant number 232352/2014-3 for partial financial support.

\section{Appendix A: Massive integrals in dimensional regularization}

In the text we have omitted some steps concerning the one-loop integral $I_{2}(k, i, \tilde{r})$ in order to get Eq. (78) due to its similarity with $P B C$ and $A B C$ integrals already worked out in Ref. [22]. We commence by deriving explicitly Eq. (178) along with the nondiagonal integral $\tilde{I}_{2}(k, i, j, \tilde{\sigma}, \mu)$. We shall plug the renormalized mass $\mu$ in all diagrams to be discussed for the reasons discussed in the main text.

Let us get started with Eq. (26a). We factor out the renormalized mass $\mu$ and utilize utilize a Feynman parameter to obtain

$$
\begin{aligned}
& I_{2}^{(\tau)}(k, i ; \tilde{\sigma}, \mu)=\tilde{r} \mu^{-\epsilon} \sum_{j=-\infty}^{\infty} \int_{0}^{1} d x \int d^{d-1} p \\
& \times \frac{1}{\left[p^{2}+2 x k p+x k^{2}+\tilde{r}^{2}\left[(j+i x)^{2}+x(1-x) i^{2}\right]+1\right]^{2}}
\end{aligned}
$$


Dimensional regularization is usually expressed in the Feynman's integrals evaluations through the identity (see Ref.[2])

$$
\int \frac{d^{d} q}{\left(q^{2}+2 k \cdot q+\mu^{2}\right)^{\alpha}}=\frac{1}{2} \frac{\Gamma\left(\frac{d}{2}\right) \Gamma\left(\alpha-\frac{d}{2}\right)\left(\mu^{2}-k^{2}\right)^{\frac{d}{2}-\alpha}}{\Gamma(\alpha)} S_{d}
$$

where $S_{d}$ is the area of the $d$-dimensional unit sphere. Replacing this into the previous expression leads us to

$$
\begin{aligned}
I_{2}^{(\tau)}(k, i ; \tilde{\sigma}, \mu)= & \tilde{r} \mu^{-\epsilon} \frac{1}{2} S_{d-1} \Gamma\left(\frac{d-1}{2}\right) \Gamma\left(2-\frac{(d-1)}{2}\right) \\
& \times \int_{0}^{1} d x \sum_{j=-\infty}^{\infty}\left[x(1-x)\left(k^{2}+i^{2} \tilde{r}^{2}\right)+\tilde{r}^{2}(j+i x)^{2}+1\right]^{\frac{d-1}{2}-2}
\end{aligned}
$$

The summation can be performed using the generalized thermal function identity [24]

$$
\begin{gathered}
D_{\alpha}(a, b)=\sum_{n=-\infty}^{\infty}\left[(n+a)^{2}+b^{2}\right]^{-\alpha} \\
=\frac{\sqrt{\pi}}{\Gamma(\alpha)}\left[\frac{\Gamma\left(\alpha-\frac{1}{2}\right)}{b^{2 \alpha-1}}+f_{\alpha}(a, b)\right]
\end{gathered}
$$

where

$$
f_{\alpha}(a, b)=4 \sum_{m=1}^{\infty} \cos (2 \pi m a)\left(\frac{\pi m}{b}\right)^{\alpha-\frac{1}{2}} K_{\alpha-\frac{1}{2}}(2 \pi m b),
$$

and $K_{\nu}(x)$ is the modified Bessel function of the second kind. By identifying $a(x)=i x$, $b(x)=\tilde{r}^{-1} \sqrt{\left(k^{2}+\tilde{r}^{2} i^{2}\right) x(1-x)+1}$ and performing the continuation $\epsilon=4-d$, we find

$$
\begin{aligned}
I_{2}^{(\tau)}(k, i ; \tilde{\sigma}, \mu)= & \mu^{-\epsilon} \frac{1}{2} S_{d-1} \Gamma\left(\frac{d-1}{2}\right) \sqrt{\pi} \int_{0}^{1} d x \\
& \times\left[\Gamma\left(\frac{\epsilon}{2}\right)\left[x(1-x)\left(k^{2}+i^{2} \tilde{r}^{2}\right)+1\right]^{-\frac{\epsilon}{2}}+f_{\frac{1}{2}+\frac{\epsilon}{2}}(a, b)\right] .
\end{aligned}
$$

Transforming the argument of the $\Gamma$-function with the recipe $\sqrt{\pi} \Gamma\left(\frac{d-1}{2}\right) S_{d-1}=\Gamma\left(\frac{d}{2}\right) S_{d}$ along with the expansion in $\epsilon=4-d$, we get to

$$
\begin{aligned}
I_{2}^{(\tau)}(k, i ; \tilde{\sigma}, \mu)= & S_{d} \mu^{-\epsilon}\left[\frac{1}{\epsilon}\left(1-\frac{\epsilon}{2}\right) \times \int_{0}^{1} d x\left[x(1-x)\left(k^{2}+i^{2} \tilde{r}^{2}\right)+1\right]^{-\frac{\epsilon}{2}}\right. \\
& \left.+\frac{1}{2} \tilde{r}^{-\epsilon} \Gamma\left(2-\frac{\epsilon}{2}\right) \int_{0}^{1} d x f_{\frac{1}{2}+\frac{\epsilon}{2}}\left(i x, \tilde{r}^{-1} \sqrt{x(1-x)\left(k^{2}+i^{2} \tilde{r}^{2}\right)+1}\right)\right]
\end{aligned}
$$

After dividing by $S_{d}$ this object is precisely $I_{2}(k, i ; \tilde{r})$ from Eq. (75) (see also Eq. (76) ). Setting $k=0$ and neglecting $O(\epsilon)$ contributions leads to the following equation

$$
I_{2}^{(\tau)}(k=0, i ; \tilde{r})=\mu^{-\epsilon} \frac{1}{\epsilon}\left[1-\frac{\epsilon}{2}-\frac{\epsilon}{2} \int_{0}^{1} d x \ln \left[x(1-x) i^{2} \tilde{r}^{2}+1\right]+\frac{\epsilon}{2} \tilde{F}_{0}(0, i ; \tilde{r})\right] .
$$


We also need the integral $\tilde{I}_{2}(k, i, j ; \tilde{\sigma}, \mu)$. Factorizing the mass and using a Feynman parameter in this integral from Eq. (26b) one finds

$$
\begin{aligned}
& \tilde{I}_{2}^{(\tau)}(k, i, j ; \tilde{\sigma}, \mu)=\tilde{r} \mu^{-\epsilon} \sum_{j=-\infty}^{\infty} \int_{0}^{1} d x \int d^{d-1} q \\
& \times \frac{1}{\left[q^{2}+2 x k q+x k^{2}+\tilde{r}^{2}\left[\left(i^{2} x+(1-x) j^{2}\right]+1\right]^{2}\right.} .
\end{aligned}
$$

Integrating over $q$ as before, using $\sqrt{\pi} \Gamma\left(\frac{d-1}{2}\right) S_{d-1}=\Gamma\left(\frac{d}{2}\right) S_{d}$ and performing the continuation $\epsilon=4-d$, we can rewrite this object as

$$
\left.\tilde{I}_{2}^{(\tau)}(k, i, j ; \tilde{\sigma}, \mu)=S_{d} \frac{\tilde{r} \mu^{-\epsilon}}{2} \int_{0}^{1} d x\left[x(1-x) k^{2}+\left[x i^{2}+(1-x) j^{2}\right] \tilde{r}^{2}\right)+1\right]^{-\frac{1}{2}},
$$

that results in Eq. (77) after dividing the last expression by $S_{d}$. In particular, the combination which appears to produce the one-loop diagram with all external quasi-momenta set to $i$ is given by

$$
\begin{aligned}
& { }_{i}^{i} 久_{i}^{i}=\tilde{\sigma} \frac{(N+8)}{9}\left[I_{2}(k, 2 i ; \tilde{r})+2 I_{2}(k, 0 ; \tilde{r})+2 \tau \tilde{I}_{2}(k, 0,0 ; \tilde{r})+2 \tau \tilde{I}_{2}(k, 2 i, 0 ; \tilde{r})\right. \\
& \left.+4 \tilde{I}_{2}(k, i, i ; \tilde{r})\right] \text {. }
\end{aligned}
$$

Utilizing the results above mentioned evaluated at $k=0$ yields Eqs. (79). This completes our first task.

Let us consider two- and three-loop integrals. We preclude the dimensional crossover region for too small values of $L$ which spoils the $\epsilon$-expansion analysis.

The easiest two-loop contribution for the four-point vertex function is given by the diagram which consists of the following combination of integrals

$$
\begin{aligned}
& { }_{i}^{i} \mathcal{X}_{i}^{i}=\tilde{\sigma} \frac{\left(N^{2}+6 N+20\right)}{27}\left[I_{2}^{2}(k, 2 i ; \tilde{\sigma}, \mu)+2 I_{2}^{2}(k, 0 ; \tilde{\sigma}, \mu)+4 \tau I_{2}(k, 2 i ; \tilde{\sigma}, \mu)\right. \\
& \times \tilde{I}_{2}(k, 0,2 i ; \tilde{\sigma}, \mu)+4 \tau I_{2}(k, 0 ; \tilde{\sigma}, \mu) \tilde{I}_{2}(k, 0,0 ; \tilde{\sigma}, \mu)+4 \tilde{I}_{2}(k, i, i ; \tilde{\sigma}, \mu)\left[I_{2}(k, 2 i ; \tilde{\sigma}, \mu)\right. \\
& \left.+I_{2}(k, 0 ; \tilde{\sigma}, \mu)\right]+2 \sum_{j=-\infty}^{\infty}\left[\tilde{I}_{2}^{2}(k, j, j+2 i ; \tilde{\sigma}, \mu)+\tilde{I}_{2}^{2}(k, j, j ; \tilde{\sigma}, \mu)+\tau \tilde{I}_{2}(k, j, j+2 i ; \tilde{\sigma}, \mu)\right. \\
& \left.\left.\times \tilde{I}_{2}(k, j+i, j+i ; \tilde{\sigma}, \mu)+\tau \tilde{I}_{2}(k, j, j ; \tilde{\sigma}, \mu) \tilde{I}_{2}(k, j-i, j+i ; \tilde{\sigma}, \mu)\right]\right]
\end{aligned}
$$

computed at zero external momenta. Since the contributions inside the summation in the above expression is regular $\left(O\left(\epsilon^{0}\right)\right)$, we can neglect them. It is then easy to show that

$$
{ }_{i}^{i} 久 \mathcal{X}_{i}^{i}=3 \tilde{\sigma} \frac{\left(N^{2}+6 N+20\right)}{27} \mu^{-2 \epsilon}\left[\frac{1}{\epsilon^{2}}\left(1-\epsilon+2 \epsilon \zeta^{(\tau)}(i ; r)\right)\right] \text {. }
$$


The nontrivial two-loop diagram contributing to the four-point vertex function with arbitrary external momenta was derived in the main text and corresponds to Eq. (59). The integral $\hat{I}_{4}\left(P, k_{3}, i, j, k, \tilde{\sigma}, \mu\right)$ (where $\left.P=k_{1}+k_{2}\right)$ is regular and does not contribute to the singular part of the diagram. At the symmetric point it reads

$$
\begin{aligned}
& -=\frac{(5 N+22)}{27} \tilde{\sigma}\left[I_{4}(0,2 i, i)+2 I_{4}(0,0, i)+3 \tilde{I}_{4}(0, i, i, 0)\right. \\
& \left.+2 \tau \tilde{I}_{4}(0,0,0, i)+\tau \tilde{I}_{4}(0,0,2 i, i)+\tau \tilde{I}_{4}(0,2 i, 0, i)+\tilde{I}_{4}(0, i, i, 2 i)+O\left(\hat{I}_{4}\right)\right]
\end{aligned}
$$

The diagonal terms are composed by the integral $I_{4}(0, i, j)$ which can be written as

$$
I_{4}^{(\tau)}(0, i, j)=\tilde{\sigma} \sum_{j_{1}=-\infty}^{\infty} \int d^{d-1} q \frac{I_{2}^{(\tau)}\left(q, j_{1}-j ; \tilde{\sigma}, \mu\right)}{\left[q^{2}+\tilde{\sigma}^{2}\left(j_{1}-i\right)^{2}+\mu^{2}\right]\left[q^{2}+\tilde{\sigma}^{2} j_{1}^{2}+\mu^{2}\right]} .
$$

When we factorize $\mu$ we find

$$
\begin{aligned}
& I_{4}(0, i, j)=\tilde{r} \mu^{-2 \epsilon} \frac{1}{\epsilon}\left(1-\frac{\epsilon}{2}\right) \int_{0}^{1} d x \sum_{j_{1}=-\infty}^{\infty} \int d^{d-1} q \frac{1}{\left[q^{2}+\tilde{r}^{2}\left(j_{1}-i\right)^{2}+1\right]\left[q^{2}+\tilde{r}^{2} j_{1}^{2}+1\right]} \\
& \times \frac{1}{\left[\left(q^{2}+\tilde{r}^{2}\left(j_{1}-j\right)^{2}\right) x(1-x)+1\right]^{\frac{\epsilon}{2}}}+\tilde{r} \mu^{-2 \epsilon} \frac{1}{2} \tilde{F}_{\frac{\epsilon}{2}, 2}^{(\tau)}(0, i, j) S_{d},
\end{aligned}
$$

where

$$
\tilde{F}_{\frac{\epsilon}{2}, 2}(0, i, j)=\sum_{j_{1}=-\infty}^{\infty} \int d^{d-1} q \frac{\tilde{F}_{\frac{\epsilon}{2}}^{(\tau)}\left(q, j_{1}-j\right)}{\left[q^{2}+\tilde{r}^{2}\left(j_{1}-i\right)^{2}+1\right]\left[q^{2}+\tilde{r}^{2} j_{1}^{2}+1\right]} .
$$

The singular terms in $I_{4}$ come from the high-momentum region of the momentum integrations, since they correspond to ultraviolet divergences. Focusing our attention in the last term, it will contribute to the singular part if and only if the object $\tilde{F}_{\frac{\epsilon}{2}}^{(\tau)}\left(q, j_{1}-j\right)$ is proportional to $q^{p}, p \geq 0$ in the limit $q \rightarrow \infty$. We can discard it in the computation of the singularities if we can prove that it is proportional to $q^{p}$ for $p<0$ in the ultraviolet region. Indeed we can neglect this term as follows.

First, write it in the form

$$
\begin{aligned}
& \left.\tilde{F}_{\frac{\epsilon}{2}}^{(\tau)}(q, j ; \tilde{r})=4 \tilde{r}^{-\epsilon} \int_{0}^{1} d x \sum_{m=1}^{\infty} \cos [2 \pi m j x)\right]\left(\frac{\pi m}{\tilde{r}^{-1} \sqrt{x(1-x)\left(q^{2}+\tilde{r}^{2} j^{2}\right)+1}}\right)^{\frac{\epsilon}{2}} \\
& \quad \times K_{\frac{\epsilon}{2}}\left(2 \pi m \tilde{r}^{-1} \sqrt{x(1-x)\left(q^{2}+\tilde{r}^{2} j^{2}\right)+1}\right) .
\end{aligned}
$$

It follows trivially that

$$
\begin{aligned}
& \tilde{f}_{\frac{\epsilon}{2}}^{\prime}(\tau)(q, j ; \tilde{r})=4 \tilde{r}^{-\epsilon} \int_{0}^{1} d x \sum_{m=1}^{\infty}\left(\frac{\pi m}{\tilde{r}^{-1} \sqrt{x(1-x)\left(q^{2}+\tilde{r}^{2} j^{2}\right)+1}}\right)^{\frac{\epsilon}{2}} \\
& \times K_{\frac{\epsilon}{2}}\left(2 \pi m \tilde{r}^{-1} \sqrt{x(1-x)\left(q^{2}+\tilde{r}^{2} j^{2}\right)+1}\right)>\tilde{F}_{\frac{\epsilon}{2}}^{(\tau)}(q, j ; \tilde{r}) .
\end{aligned}
$$


We analyze the latter henceforth. Since the integrand is symmetric around $x=\frac{1}{2}$ we can write

$$
\begin{aligned}
& \tilde{f}_{\frac{\epsilon}{2}}^{\prime}(\tau)(q, j ; \tilde{r})=8 \tilde{r}^{-\epsilon} \int_{0}^{\frac{1}{2}} d x \sum_{m=1}^{\infty}\left(\frac{\pi m}{\sigma^{-1} \sqrt{x(1-x)\left(q^{2}+\tilde{r}^{2} j^{2}\right)+1}}\right)^{\frac{\epsilon}{2}} \\
& \quad \times K_{\frac{\epsilon}{2}}\left(2 \pi m \tilde{r}^{-1} \sqrt{x(1-x)\left(q^{2}+\tilde{r}^{2} j^{2}\right)+1}\right) .
\end{aligned}
$$

When $q \rightarrow \infty$, we can take a small real parameter $\lambda<<1$ with the property $\lambda q^{2} \rightarrow \infty$. We split the integral into two pieces: in the first one we integrate in the interval $(0, \lambda)$ neglecting the term $x^{2}$ in the integrand, whereas in the interval $\left(\lambda, \frac{1}{2}\right)$ we replace the Bessel function by its asymtoptic form. We then write

$$
\begin{aligned}
& \lim _{q \rightarrow \infty} \tilde{f}_{\frac{\epsilon}{2}}^{\prime}(\tau)(q, j ; \tilde{r})=8 \tilde{r}^{-\epsilon} \sum_{m=1}^{\infty}\left[\int_{0}^{\lambda} d x\left(\frac{\pi m}{\tilde{r}^{-1} \sqrt{x\left(q^{2}+\tilde{r}^{2} j^{2}\right)+1}}\right)^{\frac{\epsilon}{2}}\right. \\
& \times K_{\frac{\epsilon}{2}}\left(2 \pi m \tilde{r}^{-1} \sqrt{x\left(q^{2}+\tilde{r}^{2} j^{2}\right)+1}\right)+\int_{\lambda}^{\frac{1}{2}} d x\left(\frac{\pi m}{\tilde{r}^{-1} \sqrt{x(1-x)\left(q^{2}+\tilde{r}^{2} j^{2}\right)+1}}\right)^{\frac{\epsilon}{2}} \times \\
& \left.\sqrt{\frac{\pi}{4 \pi m \tilde{r}^{-1} \sqrt{x(1-x)\left(q^{2}+\tilde{r}^{2} j^{2}\right)+1}}} \exp \left(-2 \pi m \tilde{r}^{-1} \sqrt{x(1-x)\left(q^{2}+\tilde{r}^{2} j^{2}\right)+1}\right)\right] .
\end{aligned}
$$

The second term goes to zero exponentially and can be disregarded. After replacing the change of variables $y=1+x\left(q^{2}+\tilde{r}^{2} j^{2}\right)$ in the integral, we find

$$
\lim _{q \rightarrow \infty} \tilde{f}_{\frac{\epsilon}{2}}^{\prime}(\tau)(q, j ; \tilde{r})=\frac{8 \tilde{r}^{-\epsilon}}{q^{2}+\tilde{r}^{2} j^{2}} \sum_{m=1}^{\infty}(\pi m)^{\frac{\epsilon}{2}} \int_{1}^{\infty} d y y^{-\frac{\epsilon}{4}} K_{\frac{\epsilon}{2}}\left(2 \pi m \tilde{r}^{-1} \sqrt{y}\right) .
$$

We also know that [31]

$$
\int_{1}^{\infty} d x x^{-\frac{\nu}{2}}(x-1)^{\mu-1} K_{\nu}(a \sqrt{x})=\Gamma(\mu) 2^{\mu} a^{-\mu} K_{\nu-\mu}(a) .
$$

Considering the region outside the dimensional crossover region where $\tilde{r}<\infty$ implies the following asymptotic expression expression

$$
\lim _{q \rightarrow \infty} \tilde{f}_{\frac{\epsilon}{2}}^{\prime}(\tau)(q, j ; \tilde{r})=\frac{8 \tilde{r}^{1-\epsilon}}{q^{2}} \sum_{m=1}^{\infty}(\pi m)^{\frac{\epsilon}{2}-1} K_{\frac{\epsilon}{2}-1}\left(2 \pi m \tilde{r}^{-1}\right) .
$$

Since $\tilde{f}_{\frac{\epsilon}{2}}^{\prime}(\tau)(q, j ; \tilde{r})\left(>\tilde{F}_{\frac{\epsilon}{2}}^{(\tau)}(q, j ; \tilde{r})\right.$ is regular in $\epsilon$, in the ultraviolet region $\tilde{F}_{\frac{\epsilon}{2}}^{(\tau)}(q, j ; \tilde{r})$ and the aforementioned integral involving it are both regular and we do not have to worry about its contribution to the singular part of the integral $I_{4}(0, i, j)$. 
We can write the remaining expression in the form

$$
\begin{aligned}
& I_{4}^{(\tau)}(0, i, j)=\tilde{r} \mu^{-2 \epsilon} \frac{1}{\epsilon}\left(1-\frac{\epsilon}{2}\right) \int_{0}^{1} d x[x(1-x)]^{\frac{-\epsilon}{2}} \sum_{j_{1}=-\infty}^{\infty} \int d^{d-1} q \frac{1}{\left[q^{2}+\tilde{r}^{2}\left(j_{1}-i\right)^{2}+1\right]} \\
& \times \frac{1}{\left[q^{2}+\tilde{r}^{2} j_{1}^{2}+1\right]\left[\left(q^{2}+\tilde{r}^{2}\left(j_{1}-j\right)^{2}\right)+\frac{1}{x(1-x)}\right]^{\frac{\epsilon}{2}}} .
\end{aligned}
$$

We introduce the Feynman parameter $z$ to rewrite last equation as

$$
\begin{aligned}
& I_{4}^{(\tau)}(0, i, j)=\tilde{r} \mu^{-2 \epsilon} \frac{1}{\epsilon}\left(1-\frac{\epsilon}{2}\right) \int_{0}^{1} d x[x(1-x)]^{\frac{-\epsilon}{2}} \sum_{j_{1}=-\infty}^{\infty} \int_{0}^{1} d z \\
& \int \frac{d^{d-1} q}{\left[q^{2}+\tilde{r}^{2} j_{1}^{2}+1+\tilde{r}^{2} i\left(i-2 j_{1}\right) z\right]^{2}\left[\left(q^{2}+\tilde{r}^{2}\left(j_{1}-j\right)^{2}\right)+\frac{1}{x(1-x)}\right]^{\frac{\epsilon}{2}}},
\end{aligned}
$$

followed by another $y$ to melt the two denominators into a single one. We then obtain:

$$
\begin{aligned}
& I_{4}^{(\tau)}(0, i, j)=\tilde{r} \mu^{-2 \epsilon} \frac{\Gamma\left(2+\frac{\epsilon}{2}\right)}{\Gamma\left(\frac{\epsilon}{2}\right) \epsilon}\left(1-\frac{\epsilon}{2}\right) \int_{0}^{1} d x[x(1-x)]^{\frac{-\epsilon}{2}} \sum_{j_{1}=-\infty}^{\infty} \int_{0}^{1} d z \int_{0}^{1} d y y(1-y)^{\frac{\epsilon}{2}-1} \\
& \int \frac{d^{d-1} q}{\left[q^{2}+\tilde{r}^{2} j_{1}^{2}+\tilde{r}^{2}\left[i\left(i-2 j_{1}\right) y z+(1-y) j\left(j-2 j_{1}\right)+y+\frac{1-y}{x(1-x)}\right]^{2+\frac{\epsilon}{2}}\right.} .
\end{aligned}
$$

Employing Eq. (A2) to perform the momentum integration as well as our recipe to transform the unit sphere area $S_{d-1}$ into $S_{d}$, one can show that

$$
\begin{aligned}
& I_{4}^{(\tau)}(0, i, j)=\frac{\tilde{r} \mu^{-2 \epsilon} S_{d}}{2 \sqrt{\pi}} \frac{\Gamma\left(2-\frac{\epsilon}{2}\right) \Gamma\left(\frac{1}{2}+\epsilon\right)}{\Gamma\left(\frac{\epsilon}{2}\right) \epsilon}\left(1-\frac{\epsilon}{2}\right) \int_{0}^{1} d x[x(1-x)]^{\frac{-\epsilon}{2}} \int_{0}^{1} d y y(1-y)^{\frac{\epsilon}{2}-1} \\
& \sum_{j_{1}=-\infty}^{\infty}\left[\tilde{r}^{2}\left[j_{1}^{2}+i^{2} y z+(1-y) j^{2}-2 j_{1}(i y z+(1-y) j)\right]+y+\frac{1-y}{x(1-x)}\right]^{-\frac{1}{2}-\epsilon} .
\end{aligned}
$$

By evaluating the summation using the generalized thermal function upon the identifications $a=-i y z-(1-y) j, b=\sqrt{i^{2} y z(1-y z)+j^{2} y(1-y)+\tilde{r}^{-2}\left(y+\frac{1-y}{x(1-x)}\right)}$ and absorbing the factor $S_{d}$, that integral reads

$$
\begin{aligned}
& I_{4}^{(\tau)}(0, i, j)=\frac{\tilde{r}^{-2 \epsilon} \mu^{-2 \epsilon}}{2 \epsilon}\left(1-\frac{\epsilon}{2}\right) \frac{\Gamma\left(2-\frac{\epsilon}{2}\right)}{\Gamma\left(\frac{\epsilon}{2}\right)} \int_{0}^{1} d x[x(1-x)]^{\frac{-\epsilon}{2}} \int_{0}^{1} d y y(1-y)^{\frac{\epsilon}{2}-1} \\
& \times\left[\Gamma(\epsilon)\left(i^{2} y z(1-y z)+j^{2} y(1-y)+\tilde{r}^{-2}\left(y+\frac{1-y}{x(1-x)}\right)\right)^{-\epsilon}\right. \\
& \left.+f_{\frac{1}{2}+\epsilon}\left(-i y z-(1-y) j, \sqrt{i^{2} y z(1-y z)+j^{2} y(1-y)+\tilde{r}^{-2}\left(y+\frac{1-y}{x(1-x)}\right)}\right)\right]
\end{aligned}
$$

The integral over $y$ presents a pole in $y=1$. We then evaluate it at $y=1$ [2], which is not only a lot easier but maintains the essential pole contribution of interest. Expanding in $\epsilon$ 
and getting rid of $O\left(\epsilon^{0}\right)$ terms, we finally obtain

$$
I_{4}^{(\tau)}(0, i, j)=\mu^{-2 \epsilon} \frac{1}{2 \epsilon^{2}}\left(1-\frac{\epsilon}{2}-\epsilon \int_{0}^{1} d z \ln \left[\tilde{r}^{2} i^{2} z(1-z)+1\right]+\epsilon \tilde{F}_{0}(0, i ; \tilde{r})\right) .
$$

We still have to compute the nondiagonal contribution coming from $\tilde{I}_{4}(0, i, j, l)$. It can be expressed in terms of the one-loop integral $I_{2}(k, i, \tilde{\sigma}, \mu)$ as

$$
\tilde{I}_{4}(0, i, j, l)=\tilde{\sigma} \int d^{d-1} q \frac{I_{2}^{(\tau)}(q, l, \tilde{\sigma}, \mu)}{\left[q^{2}+\tilde{\sigma}^{2} j^{2}+\mu^{2}\right]\left[q^{2}+\tilde{\sigma}^{2} i^{2}+\mu^{2}\right]} .
$$

Extracting the mass from the integrand we get to

$$
\tilde{I}_{4}(0, i, j, l)=\tilde{r} \mu^{-2 \epsilon} \int d^{d-1} q \frac{I_{2}^{(\tau)}(q, l ; \tilde{r})}{\left[q^{2}+\tilde{r}^{2} j^{2}+1\right]\left[q^{2}+\tilde{r}^{2} i^{2}+1\right]} .
$$

Note that the integrand in the last expression is symmetric by the change $i \rightarrow j$. This integral differs from $I_{4}(0, i, j)$ because $\left.i\right)$ the summation over $j_{1}$ is missing, $\left.i i\right)$ in the oneloop subdiagram there appears only a external quasi-momentum $l$, iii) each propagator in the "external bubble" are attached to external quasi-momentum. The computation is entirely analogous with the above computation for $I_{4}(0, i, j)$. Picking out only the singular term and putting aside the regular terms we are led to

$$
\tilde{I}_{4}(0, i, j, l)=\frac{\tilde{r} \mu^{-2 \epsilon}}{2 \epsilon} \int_{0}^{1} d x\left[\tilde{r}^{2}\left(i^{2} x+(1-x) j^{2}\right)+1\right]^{-\frac{1}{2}} .
$$

Now, all we have to do is to take into account the combinations of the integrals just computed. The nontrivial two-loop diagram of the four-point function has the following result:

$$
\overbrace{i}^{i} \bigcup_{i}^{i}=3 \tilde{\sigma} \frac{(5 N+22)}{27} \mu^{-2 \epsilon}\left\{\frac{1}{2 \epsilon^{2}}\left(1-\frac{\epsilon}{2}+2 \epsilon \zeta^{(\tau)}(i, \tilde{r})\right)\right\} \text {. }
$$

The two-point vertex part two-loop diagram which interests us is

$$
\bigodot_{i}=\left(\frac{N+2}{3}\right)\left[I_{3}(k, i, \tilde{\sigma}, \mu)+3 \tilde{I}_{3}(k, i, 0, \tilde{\sigma}, \mu)+3 \tau \tilde{I}_{3}(k, 0, i, \tilde{\sigma}, \mu)\right] \text {. }
$$

We actually need the derivative of this diagram computed at zero external momenta. Let us first consider $I_{3}((k, i, \tilde{\sigma}, \mu)$. Factorizing the mass, it can be written as follows

$$
I_{3}(k, i, \tilde{\sigma}, \mu)=\tilde{r} \mu^{2-\epsilon} \sum_{j_{1}=-\infty}^{\infty} \int \frac{d^{d-1} q_{1} I_{2}\left(q_{1}+k, j_{1}+i ; \tilde{r}\right)}{\left(q_{1}^{2}+\tilde{r}^{2} j_{1}^{2}+1\right)} .
$$

The solution of the subdiagram is then substituted into this expression and yields

$$
\begin{aligned}
& I_{3}(k, i, \tilde{\sigma}, \mu)=\mu^{2-2 \epsilon} \frac{1}{\epsilon}\left\{\left(1-\frac{\epsilon}{2}\right) \tilde{r} \int_{0}^{1} d x \sum_{j_{1}=-\infty}^{\infty} \int \frac{d^{d-1} q_{1}}{\left[q_{1}^{2}+\tilde{r}^{2} j_{1}^{2}+1\right]\left[\left(q_{1}=k\right)^{2}++\tilde{r}^{2}\left(j_{1}+i\right)^{2}+1\right]}\right. \\
& \left.+\frac{\epsilon}{2} \tilde{F}_{\frac{\epsilon}{2}, 1}^{(\tau)}\left(q_{1}+k, j_{1}+i ; \tilde{r}\right)\right\},
\end{aligned}
$$


where $\tilde{F}_{\frac{\epsilon}{2}, 1}^{(\tau)}\left(q_{1}+k, j_{1}+i ; \tilde{r}\right)$ was defined in Eq. (90a). The remaining of the computation is very similar to what was worked out for $P B C$ and $A B C$; the reader is advised to consult Ref. ([22]) for further details. The derivative at zero external momentum can be shown to be given by

$$
\left.\frac{\partial I_{3}(k, i ; \tilde{r})}{\partial k^{2}}\right|_{k^{2}=0}=-\frac{\mu^{-2 \epsilon}}{8 \epsilon}\left[1-\frac{\epsilon}{4}+\epsilon \tilde{W}_{0}(i ; \tilde{r})\right]
$$

where $\tilde{W}_{0}(i ; \tilde{r})=\tilde{G}_{0}(i ; \tilde{r})+\tilde{H}_{0}(i ; \tilde{r})-4 \tilde{F}_{0}^{\prime}(\tau)(i ; \tilde{r})$,

$$
\begin{aligned}
& \tilde{G}_{0}(i ; \tilde{r})=-\frac{1}{2}-2 \int_{0}^{1} \int_{0}^{1} d x d y(1-y) \ln \left[y(1-y) i^{2}+(1-y) \tilde{r}^{-2}+\frac{y \tilde{r}^{-2}}{x(1-x)}\right],( \\
& \tilde{H}_{0}(i ; r)=2 \int_{0}^{1} \int_{0}^{1} d x d y(1-y) f_{\frac{1}{2}}\left(i y, \sqrt{\left.y(1-y) i^{2}+(1-y) \tilde{r}^{-2}+\frac{y \tilde{r}^{-2}}{x(1-x)}\right)},\right.
\end{aligned}
$$

with $\tilde{F}_{0}^{\prime(\tau)}(i ; \tilde{r})$ defined in Eq. (包). We have to compute $\left.\frac{\partial}{\partial k^{2}} \tilde{I}_{3}(k, i, j ; \tilde{\sigma}, \mu)\right|_{k^{2}=0}$. First, we scale out the mass to write it as

$$
\tilde{I}_{3}(k, i, j ; \tilde{\sigma}, \mu)=\tilde{r} \mu^{2-\epsilon} \int \frac{d^{d-1} q I_{2}(q+k, i ; \tilde{r})}{\left(q^{2}+\tilde{r}^{2} j^{2}+1\right)}
$$

From the solution of the subdiagram we have the following intermediate result

$$
\begin{aligned}
& \tilde{I}_{3}(k, i, j ; \tilde{r})=\frac{\mu^{2-2 \epsilon}}{\epsilon}\left[\tilde{r}\left(1-\frac{\epsilon}{2}\right) \int_{0}^{1} d x[x(1-x)]^{-\frac{\epsilon}{2}} \int \frac{d^{d-1} q I_{2}(q+k, i ; \tilde{r})}{\left(q^{2}+\tilde{r}^{2} j^{2}+1\right)\left[(q+k)^{2}+\tilde{r}^{2} i^{2}+1\right]^{\frac{\epsilon}{2}}}\right. \\
& \left.+\frac{\epsilon}{2} \tilde{r} \int \frac{d^{d-1} q \tilde{F}_{\frac{\epsilon}{2}}^{(\tau)}(k, i ; \tilde{r})}{\left(q^{2}+\tilde{r}^{2} j^{2}+1\right)}\right] .
\end{aligned}
$$

Let us define the integral

$$
\tilde{i}_{3}=\int \frac{d^{d-1} q I_{2}(q+k, i ; \tilde{r})}{\left(q^{2}+\tilde{r}^{2} j^{2}+1\right)\left[(q+k)^{2}+\tilde{r}^{2} i^{2}+1\right]^{\frac{\epsilon}{2}}} .
$$

Introduce a parameter $y$ in order to write it in the form

$$
\tilde{i}_{3}=\int_{0}^{1} \int \frac{\Gamma\left(1+\frac{\epsilon}{2}\right) d^{d-1} q d y y^{\frac{\epsilon}{2}-1}}{\Gamma\left(\frac{\epsilon}{2}\right)\left[q^{2}+2 k q y+k^{2} y+\tilde{r}^{2}\left(j^{2}(1-y)+i^{2} y\right)+1-y+\frac{y}{x(1-x)}\right]^{1+\frac{\epsilon}{2}}} .
$$

Next integrate over $q$ and transform $S_{d-1}$ into $S_{d}$ using the prescription already stated. When we use the definition Eq. (90c) together with our labor on $\tilde{i}_{3}$ leads us to conclude that

$$
\begin{aligned}
& \tilde{I}_{3}(k, i, j ; \tilde{r})=\frac{\mu^{2-2 \epsilon}}{\epsilon}\left[\frac{\tilde{r} \Gamma\left(2-\frac{\epsilon}{2}\right) \Gamma\left(-\frac{1}{2}+\epsilon\right)}{\sqrt{\pi} \Gamma\left(\frac{\epsilon}{2}\right)}\left(1-\frac{\epsilon}{2}\right) \int_{0}^{1} d x[x(1-x)]^{-\frac{\epsilon}{2}} \int d y y^{\frac{\epsilon}{2}-1}\right. \\
& \left.\left(k^{2} y(1-y)+\tilde{r}^{2}\left(j^{2}(1-y)+i^{2} y\right)+1-y+\frac{y}{x(1-x)}\right)^{\frac{1}{2}-\epsilon}+\frac{\epsilon}{2} \mathcal{F}_{\frac{\epsilon}{2}, 1}^{(\tau)}(k, i, j ; \tilde{r})\right] .
\end{aligned}
$$


Taking the derivative at zero external momentum, performing the expansion in $\epsilon$, discarding $O(\epsilon)$ contributions and using the definition Eq. (90d) we get the desired expression, namely

$$
\begin{aligned}
& \left.\frac{\partial \tilde{I}_{3}(k, i, j ; r)}{\partial k^{2}}\right|_{k^{2}=0}=-\mu^{-2 \epsilon}\left[\frac{\tilde{r}}{4} \int_{0}^{1} d x \int_{0}^{1} d y(1-y)\left(\tilde{r}^{2}\left(j^{2}(1-y)+i^{2} y\right)+1-y+\frac{y}{x(1-x)}\right)^{-\frac{1}{2}}\right. \\
& \left.-\frac{1}{2} \mathcal{F}_{0}^{\prime(\tau)}(i, j ; \tilde{r})\right]
\end{aligned}
$$

Plugging the previous expression in the combinations appearing in the two-loop graph, we get to an expression which goes beyond the result from the simple $P B C$ and $A B C$, or in other words

$$
\begin{aligned}
& \left.\frac{\partial}{\partial k^{2}}\left(\underset{i}{ } \mathcal{O}_{i}\right)\right|_{k^{2}=0}=-\frac{\mu^{-2 \epsilon}(N+2)}{24 \epsilon}\left[1-\frac{\epsilon}{4}+\epsilon \tilde{W}^{(\tau)}(i, \tilde{r})\right], \\
& \tilde{W}(i, \tilde{r})=\tilde{W}_{0}(i ; \tilde{r})+6 \tilde{r}\left[\int_{0}^{1} d x \int_{0}^{1} d y(1-y)\left(\tilde{r}^{2} i^{2} y+1-y+\frac{y}{x(1-x)}\right)^{-\frac{1}{2}}+\tau \int_{0}^{1} d x \int_{0}^{1} d y\right. \\
& \left.\left.\times(1-y)\left(\tilde{r}^{2} i^{2}(1-y)+1-y+\frac{y}{x(1-x)}\right)^{-\frac{1}{2}}\right]-12\left[\mathcal{F}_{0}^{(\tau)}(i, 0 ; \tilde{r})+\tau \mathcal{F}_{0}^{(\tau)}(0, i ; \tilde{r})\right] . \quad \text { (A } 46 \mathrm{~b}\right)
\end{aligned}
$$

The three-loop diagram for the two-point function has a similar systematics. We just need to compute a combination of the objects $\left.\frac{\partial I_{5}(k, i, \tilde{\sigma}, \mu)}{\partial k^{2}}\right|_{k^{2}=0},\left.\frac{\partial \tilde{I}_{5}(k, i, 0, \tilde{\sigma}, \mu)}{\partial k^{2}}\right|_{k^{2}=0}$ and $\left.\frac{\partial \tilde{I}_{5}(k, 0, i, \tilde{\sigma}, \mu)}{\partial k^{2}}\right|_{k^{2}=0}$. The first one can be checked to be given by the following expression:

$$
\left.\frac{\partial I_{5}(k, i, \tilde{\sigma}, \mu)}{\partial k^{2}}\right|_{k^{2}=0}=\mu^{-3 \epsilon}\left(-\frac{1}{6 \epsilon^{2}}\right)\left[1-\frac{\epsilon}{4}+\frac{3 W_{0}^{(\tau)}(i, \tilde{r})}{2}\right],
$$

where $W_{0}^{(\tau)}=-\frac{1}{2}-2 \int_{0}^{1} \int_{0}^{1} d x d y(1-y) \ln \left[y(1-y) i^{2}+(1-y) \tilde{r}^{-2}+\frac{y \tilde{r}^{-2}}{x(1-x)}\right]+2 \int_{0}^{1} \int_{0}^{1} d x d y(1-$ y) $f_{\frac{1}{2}}\left(i y, \sqrt{y(1-y) i^{2}+(1-y) \tilde{r}^{-2}+\frac{y \tilde{r}^{-2}}{x(1-x)}}\right)-4 \tilde{F}_{0}^{\prime}(i ; \tilde{r})$. This is precisely equal to the contribution from $P B C$ in Ref. [22]. We shall discuss the computation of the nondiagonal contribution. First, notice that $\tilde{I}_{5}(k, i, j, \tilde{\sigma}, \mu)$ can be written in the form

$$
\tilde{I}_{5}(k, i, j, \tilde{\sigma}, \mu)=\mu^{2-3 \epsilon} \tilde{r} \int \frac{d^{d-1} q I_{2}^{2}(q+k, j, \tilde{r})}{q^{2}+\tilde{r}^{2} i^{2}+1} .
$$

Replacing the value of the subdiagram already computed previously (with $S_{d}$ already divided), we find

$$
\begin{aligned}
& \tilde{I}_{5}(k, i, j, \tilde{\sigma}, \mu)=\mu^{2-3 \epsilon} \frac{1}{\epsilon^{2}}\left\{\tilde{r} \int_{0}^{1} d x \int \frac{d^{d-1} q}{\left[q^{2}+\tilde{r}^{2} i^{2}+1\right]\left[(q+k)^{2}+\tilde{r}^{2} j^{2}+\frac{1}{x(1-x)}\right]^{\epsilon}}\right. \\
& \left.+\tilde{r} \epsilon \int \frac{d^{d-1} q F_{\frac{\epsilon}{2}}(q+k, j, \tilde{r})}{q^{2}+\tilde{r}^{2} i^{2}+1}\right\} .
\end{aligned}
$$


Now utilize another Feynman parameter, integrate over $q$ (transform the product $S_{d-1} \Gamma\left(\frac{d-1}{2}\right)$ into $S_{d} \Gamma\left(\frac{d}{2}\right)$ as described in the text) and divide the result by $S_{d}$. Expand the remaining $\Gamma$ functions in $\epsilon$ and neglect $O\left(\epsilon^{0}\right)$. Next, taking the derivative at zero external momenta we find

$$
\begin{aligned}
& \left.\frac{\partial \tilde{I}_{5}(k, i, j, \tilde{\sigma}, \mu)}{\partial k^{2}}\right|_{k^{2}=0}=\mu^{-3 \epsilon}\left(-\frac{1}{2 \epsilon}\right)\left\{\tilde { r } \int _ { 0 } ^ { 1 } d y ( 1 - y ) \int _ { 0 } ^ { 1 } d x \left[\tilde{r}^{2}\left(i^{2}(1-y)+j^{2} y\right)+1-y\right.\right. \\
& \left.\left.+\frac{y}{x(1-x)}\right]^{-\frac{1}{2}}-2 \mathcal{F}_{0}^{\prime(\tau)}(i, j, \tilde{r})\right\}
\end{aligned}
$$

where $\mathcal{F}_{0}^{(\tau)}(i, j, \tilde{r})$ was defined in Eq. (90d). The other terms involve the integration of products of the type $I_{2} \tilde{I}_{2}$ and $\tilde{I}_{2} \tilde{I}_{2}$. The former can be shown to be $O\left(\epsilon^{0}\right)$ whereas the former are $O(\epsilon)$. Both terms which contribute a total of ten integrals in the computation of the three-loop graph of the two-point function are all regular and can be neglected in the evaluation of the singular part of the diagram.

With the resources furnished in this Appendix, the reader is invited to check that the following expression holds

$$
\frac{\partial}{\partial k^{2}}\left(\mathcal{i}_{i}\right)_{k^{2}=0}=-\frac{\mu^{-3 \epsilon}(N+2)(N+8)}{162 \epsilon^{2}}\left[1-\frac{\epsilon}{4}+\frac{3 \epsilon}{2} \tilde{W}^{(\tau)}(i ; \tilde{r})\right] .
$$


[1] K. G. Wilson, Phys. Rev. B 4, 3174 (1971); 4, 3184 (1971); K. G. Wilson and M. E. Fisher, Phys. Rev. Lett. 28, 240 (1972); K. G. Wilson, Phys. Rev. Lett. 28, 548 (1972).

[2] D. J. Amit, and V. Martin-Mayor, Field Theory, the Renormalization Group and Critical Phenomena, 3rd ed. (World Scientific, Singapore, 2005).

[3] H. B. Casimir, Proc. K. Ned. Akad. Wet. 51, 793 (1948).

[4] Z. Borjan and P. J. Upton, Phys. Rev. Lett. 101, 125702 (2008).

[5] A. Gambassi, J. Phys.: Conf. Series 161, 012037 (2009).

[6] C. Farina, Braz. J. Phys. 36, 1137 (2006).

[7] R. Garcia and M. H. W. Chan, Phys. Rev. Lett. 83, 1187 (1999); A. Ganshin, S. Scheidemantel, R. Garcia, and M. H. W. Chan, Phys. Rev. Lett. 97, 075301 (2006).

[8] J. Goyon, A. Colin, G. Ovariez, A. Ajdari, and L. Bocquet, Nature 454, 84 (2008); J. Goyon, A. Colin, and L. Bocquet, Soft Matter 6, 2668 (2010).

[9] T. P. Chen and F. M. Gasparini, Phys. Rev. Lett. 40, 331 (1978); F. M. Gasparini, G. Agnolet, and J. D. Reppy, Phys. Rev. B 29, 138 (1984); F. M. Gasparini, M. O. Kimball, K. P. Mooney, and M. Diaz-Avila, Rev. Mod. Phys. 80, 1009 (2008).

[10] B. A. Scheibner, M. R. Meadows, R. C. Mockler, and W. J. O'Sullivan, Phys. Rev. Lett. 43, 590 (1979); M. R. Meadows, B. A. Scheibner, R. C. Mockler, and W. J. O'Sullivan, Phys. Rev. Lett. 43, 592 (1979).

[11] R. Höhmann, U. Kuhl, H. J. Stöckmann, J. D. Urbina, M. R. Dennis, Phys. Rev. E 79, 016203 (2009).

[12] H. W. Diehl, in Phase Transitions and Critical Phenomena, edited by C. Domb and J. L. Lebowitz, (Academic, London, 1986), Vol. 10, p. 76.

[13] S. Sandfeld, Z. Budrikis, S. Zapperi, D. F. Castellanos, J. Stat. Mech. 2, 02011 (2015).

[14] X. Zhou and Z. Zhang, Int. J. Mol. Sci. 14(12), 24135 (2013).

[15] H. Chamati, J. Phys. A: Math. Theor. 41375002 (2008).

[16] M. E. Fisher, in Critical Phenomena, Proceedings of the 1970 Enrico Fermi International School of Physics, Course No. 51, edited by M. S. Green (Academic, New York, 1971), p. 1.

[17] M. E. Fisher, Rev. Mod. Phys. 46, 597 (1974); M. E. Fisher and M. N. Barber, Phys. Rev. Lett. 28, 1516 (1972). 
[18] M. N. Barber, in Phase Transitions and Critical Phenomena, edited by C. Domb and J. L. Lebowitz, (Academic, New York, 1983), Vol. 8, p. 145.

[19] V. Privman in Finite Size Scaling and Numerical Simulations in Statistical Mechanics, edited by V. Privman (World Scientific, Singapore, 1990), p. 1.

[20] V. Privman and M. E. Fisher, Phys. Rev. B 30, 322 (1984).

[21] A. M. Nemirovsky and K. F. Freed, J. Phys. A: Math Gen. 18, L319 (1985); A. M. Nemirovsky and K. F. Freed, Nucl. Phys. B 270, [FS16], 423 (1986).

[22] J. B. da Silva, Jr. and M. M. Leite, J. Math. Phys. 53, 043303 (2012).

[23] N. F. Svaiter, J. Math. Phys. 45, 4524 (2012).

[24] H. Boschi-Filho and C. Farina, Phys. Lett. A 205, 255 (1995).

[25] P. R. S. Carvalho and M. M. Leite, J. Math. Phys. 54, 093301 (2013).

[26] M. Krech and S. Dietrich, Phys. Rev. Lett. 66, 345 (1991); 67, 1055 (1991); Phys. Rev. A 46, 1886 (1992); Phys. Rev. A 46, 1922 (1992).

[27] E. Brezin, J. C. Le Guillou and J. Zinn-Justin, Phys. Rev. D 8, 434 (1973).

[28] E. Brezin, J. C. Le Guillou and J. Zinn-Justin, in Phase Transitions and Critical Phenomena, edited by C. Domb and M. S. Green, (Academic Press, London, 1976), Vol. 6, p. 127.

[29] A. A. Vladimirov, D. I. Kazakov and O. V. Tarasov, Sov. Phys. JETP 50(3), 521 (1979).

[30] J. Naud, I. Nemenmann, M. Van Raamsdonk and V. Periwal, Nucl. Phys. B 540, 533 (1999).

[31] I. S. Gradshteyn and I. M. Ryzhik, Table of Integrals Series and Products, (Academic Press, New York, 2000).

[32] M. M. Leite, A. M. Nemirovsky, and M. D. Coutinho-Filho, J. Magn. Magn. Mater. 104-107, $181(1992)$.

[33] M. M. Leite, M. Sardelich, and M. D. Coutinho-Filho, Phys. Rev. E 59, 2683 (1999).

[34] C. D. Fosco and N. F. Svaiter, J. Math. Phys. 42, 5185 (2001).

[35] M. I. Caicedo and N. F. Svaiter, J. Math. Phys. 45, 179 (2004).

[36] M. M. Leite, Phys. Rev. B 67, 104415 (2003).

[37] P. R. S. Carvalho and M. M. Leite, Ann. Phys. 324, 178 (2009).

[38] M. M. Leite, Phys. Rev. B 61, 14691 (2000).

[39] M. M. Leite, Phys. Rev. B 68, 052408 (2003).

[40] M. M. Leite, Phys. Lett. A 326, 281 (2004); Phys. Rev. B 72, 224432 (2005).

[41] P. R. S. Carvalho and M. M. Leite, Ann. Phys. 325, 151 (2010). 
[42] C. F. Farias and M. M. Leite, J. Stat. Phys. 148, 972 (2012).

[43] M. I. Sena Jr. and M. M. Leite, J. Phys. Conf. Series 574, 012170 (2015). 